\title{
3 Mediennutzung und Schulleistung
}

Ein erster Themenschwerpunkt des Berliner Längsschnitts Medien war die genauere Betrachtung des Wirkzusammenhangs schulischer Leistungen und häuslichen Medienkonsums. Schulische Leistungen sollen mit problematischer Mediennutzung im Sinne einer zeitlich exzessiven oder einer inhaltlich problematischen Mediennutzung in Beziehung gesetzt werden. Dabei geht es darum, den Stellenwert und den ursächlichen Einfluss der Mediennutzungsmuster im multifaktoriellen Beziehungsgeflecht verschiedener Erklärungsfaktoren (neben Variablen der Persönlichkeit und des sozialen Umfelds) zu ergründen.

Zunächst wird der allgemeine Forschungsstand zum Thema Mediennutzung und Schulleistung zusammengefasst (Kapitel 3.1), dann die Ergebnisse des Berliner Längsschnitts Medien präsentiert (Kapitel 3.2) und mit Ersterem in Beziehung gesetzt (Kapitel 3.3).

\subsection{Theoretische Grundlagen und Literaturüberblick - Mediennutzung und Schulleistung}

Bevor in Kapitel 3.1.1 der internationale Forschungsstand zum Zusammenhang verschiedener Mediennutzungsparameter mit schulischen Leistungen näher dargestellt wird, soll zunächst der Begriff „Schulleistung“ näher erläutert und ein Rahmenmodell schulischer Leistungen vorgestellt werden. Nach Helmke und Weinert (1997) sowie Schrader und Helmke (2008) können Schulleistungen im engeren Sinne als fachspezifische Leistungen verstanden werden, die sich über den Erwerb, die Verfügbarkeit und Nutzung von deklarativem Wissen, prozeduralen Fertigkeiten sowie metakognitiven Kompetenzen kennzeichnen lassen. Diese werden entweder direkt durch Lehrkräfte beurteilt, oder über schulbezogene Aufgaben und Leistungstest erhoben (Helmke \& Weinert, 1997). In der Bildungsforschung wird an Stelle des Begriff der Leistung jedoch zunehmend auf den Kompetenzbegriff zurückgegriffen (vgl. PISA, Baumert et al., 2001; Prenzel et al., 2005; Prenzel et al., 2007; Klieme et al., 2010; vgl. IGLU, Bos et al., 2003; Bos et al., 2007): „Dabei versteht man unter Kompetenzen die bei Individuen verfügbaren oder durch sie erlernbaren kognitiven Fähigkeiten und Fertigkeiten, um bestimmte Probleme zu lösen, sowie die damit verbundenen motivationalen, volitionalen und sozialen Bereitschaften und Fähigkeiten um die Problemlösungen in variablen Situationen erfolgreich und verantwortungsvoll nutzen zu kön- 
nen“ (Weinert, 2002, S. 27-28). Im Schulkontext sind dies fachliche Kompetenzen (z. B. Beherrschung von Sprachen), fachübergreifende Kompetenzen (z. B. Problemlösen) sowie allgemeine Handlungskompetenzen, „die neben kognitiven auch soziale, motivationale, volitionale und oft auch moralische Kompetenzen enthalten und es erlauben, erworbene Kenntnisse und Fertigkeiten in sehr unterschiedlichen Lebenssituationen erfolgreich, aber auch verantwortlich zu nutzen“ (Weinert, 2002, S. 28). Somit schließt das Konzept der Kompetenz auch die zum Einsatz der kognitiven Fähigkeiten und Fertigkeiten notwendigen Bedingungsfaktoren ein und grenzt sich in diesem Punkt von der engeren Definition der meist lehrplanbezogenen Leistung ab. ${ }^{86}$

Bedingungen schulischer Leistungen. Seien es Schulleistungen (z. B. erfasst über Noten) oder Kompetenzen (z. B. Lesekompetenzen, mathematische Grundbildung, naturwissenschaftliche Grundbildung, Fähigkeit zum selbstregulierten Lernen, soziale und kommunikative Kompetenzen), beide sind von einer Vielzahl weiterer Faktoren abhängig, weswegen bei der Erklärung interindividueller Schulleistungsunterschiede durchgehend multikausale Erklärungsmodelle zugrunde gelegt werden. Schrader und Helmke (2008) unterscheiden aufbauend auf dem wohl bekanntesten Erklärungsmodell schulischer Leistungen von Helmke und Weinert (Helmke \& Weinert, 1997; vgl. Weinert \& Helmke, 1997) folgende Einflussgrößen: den Kontext und außerschulische Bedingungen (Bildungssystem und kultureller Kontext, sozialer und familiärer Hintergrund, Gleichaltrige, Medien, Schul- und Klassenkontext), Individuelle Bedingungsfaktoren (Intelligenz, Vorwissen, Metakognition und Lernstrategien, Motivation, Volition, Aufmerksamkeit) und den Unterricht.

„Leistungen sind [dabei] das Ergebnis einer Wirkungskette, die von distalen, den Lernerfolg nur indirekt beeinflussenden, zu proximalen, unmittelbar auf den Lernerfolg einwirkenden Faktoren verläuft. Da Leistungen immer das Ergebnis von Aktivitäten des einzelnen Lernenden sind, weisen prozessnahe

86 Zur Diskussion Leistung vs. Kompetenz äußert sich Weinert wie folgt: „Was aber soll eigentlich gemessen werden? Fachliche oder fachübergreifende Leistungen, Wissen oder Können, kognitive Kompetenzen oder Handlungskompetenzen? An der Beantwortung dieser Frage hat sich in jüngster Zeit viel Streit entzündet. Dabei geht es vor allem um die Behauptung, dass fachlichen Leistungen in Zukunft eine immer geringere, fachübergreifenden Kompetenzen aber eine ständig wachsende Bedeutung zukommen wird. Diese These ignoriert die gut belegte Tatsache, dass Fächer nicht beliebige Wissenskonglomerate darstellen, sondern sachlogische Systeme, die Schüler aktiv und konstruktiv erwerben müssen, wollen sie schwierige inhaltliche Phänomene und Probleme tiefgründig verstehen und soll zukünftiges Lernen durch Transferprozesse erleichtert werden. Das gilt auch für die Bearbeitung von Aufgaben, die zwar in nicht-fachlichen Kontexten eingebettet, aber ihrem Wesen nach fachlicher Natur sind." (Weinert, 2002, S. 27). Auf der anderen Seite könnte man aber auch fragen, ob die in PISA und IGLU durchgeführten Kompetenztests, die als Leistungstest in einer Prüfungssituation durchgeführt werden, Leistungen unter einem anderen Namen erheben. 
individuelle Schülermerkmale wie kognitive, motivationale oder volitionale Merkmale den engsten Bezug zur Leistung auf. Die Personenmerkmale werden von Prozessmerkmalen des Unterrichts [...] beeinflusst, denen wiederum Merkmale des Schul- und Klassenkontextes [...] sowie Personenmerkmale der Lehrperson vorgeschaltet sind. Daneben spielen außerschulische Einflussfaktoren wie familiäre Lernumwelt, Medien oder Gleichaltrige eine Rolle.“ (Schrader \& Helmke, 2008)

Ein sehr guter Überblick zur differenziellen Bedeutsamkeit der unterschiedlichen Bedingungsfaktoren findet sich beispielsweise bei Helmke und Weinert (1997), bei Weinert und Helmke (1997) oder bei Schrader und Helmke (2008). Die genauen Zusammenhänge zwischen diesen einzelnen Faktoren in deren gemeinsamen Erklärung von Schulleistung sind jedoch weitgehend unklar (vgl. Helmke \& Weinert, 1997). Im Geflecht der unterschiedlichen Schulleistungsdeterminanten wird den kognitiven, motivationalen und volitionalen Merkmalen auf Seiten des Individuums die größte Erklärungskraft schulischer Leistungen zugeschrieben (die durchschnittliche Korrelation zwischen Schulleistung und Intelligenz liegt bei $r=.5$ ).

Bei der Konzeption des Berliner Längsschnitts Medien wurde ein Rahmenmodell der Bedingungsfaktoren schulischer Leistungen der aktuellen nationalen Schulleistungsforschung zugrunde gelegt (siehe Abbildung 48, in grün dargestellt sind jene Bereiche, die in der Studie abgedeckt werden konnten). Dies stellt eine Verknüpfung der Modelle aus dem Programme for International Student Assessment - PISA (z. B. Baumert et al., 2002) und der Internationalen Grundschul-Lese-Untersuchung - IGLU (z. B. Bos et al., 2003) dar. Beide Modelle gehen in ihren Ursprüngen auf Haertel, Walberg und Weinstein (1983), auf Wang, Haertel und Walberg (1993) sowie auf Helmke und Weinert (1997) zurück. Dem Verständnis von Schrader und Helmke (2008) folgend ist die Medienumwelt als Bedingungsfaktor des sozialen Kontextes in diesem multifaktoriellen Erklärungsmodell eher als distaler Faktor zu betrachten, welcher die Schulleistungen der Individuen nur indirekt beeinflusst (vgl. PISA, Baumert et al., 2001; vgl. Wittwer \& Senkbeil, 2008). Im Vergleich hierzu wären beispielsweise die kognitiven Schülermerkmale ein proximaler Einflussfaktor. Schrader und Helmke (2008) fassen den Begriff Medienumwelt weit und subsumieren darunter neben der privaten auch die schulische Medienumwelt und neben der Nutzung problematischer Inhalte auch die Nutzung förderlicher Inhalte. ${ }^{87}$ Dagegen soll hier aus-

87 So könne nach Schrader und Helmke (2008) das Fernsehen je nach Art der Sendung sowie dem Alter und dem Entwicklungsstand der Kinder eine positive wie negative Wirkung entfalten. Auf der anderen Seite böte der Einsatz von Computern insbesondere die Möglichkeit einer stärkeren Individualisierung oder die Anregung interaktiver oder kooperativer Prozesse. 
schließlich eine häusliche, meist unterhaltungsmotivierte (vgl. Kapitel 2) Mediennutzung als Bedingungsfaktor schulischer Leistungen betrachtet werden. Eine derartige inhaltliche Eingrenzung scheint bei einer Einordnung der Medienumwelt unter die außerschulischen Bedingungsfaktoren des sozialen Kontextes gerechtfertigt (eine schulische Mediennutzung müsste zudem den schulinternen Bedingungsfaktoren zugeordnet werden).

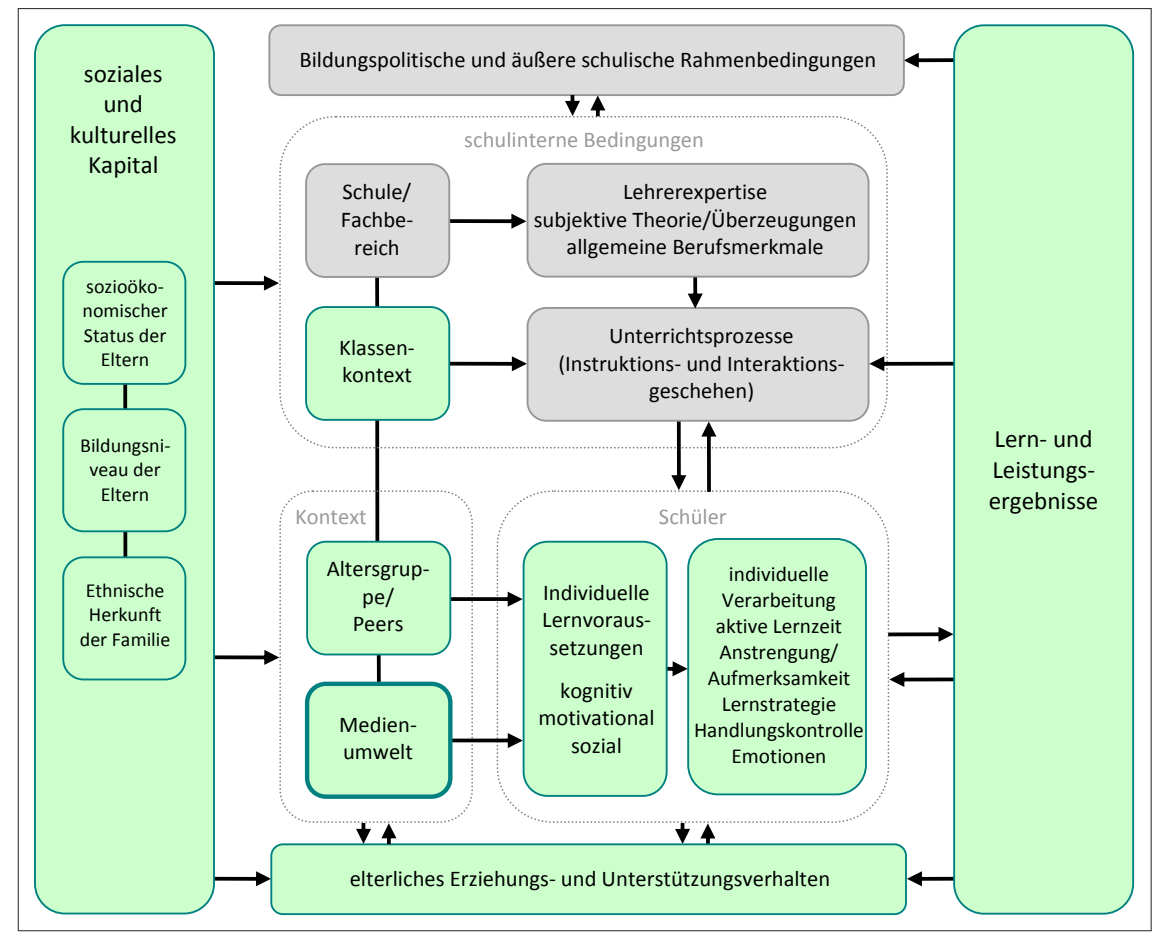

Abbildung 48. Rahmenmodell schulischer Leistungen.

In den internationalen Schulleistungsuntersuchungen, PISA (z. B. Baumert et al., 2002) und $I G L U$ (z. B. Bos et al., 2003) wurde die kindliche oder jugendliche Mediennutzung trotz angenommener Zusammenhänge mit dem elterlichen Erziehungs- und Unterstützungsverhalten, der Peer-Group, den individuellen Lernvoraussetzungen auf Seiten des Schülers oder der Schülerinnen sowie dem sozialen und kulturellen Kapital im Elternhaus (vgl. Baumert et al., 2001), in der Vergangenheit eher stiefmütterlich behandelt. So wurde lediglich die Ausstattung mit Mediengeräten (Baumert et al., 2001) oder die außerschulische PC-Nutzung von Schülerinnen und Schülern (Bos et al., 2003) abgefragt. 
Dass eine derart verkürzte Erfassung der Mediennutzung zu irreführenden Ergebnissen führen kann, zeigten insbesondere Auswertungen der PISA-Daten zum Zusammenhang der individuellen Mediennutzung und Kompetenzen der Schülerinnen und Schüler. So ermittelte die PISA-Studie 2000 beispielsweise zunächst einen positiven Zusammenhang zwischen der heimischen Verfügbarkeit eines Computers und höheren Kompetenzen der Jugendlichen in Mathematik und im Lesen (vgl. OECD, 2001). Die Autoren wiesen jedoch bereits darauf hin, dass die gefundenen korrelativen Zusammenhänge mit Vorsicht zu interpretieren seien, da mögliche Einflussvariablen nicht berücksichtigt wurden und keine Aussage über die Wirkungsrichtung getroffen werden kann. Diese Einschätzung bestätigte eine Re-Analyse der PISA-Daten durch Fuchs und Wößmann (2004), in der sich zeigte, dass sich der von PISA noch vorsichtig berichtete positive $\mathrm{Zu}$ sammenhang zwischen Computerverfügbarkeit zu Hause und Mathematikleistung sowie Lesefähigkeit schon bei Kontrolle des familiären Hintergrundes (elterliche Erziehung, Migrationsstatus, sozioökonomischer Status) innerhalb einer multivariaten Regression umkehrt, d. h. eine Computerverfügbarkeit zu Hause ging mit schlechteren Leistungen einher. Computerbesitz war demnach zu Beginn des Jahrhunderts nach Einschätzung der Autoren ein sehr guter Indikator eines hohen soziökonomischen Status (Fuchs, T. \& Wößmann, 2004). Zudem stellen die Autoren fest, dass der heimische Computer hauptsächlich als Plattform zum Spielen von Computerspielen genutzt wurde und so bei einigen Schülerinnen und Schülern im Sinne einer Zeitverdrängung in direkte Konkurrenz zum schulischen Lernen trat. In ähnlicher Weise wurde auch in der PISA-Studie 2003 zunächst ein positiver Zusammenhang zwischen der heimischen Verfügbarkeit sowie häufigen Nutzung eines Computers und besseren Mathematikleistungen berichtet (OECD, 2006). Dieser Zusammenhang kehrte sich jedoch wiederum bei Kontrolle wichtiger Faktoren wie dem Geschlecht, dem Migrationshintergrund, kognitiven Grundfähigkeiten und der Nutzung anderer Medien (Lesen und Fernsehen) in einer Re-Analyse von Wittwer und Senkbeil (2008) tendenziell (wenn auch statistisch nicht bedeutsam) um. Auch der Konsum von Filmgenres wie z. B. Horror- oder Pornofilmen hatte einen negativen Effekt auf die Problemlösungs- und mathematischen Kompetenzen der Schülerinnen und Schüler (Wittwer \& Senkbeil, 2008).

Zusammengenommen zeigen diese beiden Beispiele recht anschaulich, dass bei der Betrachtung des Zusammenhangs von Mediennutzung und Schulleistung sowohl eine differenzierte Erfassung verschiedener Mediennutzungsparameter neben einer reinen Medienausstattung sind hier Nutzungszeiten und vor allem Nutzungsinhalte verschiedener Medien zu nennen - als auch die Berücksichtigung der wichtigsten anderen Einflussfaktoren von essenzieller Bedeutung sind. 
Dies ist bei der Darstellung der Forschungsergebnisse zum Zusammenhang von Mediennutzung und Schulleistung stets zu berücksichtigen.

\subsubsection{Forschungsergebnisse zum Zusammenhang Mediennutzung und Schulleistung}

Seit der zunehmenden Verfügbarkeit des Fernsehens beginnend in den 1950er Jahren sowie von Computerspielen in den 1980er Jahren, wurden die Zusammenhänge zwischen der Nutzung elektronischer Medien (in der chronologischen Reihenfolge Fernsehen, Video, Computerspiele, später Internet) und schulischen Leistungsindikatoren in der internationalen Medienwirkungsforschung mit unterschiedlichen Untersuchungsdesigns fortwährend beleuchtet. Dabei konnte die frühe Medienwirkungsforschung der 1950er bis 1970er Jahre meist keine oder statistisch nur wenig bedeutsame Zusammenhänge zwischen der häuslichen Fernsehnutzung und schulischen Leistungen beobachten - auch bei Kontrolle wichtiger Einflussfaktoren wie z. B. Intelligenz und sozioökonomischem Status (vgl. Gaddy, 1986; für eine Längsschnittstudie mit Daten der 1960er Jahre siehe Gortmaker, Salter, Walker \& Dietz, 1990). So berichten auch Williams, Haertel, Haertel und Walberg (1982) in ihrer Metaanalyse, in der 23 internationale Studien der Veröffentlichungsjahrgänge 1954 bis 1980 mit insgesamt 274 Korrelationen zwischen Fernsehnutzungszeiten und schulischen Leistungen berücksichtigt wurden, lediglich eine schwache mittlere Korrelation von $r=.05$.

Seit den 1980er Jahren mehren sich jedoch Studien, die einen eindeutig negativen Zusammenhang zwischen Variablen der Mediennutzung und schulischen Leistungen nachweisen können (vgl. Ennemoser \& Schneider, 2007). Den Beginn machte dabei eine der wohl bekanntesten Studien der Fernsehwirkungsforschung von Williams und Kollegen (Williams, T. M., 1986), die in ihrer Studie Familien einer kanadischen Gemeinde, in der erst 1973 Fernsehempfang verfügbar wurde, vor und nach der Einführung des Fernsehens im Vergleich zu zwei weiteren Kommunen mit Fernsehempfang untersuchen konnten. Als Ergebnis zeigten sich negative Effekte des Fernsehens auf den Erwerb von Lesefähigkeiten, Leseverhalten und kreativem Denken. Neuman erweiterte in den späten 1980er Jahren die korrelativen Befunde der Metaanalyse von Williams, Haertel, Haertel und Walberg (1982) und konnte an US-Studien der Jahrgänge 1978 bis 1983 unter Kontrolle des sozioökonomischen Status im Elternhaus regressionsanalytisch ebenfalls einen Zusammenhang zwischen hohen Fernsehnutzugszeiten und geringeren Lesefähigkeiten im Sinne eines kurvilinearen Zusammenhangs nachweisen (Neuman, 1988). In ihrem kritischen Review der Fernsehwirkungsforschung kommen Comstock und Scharrer (1999) ferner für die 1980er 
Jahre anhand von Daten des California Assessment Programs (CAP), in welchem 1980, 1982 und 1986 Leistungen in Mathematik, Lesen und Schreiben sowie die Fernsehnutzungszeiten von 282.000 Sechstklässlern und 227.000 Zwölftklässlern erhoben wurden, zu dem Schluss, dass für alle drei erhobenen Leistungsbereiche auch bei Kontrolle des sozioökonomischen Status eine negative Beziehung zur heimischen Fernsehnutzungszeit konstatiert werden muss.

Mitte der 90er Jahre zeigten Koolstra, van der Voort und van der Kamp (1997) in ihrer Längsschnittstudie mit einem Panel von 1.050 Grundschülern und Grundschülerinnen, abweichend von den Befunden von Gaddy (1986) und Gortmaker et al. (1990) die auch längsschnittlich keinen Zusammenhang zwischen häuslichen Fernsehnutzungszeiten und schulischen Leistungen finden konnten, dass insbesondere eine hohe Nutzung von Unterhaltungssendungen - bei Kontrolle der Intelligenz sowie des sozioökonomischen Status der Kinder - längsschnittlich mit einem schlechterem Leseverständnis zusammenhing. Durch die Gegenüberstellung von Unterhaltungs- und Edutainmentprogrammen rückt in dieser Längsschnittstudie auch die Bedeutung der genutzten Medieninhalte bei der Betrachtung des Zusammenhangs schulischer Leistungen und Medienkonsums in den Vordergrund. Razel (2001) fasste schließlich in seiner Metaanalyse mit über 1.000.000 Schülerinnen und Schülern 305 Korrelationskoeffizienten zu Zusammenhängen zwischen Fernsehnutzungszeiten und Schulleistung (Lesen, Mathematik und Naturwissenschaften) aus sechs internationalen Studien der Jahrgänge von 1986 bis 1998 zusammen (vgl. Tabelle 45) und konnte zeigen, dass 90 Prozent der berichteten Korrelationen zwischen Fernsehzeit und Schulleistung negativ ausfielen.

Die Forschung zur Wirkung von Computerspielen auf schulische Leistungen entwickelte sich verstärkt - analog zur technischen Entwicklung - ab den späten 1990er Jahren. Es gab aber bereits Mitte der 1980er Jahre, in einer Zeit also, als Computerspiele noch in ihren Kinderschuhen steckten, weniger verbreitet und mit heutigen Produkten nicht vergleichbar waren, vereinzelte Forschungsbemühungen. So konnten Harris und Williams (1985) in einer Studie mit 152 Schülerinnen und Schülern der neunten bis zwölften Klasse einen negativen Zusammenhang zwischen den Computerspielzeiten $(r=-.28)$ sowie dem für Computerspiele ausgegebenen Geld $(r=-.20)$ und der Englischnote berichten. Lin und Lepper fanden 1987 in einer Studie mit 210 Schülerinnen und Schülern der vierten bis sechsten Klasse negative Korrelationen zwischen der Nutzung von Arkadespie$\operatorname{len}^{88}(r=-.24)$ - vor allem bei Jungen $(r=-.20)$ - sowie der heimischen Computerspielnutzung $(r=-.16)$ - vor allem bei Mädchen $(r=-.19)-$ und der durch Lehrkräfte eingeschätzten schulischen Kompetenz (Lin, S. \& Lepper, 1987). En-

88 Arkadespiele können (in öffentlichen Spielhallen) an Spielautomaten gegen Geldeinwurf gespielt werden. 
de der 1990er berichten Van Schie und Wiegmann (1997) schließlich in einer weiteren Querschnittsstudie mit 346 niederländischen Schülerinnen und Schülern der siebten und achten Klasse unter Kontrolle der Intelligenz über keinen statistisch bedeutsamen Zusammenhang zwischen der Nutzung von Computerspielen, erhoben in einem Freizeittagebuch, und schulischen Leistungen.

Aufgrund des starken Wandels und der ständigen Weiterentwicklung im Bereich der elektronischen Unterhaltungsmedien, insbesondere auf dem Gebiet der Computerspiele, soll im Folgenden, um die gegenwärtige Relevanz interaktiver Unterhaltungsmedien für schulische Leistungsfähigkeit aufzeigen zu können, auf aktuelle Studien der internationalen Medienwirkungsforschung der letzten zehn Jahre zurückgegriffen werden. Grundlage hierfür war eine Literaturrecherche einschlägiger Studien der Jahre 2001 bis 2011, wobei nur Studien in die Übersicht mit aufgenommen wurden, die zumindest eine kindliche oder jugendliche Substichprobe beinhalteten. ${ }^{89}$

Bei den 29 aufgeführten Studien handelt es sich um eine Metaanalyse (Razel, 2001), 2 Überblicksartikel (Subrahmanyam, Greenfield, Kraut \& Gross, 2001; Kirkorian, Wartella \& Anderson, 2008), 18 Querschnittsstudien (Roe, Eggermont \& Minnebo, 2001; Gentile, Lynch, Linder \& Walsh, 2004; Shin, 2004; Hunley et al., 2005; Borzekowski \& Robinson, 2005; Roberts, Foehr \& Rideout, 2005; Chan \& Rabinowitz, 2006; Sharif \& Sargent, 2006; Anand, 2007; Mößle et al., 2007; Ip, Jacobs \& Watkins, 2008; Eow, Ali, Mahmud \& Baki, 2009; Hastings, E. C. et al., 2009; Jaruratanasirikul, Wongwaitaweewong \& Sangsupawanich, 2009; Skoric, Teo \& Neo, 2009; Ferguson, 2010; Schittenhelm, Ennemoser \& Schneider, 2010; Ennemoser \& Schneider, 2009; Baier \& Pfeiffer, 2011) sowie 8 Längsschnittstudien (Anderson, D. R., Huston, Schmitt, Linebarger \& Wright, 2001; Schiffer, Ennemoser \& Schneider, 2002; Ennemoser, 2003; Ennemoser, Schiffer, Reinsch \& Schneider, 2003; Ennemoser \& Schneider, 2007, 2009; Hancox, Milne \& Poulton, 2005; Zimmerman \& Christakis, 2005; Johnson, Cohen, Kasen \& Brook, 2007; Hofferth, 2010; Sharif, Wills \& Sargent, 2010; Baier \& Pfeiffer, 2011). Die genauen Details der Studien (Stichprobe, abhängige und unabhängige Variablen, Auswertungsmethodik - soweit berichtet und Befunde) können Tabelle 45 (Metaanalysen und Reviews), Tabelle 46 (Querschnittsstudien) und (Längsschnittstudien) entnommen werden.

89 Für den Zeitraum 2001 bis 2011 wurden die Datenbanken PsychInfo, Pubmed und google scholar mit den Suchbegriffen Mediennutzung, Fernseh, TV, Videospiel, Computerspiel, media use, television, tv, video games, computer games, jeweils in Kombination (UND / AND) mit Schulerfolg, Schulleistung, Schulnoten, academic achievement, school achievement durchsucht. Als Treffer galten Querschnitts- und Längsschnittuntersuchungen, an denen Kinder und Jugendliche teilgenommen haben, sowie Reviews oder Metaanalysen, die sich ebenfalls mit dieser Population befassten. 
Vor einem kurzen Ergebnisüberblick seien zwei methodische Kritikpunkte vorweggenommen. Zum einen wurde häufig auf die Nutzungszeiten der verschiedenen Medien fokussiert, ohne die genutzten Inhalte zu berücksichtigen. Die Wichtigkeit der genutzten Inhalte bei der Untersuchung des Zusammenhangs von Mediennutzung und Schulleistung konnte jedoch bereits von Koolstra, van der Voort und van der Kamp (1997) in ihrer Längsschnittstudie aufgezeigt werden. Zudem ist eine ausschließliche Fokussierung auf entweder Fernsehnutzungs- oder Computerspielnutzungsvariablen problematisch, da diese das Alltagserleben der Kinder und Jugendlichen nur verkürzt darstellen würde und zudem unterschiedliche Medien eine differenzielle Wirkung entfalten können. In den 29 aufgeführten Studien der letzten 10 Jahre zum Zusammenhang von Mediennutzung und Schulleistung wurden nur in elf Studien die Auswirkungen erhöhter Medienzeiten und (v.a. gewalthaltiger) Medieninhalte gleichzeitig berücksichtigt (1 Überblicksartikel, Kirkorian et al., 2008; 6 Querschnittsstudien, Gentile et al., 2004; Mößle et al., 2007; Ip et al., 2008; Hastings, E. C. et al., 2009; Ferguson, 2010; Baier \& Pfeiffer, 2011; 4 Längsschnittstudien, Anderson, D. R. et al., 2001; Schiffer et al., 2002; Ennemoser, 2003; Ennemoser et al., 2003; Ennemoser \& Schneider, 2007, 2009; Sharif et al., 2010). Wurden Inhalte und Nutzungszeiten gleichzeitig im Längsschnitt erhoben, geschah dies nur für das Medium Fernsehen, nicht jedoch für Computerspiele. Unter den genannten Studien betrachteten ferner nur 12 Fernseh- sowie Computerspielkonsum gemeinsam in ihrer Bedeutung für eine Schulleistungsentwicklung ( 9 Querschnittsstudien, Roe et al., 2001; Gentile et al., 2004; Borzekowski \& Robinson, 2005; Roberts et al., 2005; Chan \& Rabinowitz, 2006; Sharif \& Sargent, 2006; Anand, 2007; Mößle et al., 2007; Baier \& Pfeiffer, 2011; 3 Längsschnittstudien, Hofferth, 2010; Sharif et al., 2010; Baier \& Pfeiffer, 2011). Insgesamt gibt es nur vier Studien, welche sowohl die genutzten Inhalte als auch die genutzten Zeiten für sowohl Computerspiele als auch das Fernsehen erfassten. Dies sind drei Querschnittsstudien (Gentile et al., 2004; Mößle et al., 2007; Baier \& Pfeiffer, 2011) und eine Längsschnittstudie (Baier \& Pfeiffer, 2011).

Zusammengefasst liefern die genannten nationalen wie internationalen Studien mittels variierender Untersuchungs- (Querschnitt, Längsschnitt, Metaanalyse) und Auswertungsmethodik (Korrelation, t-Test, Varianzanalyse, Regressionsanalyse, Strukturgleichungsmodelle bzw. Pfadanalysen sowie Mehrebenenanalysen) zahlreiche Belege dafür, dass sich die Schulleistungen von Kindern und Jugendlichen mit steigendem Fernsehkonsum (Razel, 2001; Subrahmanyam et al., 2001; Kirkorian et al., 2008; Roe et al., 2001; Gentile et al., 2004; Shin, 2004; Borzekowski \& Robinson, 2005; Roberts et al., 2005; Chan \& Rabinowitz, 2006; Sharif \& Sargent, 2006; Anand, 2007; Mößle et al., 2007; Schittenhelm et al., 2010; Ennemoser \& Schneider, 2009; Baier \& Pfeiffer, 2011; Anderson, D. R. et al., 
2001; Schiffer et al., 2002; Ennemoser, 2003; Ennemoser et al., 2003; Ennemoser \& Schneider, 2007; Hancox et al., 2005; Zimmerman \& Christakis, 2005; Johnson et al., 2007; Sharif et al., 2010) oder steigendem Computerspielkonsum (Roe et al., 2001; Gentile et al., 2004; Borzekowski \& Robinson, 2005; Roberts et al., 2005; Chan \& Rabinowitz, 2006; Sharif \& Sargent, 2006; Anand, 2007; Mößle et al., 2007; Ip et al., 2008; Eow et al., 2009; Hastings, E. C. et al., 2009; Jaruratanasirikul et al., 2009; Skoric et al., 2009; Baier \& Pfeiffer, 2011; Hofferth, 2010; Sharif et al., 2010) verschlechtern. Korrelativ bewegen sich die statistisch bedeutsamen Zusammenhänge zwischen $r=-.10$ und $r-.31$ und somit im kleinen bis mittleren Bereich. Lediglich jeweils zwei Studien berichten über keinen statistisch bedeutsamen Zusammenhang zwischen Fernsehnutzungszeiten (Ferguson, 2010; Hofferth, 2010) bzw. Computerspielnutzungszeiten (Hunley et al., 2005; Ferguson, 2010) und Indikatoren der Schulleistung. Zum Teil weisen diese statistisch nicht bedeutsamen Zusammenhänge jedoch auch in die Richtung einer negativen Wirkung (Hunley et al., 2005; Ferguson, 2010).

Zugleich gibt es auch Belege dafür, dass den konsumierten Medieninhalten am Fernseher (Kirkorian et al., 2008; Mößle et al., 2007; Baier \& Pfeiffer, 2011; Anderson, D. R. et al., 2001; Schiffer et al., 2002; Ennemoser, 2003; Ennemoser et al., 2003; Ennemoser \& Schneider, 2007, 2009; Sharif et al., 2010) sowie am PC oder der Spielkonsole (Gentile et al., 2004; Mößle et al., 2007; Ip et al., 2008; Hastings, E. C. et al., 2009; Baier \& Pfeiffer, 2011) eine eigenständige Bedeutung zur Erklärung schlechterer Schulleistungen zukommt. Korrelativ sind die statistisch bedeutsamen Zusammenhänge wiederum im kleinen bis mittleren Bereich anzuordnen, $r=-.10$ und $r-.34$. Lediglich Ferguson (2010) berichtet über keine statistisch bedeutsamen Zusammenhänge zwischen Fernseh- bzw. Computerspielinhalten und Indikatoren der Schulleistung.

In verschiedenen Studien zeigte sich zudem, dass einerseits der beobachtete negative Zusammenhang zwischen Variablen der Mediennutzung und schulischen Leistungen auch bei Berücksichtigung weiterer (Moderator-)Variablen (wie z. B. dem IQ der Kinder oder dem sozialen und kulturellen Kapital) bestehen bleibt. Andererseits verdeutlicht dies aber auch, dass die Nutzung elektronischer Bildschirmmedien nur ein weiterer Einflussfaktor im Erklärungsmodell schulischer Leistungen ist (vgl. auch Abbildung 48). In insgesamt 12 (von 18) Querschnittsstudien und allen 8 Längsschnittstudien wurde mindestens eine weitere Einflussvariable betrachtet, am häufigsten das Geschlecht der Kinder sowie das soziale und kulturelle Kapital:

- das Geschlecht der Kinder (Roe et al., 2001; Gentile et al., 2004; Borzekowski \& Robinson, 2005; Mößle et al., 2007; Hastings, E. C. et al., 2009; Jaruratanasirikul et al., 2009; Baier \& Pfeiffer, 2011; Anderson, D. R. et al., 2001; 
Ennemoser, 2003; Ennemoser \& Schneider, 2007, 2009; Schiffer et al., 2002; Hancox et al., 2005; Johnson et al., 2007)

- das soziale und kulturelle Kapital (Roe et al., 2001; Borzekowski \& Robinson, 2005; Sharif \& Sargent, 2006; Mößle et al., 2007; Jaruratanasirikul et al., 2009; Skoric et al., 2009; Baier \& Pfeiffer, 2011; Anderson, D. R. et al., 2001; Ennemoser, 2003; Ennemoser \& Schneider, 2007, 2009; Schiffer et al., 2002; Zimmerman \& Christakis, 2005; Johnson et al., 2007)

- Intelligenz (Ennemoser \& Schneider, 2009; Schittenhelm et al., 2010; Ennemoser, 2003; Ennemoser \& Schneider, 2007; Schiffer et al., 2002; Zimmerman \& Christakis, 2005)

- Aufmerksamkeit (Ennemoser \& Schneider, 2009; Schittenhelm et al., 2010; Johnson et al., 2007)

- die Sprachfähigkeit bei Einschulung (Mößle et al., 2007)

- die Medienausstattung (Borzekowski \& Robinson, 2005; Mößle et al., 2007)

- andere Freizeitaktivitäten (Roe et al., 2001; Baier \& Pfeiffer, 2011)

- Zeit für Hausaufgaben (Shin, 2004; Borzekowski \& Robinson, 2005)

- Zeit für Lesen (Roe et al., 2001; Shin, 2004; Borzekowski \& Robinson, 2005)

- die elterliche Mediennutzung (Gentile et al., 2004)

- die elterliche Medienerziehung (Mößle et al., 2007; Hastings, E. C. et al., 2009)

- die elterliche Erziehung (Sharif \& Sargent, 2006; Baier \& Pfeiffer, 2011; Johnson et al., 2007; Sharif et al., 2010)

- impulsives Verhalten (Shin, 2004)

- Verhaltensprobleme (Hancox et al., 2005; Sharif et al., 2010)

- Trait-Feindseligkeit (Gentile et al., 2004)

- Selbstwert (Sharif \& Sargent, 2006)

- Selbstkontrolle (Sharif et al., 2010)

- Familienstand der Eltern bzw. Scheidungskinder (Jaruratanasirikul et al., 2009)

- Phonologisches Bewusstheit (Ennemoser, 2003; Ennemoser \& Schneider, 2007, 2009; Schiffer et al., 2002)

- Sensation Seeking (Sharif et al., 2010)

Die differenzielle Bedeutung der unterschiedlichen Variablen bei der Erklärung schulischer Leistungen konnte dabei z. B. von Mößle und Kollegen (Mößle et al., 2007) veranschaulicht werden (siehe Abbildung 49). Den größten Einfluss auf die Schulleistungen der Kinder hat, wie zu erwarten, der Bildungshintergrund der Eltern, dem auch eine wesentliche Bedeutung für die Ausstattung der Kinderzimmer mit Mediengeräten zukommt: Je höher die formale Bildung im Elternhaus, desto unwahrscheinlicher ist es, dass die Kinder im eigenen Zimmer über Fernseher und Spielkonsole verfügen. Als stärkster Belastungsfaktor für die 
Schulleistungen erweist sich der Konsum problematischer Medieninhalte. Erhöhte Medienzeiten bedingen auch für sich genommen schlechtere Schulleistungen. Alle „Medienfaktoren“ sind stärker bei Jungen ausgeprägt. Als Moderatoren zeigen sich ein aktive Medienerziehung der Eltern sowie ein positives, gewaltfreies Familienklima.

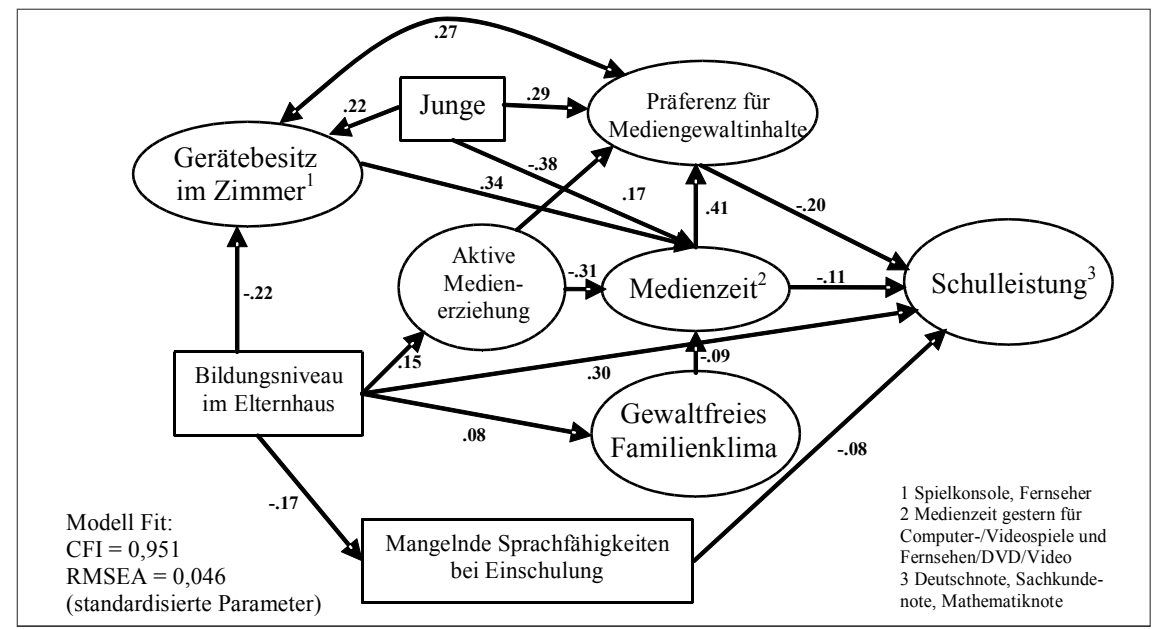

Abbildung 49. Strukturgleichungsmodell zum Einfluss von Mediennutzung auf Schulleistung aus Mößle et al. (2007).

Zudem zeigten sich bei der Berücksichtigung verschiedener Einflussvariablen in unterschiedlichen Studien zum Teil subgruppenspezifische Effekte, die zusammengefasst aber kein einheitliches Bild zur Beurteilung, welche Schülerinnen und welche Schüler insbesondere als gefährdet erscheinen, ergeben. So berichten Ennemoser und Kollegen (Ennemoser, 2003; Ennemoser \& Schneider, 2007, 2009; Schiffer et al., 2002) besondere Beeinträchtigungen in der Gruppe der weniger intelligenten Kinder, Hancox und Kollegen (Hancox et al., 2005) dagegen in der Gruppe der Kinder mit mittlerem IQ. Mößle und Kollegen (Mößle et al., 2007) berichten schließlich zwar über eine besondere Gefährdung durch problematisches Mediennutzungsverhalten für Kinder aus soziokulturell schwächer gestellten Familien, ergänzen aber gleichzeitig, dass die gefundenen Medieneffekte insbesondere auch bei Schülerinnen und Schülern aus Familien mit hohem soziokulturellen Status, welche ein zeitlich und inhaltlich problematisches Mediennutzungsverhalten zeigen, zu beobachten sind (vgl. hierzu auch Comstock \& Scharrer, 1999). Demzufolge treten die Wirkungen problematischer Mediennutzung auf schulische Leistungen keineswegs lediglich in einer kleinen 
Gruppe von Risikokindern auf. An dieser Stelle muss erwähnt werden, dass die Schlussfolgerung, Mediennutzung an und für sich sei für Kinder schädlich, das Bild verfehlt. Schon die frühe Fernsehwirkungsforschung konnte z. T. auch Erfolge bestimmter pädagogischer Edutainmentprogramme, hier insbesondere der Sesamstraße, für eine Leistungssteigerung (Sprachlernen, bessere Noten) bei Kindern beobachten (vgl. hierzu Anderson, D. R. et al., 2001; Comstock \& Scharrer, 1999).$^{90}$ In Kapitel 2 ist aber bereits deutlich geworden, dass eine häufige Nutzung von Edutainmentprogrammen nicht die häusliche Alltagsmediennutzung der Kinder und Jugendlichen abbildet. Für eine Mediennutzung, welche zeitlich ausufert und durch den Konsum nicht altersadäquater Filme oder Computerspiele gekennzeichnet ist, konnten eindeutig leistungsmindernde Effekte beobachtet werden. Dies veranschaulicht aber noch einmal recht deutlich, dass eine Berücksichtigung inhaltlicher Medienvariablen bei der Betrachtung des Zusammenhangs von Mediennutzung und Schulleistung unerlässlich ist. Dies ist aber noch aus einem zweiten Grund von Bedeutung. Frühe Studien, insbesondere zur Wirkung von Fernsehnutzungszeiten, berichteten zum Teil einen kurvilinearen Zusammenhang zwischen Nutzungszeiten und Schulleistungen: Kinder mit mittleren Mediennutzugszeiten erbrachten demnach bessere Leistungen als Kinder mit hohen oder geringen Mediennutzungszeiten (vgl. Williams, P. A. et al., 1982; Neuman, 1988; siehe auch Anand, 2007 für Computerspiele). Auf der anderen Seite gibt es Autoren, die an diesem Zusammenhang zweifeln. Comstock und Scharrer (1999) verweisen etwa auf den hohen Zusammenhang hoher Fernsehnutzungszeiten und der häufigen Nutzung weniger schulrelevanten Inhalts. Niedrige Fernsehzeiten wären mit einem ,more instrumental and informationoriented use" (Comstock \& Scharrer, 1999, S. 254) verbunden. So berichten Ennemoser und Schneider (2007) in ihrer Längsschnittstudie bei der Betrachtung der Unterhaltungsmediennutzung über keinen kurvilinearen Zusammenhang, sondern über negative lineare Effekte. Somit könnten kurvilineare Zusammenhänge möglicherweise durch die gleichzeitige Berücksichtigung von Mediennutzungszeiten und genutzten Inhalten in lineare Zusammenhänge überführt oder zumindest durch unterschiedliche Inhaltsvorlieben erklärt werden. Auf diesem Wege könnten unter Umständen auch die von Razel (2001) berichteten mit dem Lebensalter geringeren idealen Fernsehzeiten über wechselnde inhaltliche Vor-

90 Für Computerspiele werden als lernförderliche Wirkungen z. B. verbesserte visuelle Aufmerksamkeit (Green \& Bavelier, 2003; Risenhuber, 2004; Castel, Pratt \& Drummond, 2005), verbessertes räumliches Vorstellungsvermögen (Green \& Bavelier, 2006; De Lisi \& Wolford, 2002), verbesserte visuelle Wahrnehmungsleistung (Subrahmanyam \& Greenfield, 1994) und eine Verbesserung computerspielspezifischer Heurismen und Gewinnstrategien (Kraam-Aulenbach, 2000) berichtet (vgl. die Metaanalyse von Meerkerk, Van Den Eijnden, Vermulst \& Garretsen, 2009). Im Gesundheitsbereich wurden zudem Computerspiele speziell zur Steigerung des Gesundheitsverhaltens entwickelt und erfolgreich eingesetzt (vgl. Walsh, 2000) 
lieben hin zu einem höheren Anteil an Unterhaltungsmediennutzung (vgl. Johnson et al., 2007) aufgeklärt werden. Details zu den unterschiedlichen Studien finden sich auf den folgenden Seiten - Tabelle 45 (Metaanalysen und Reviews), Tabelle 46 (Querschnittsstudien), (Längsschnittstudien). 


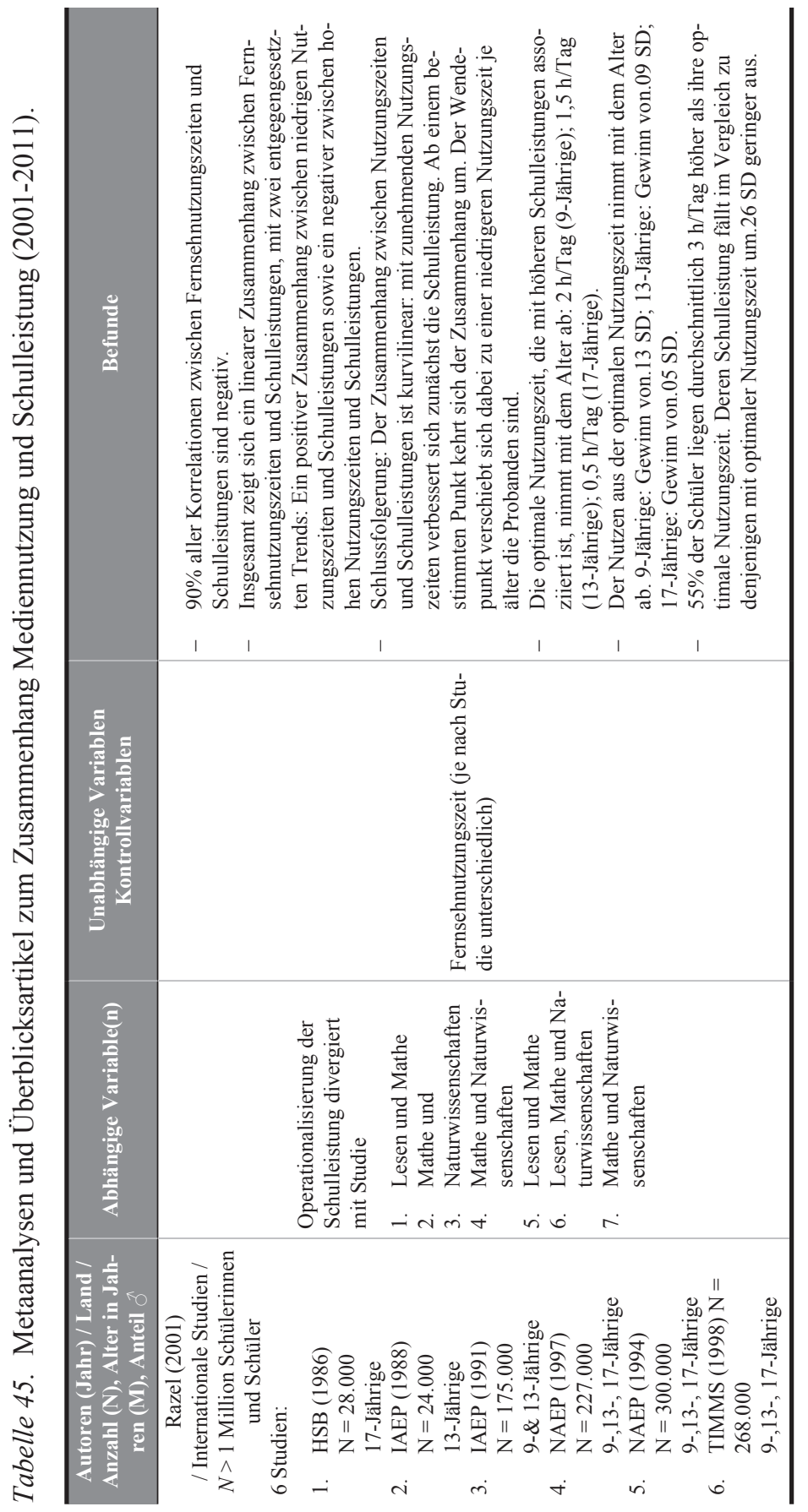




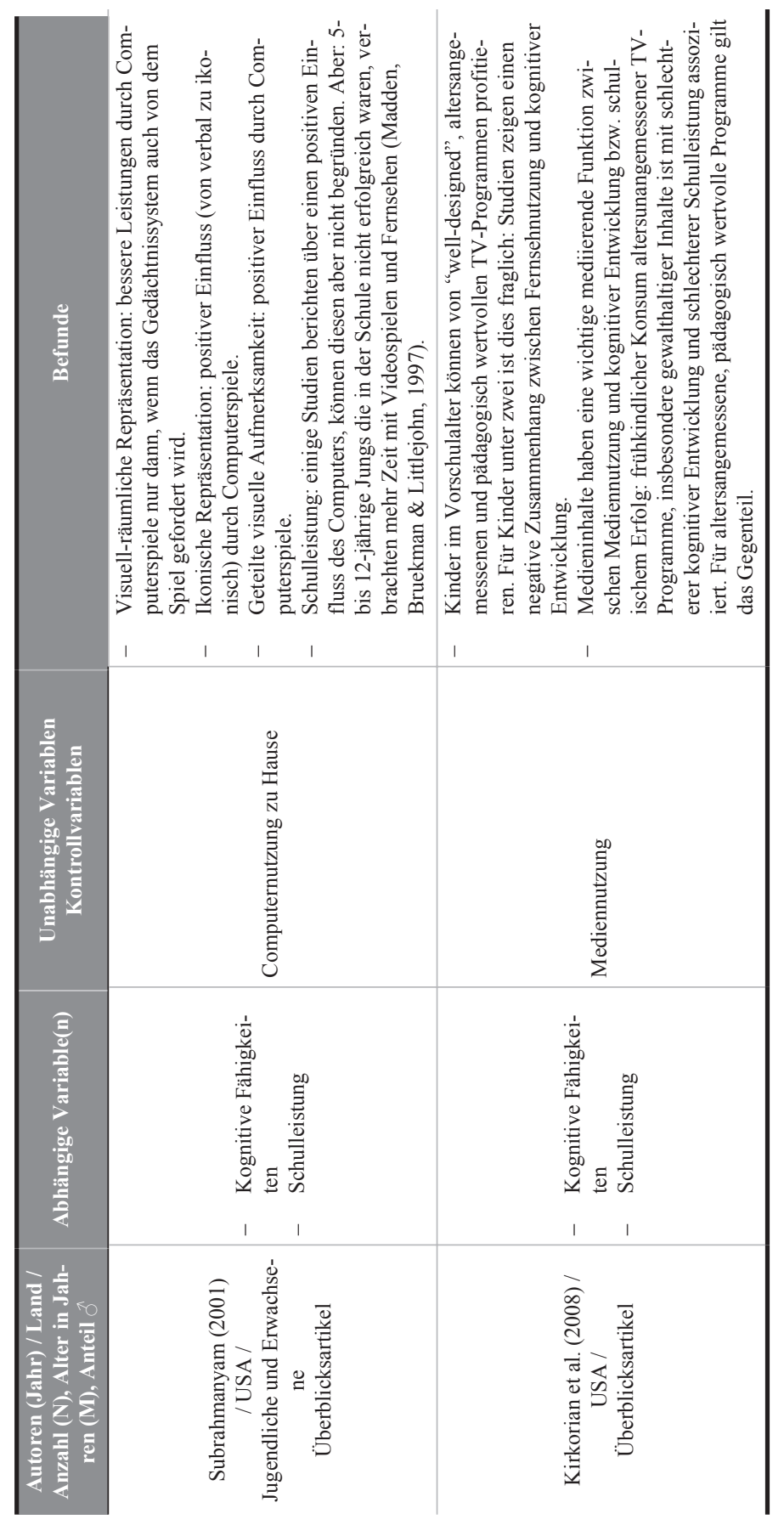




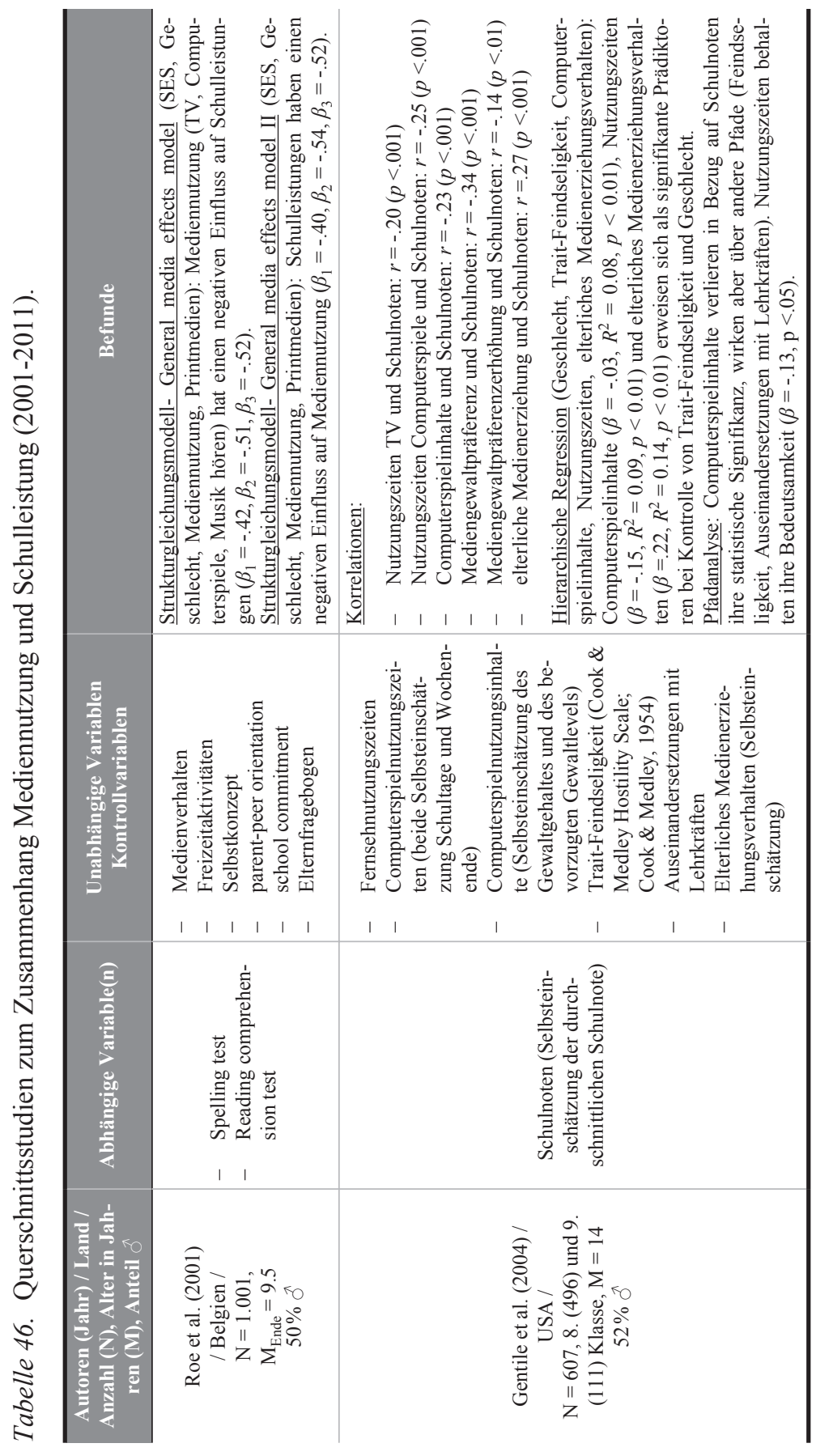




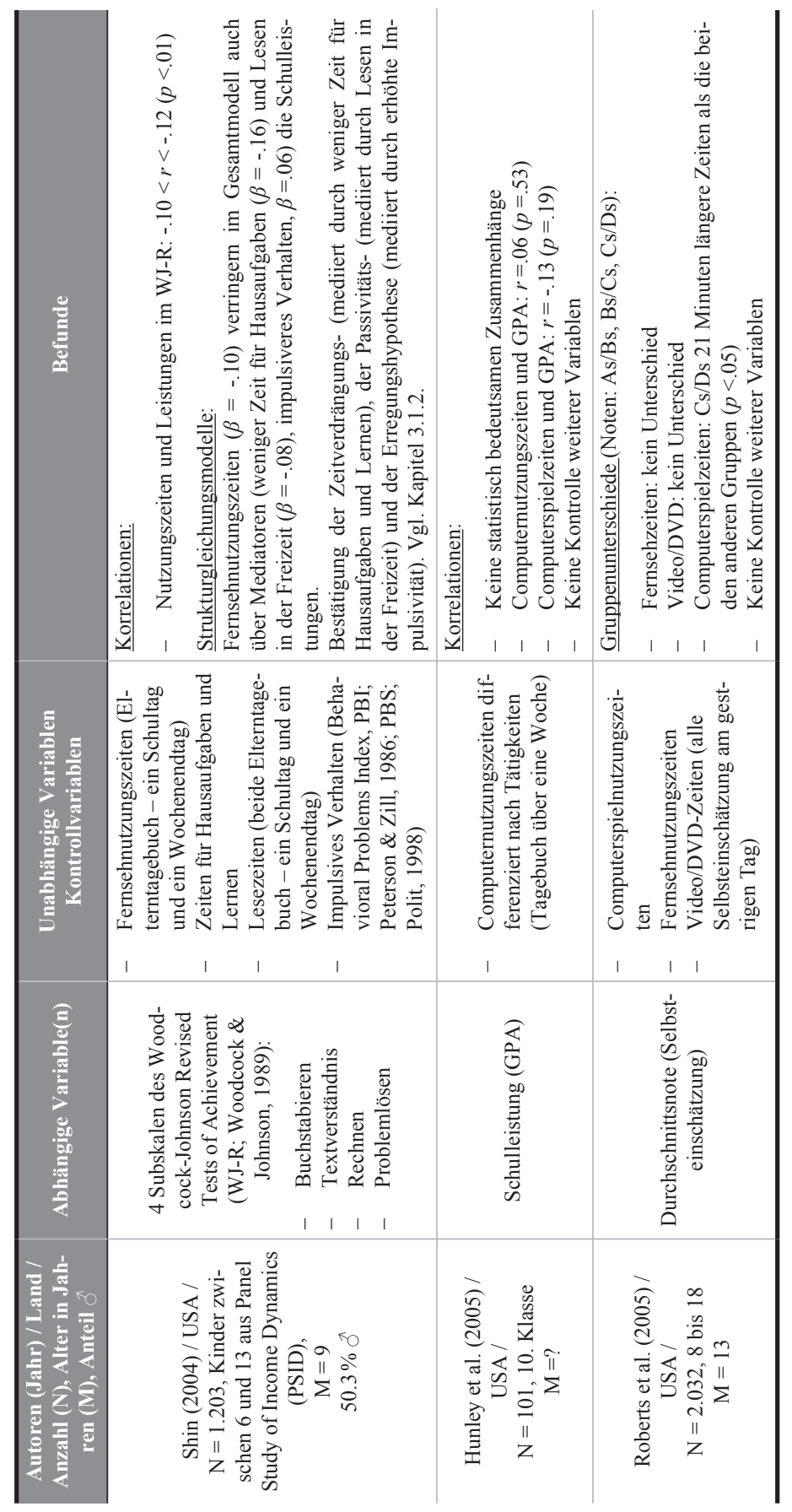




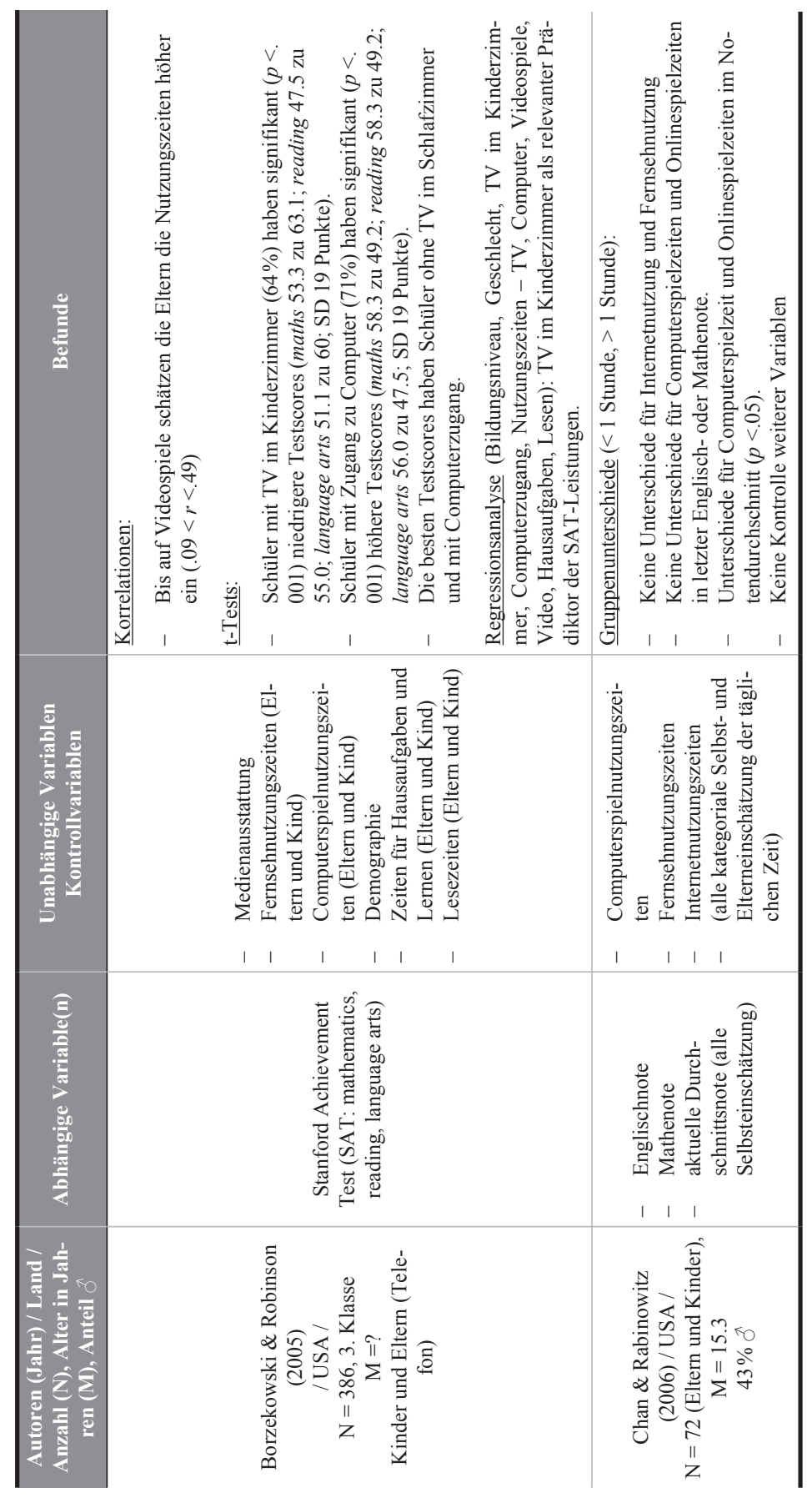




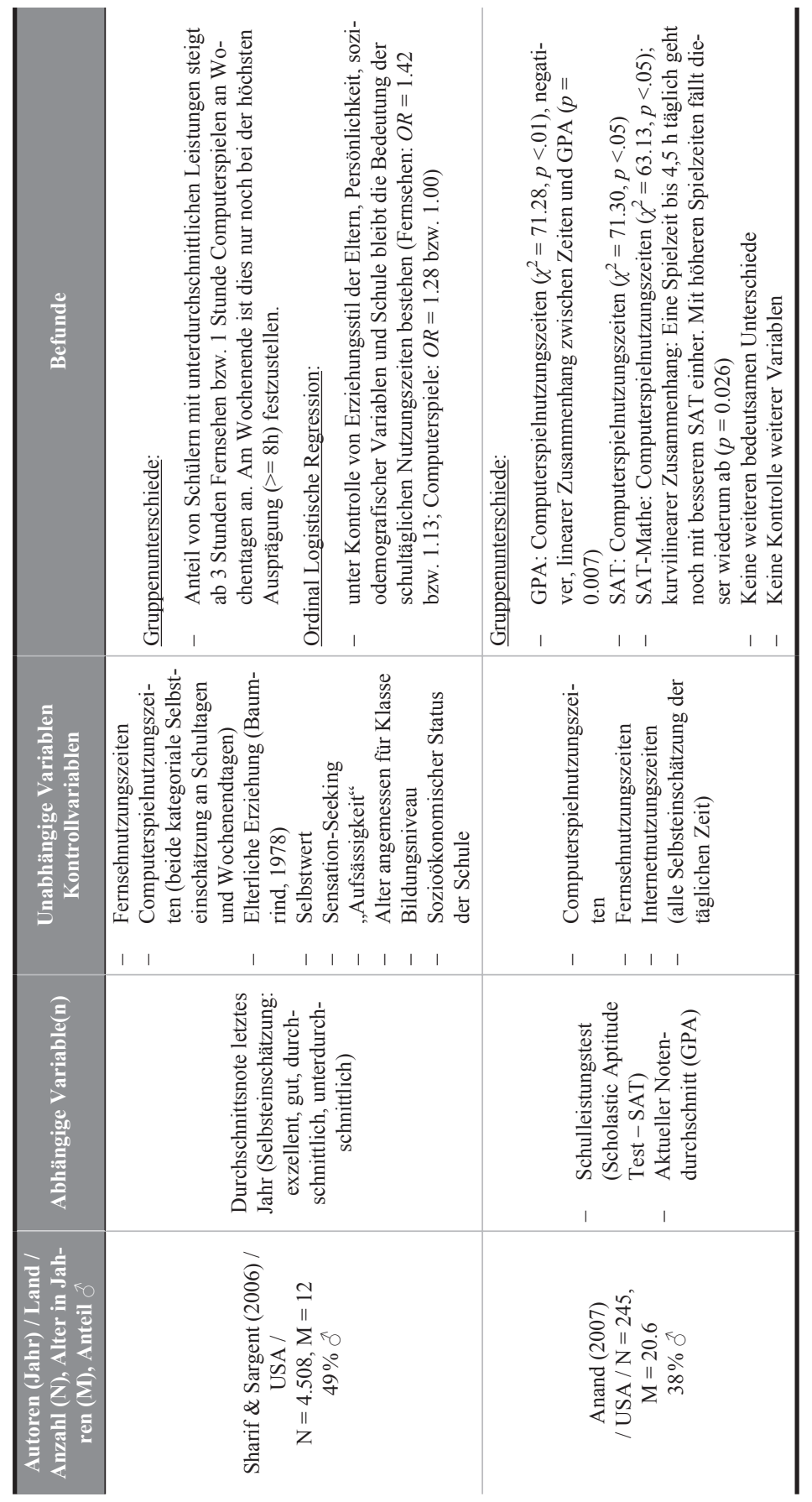




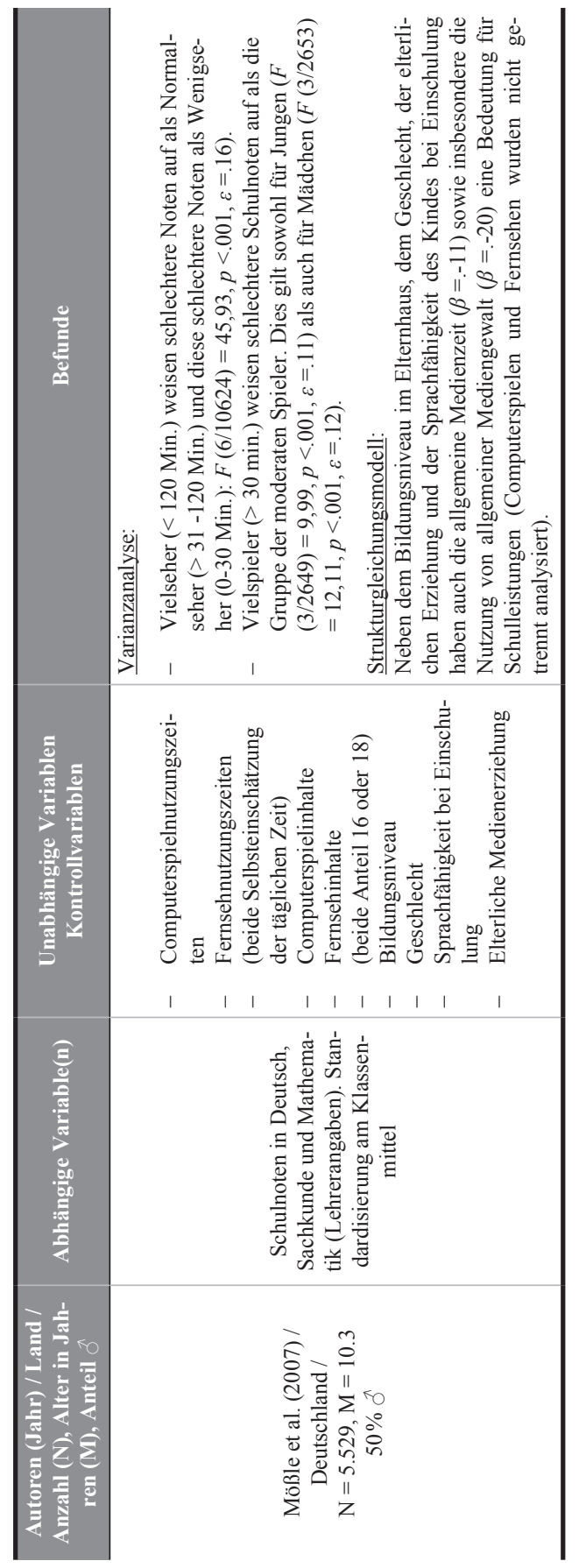




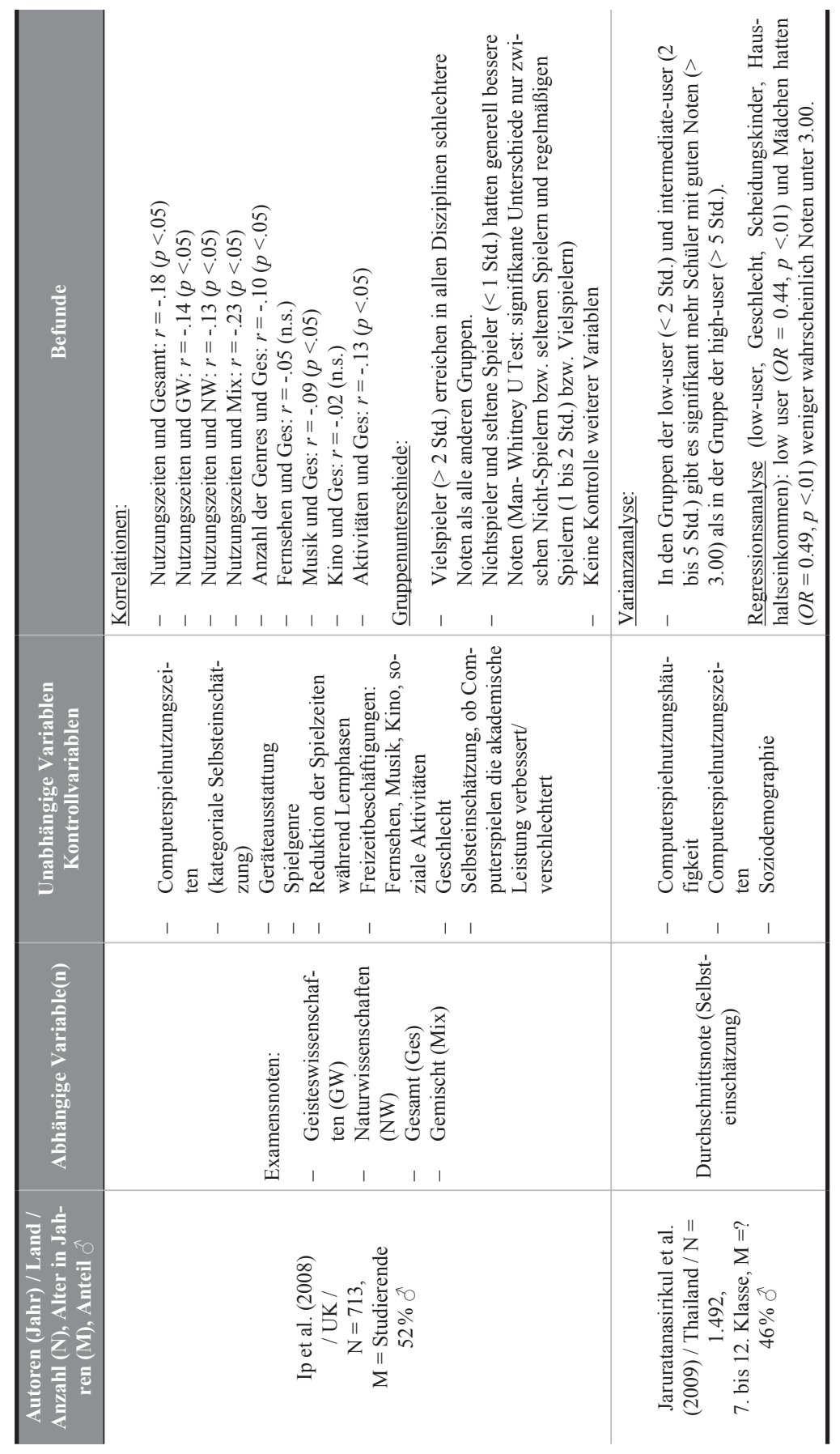




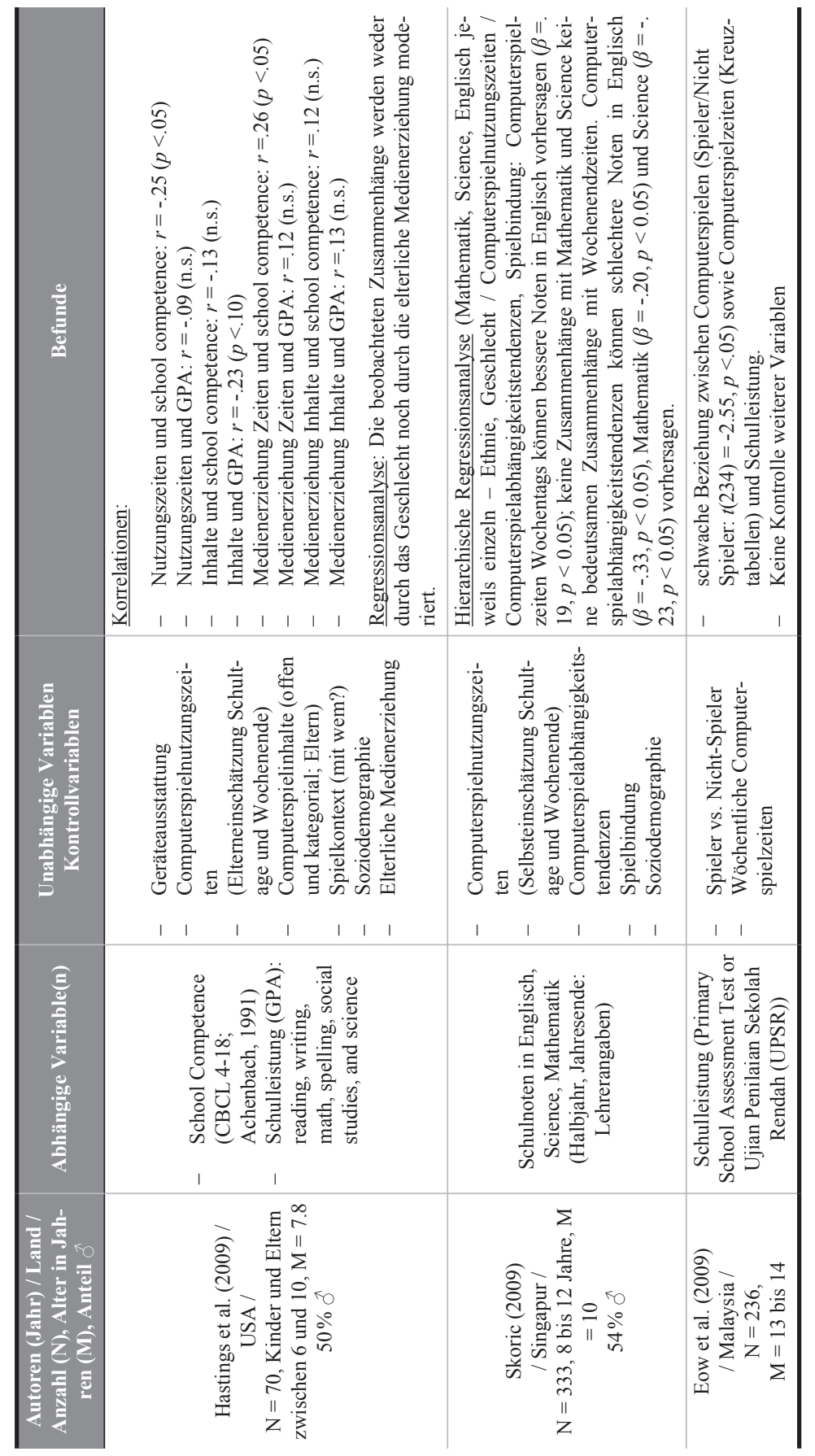




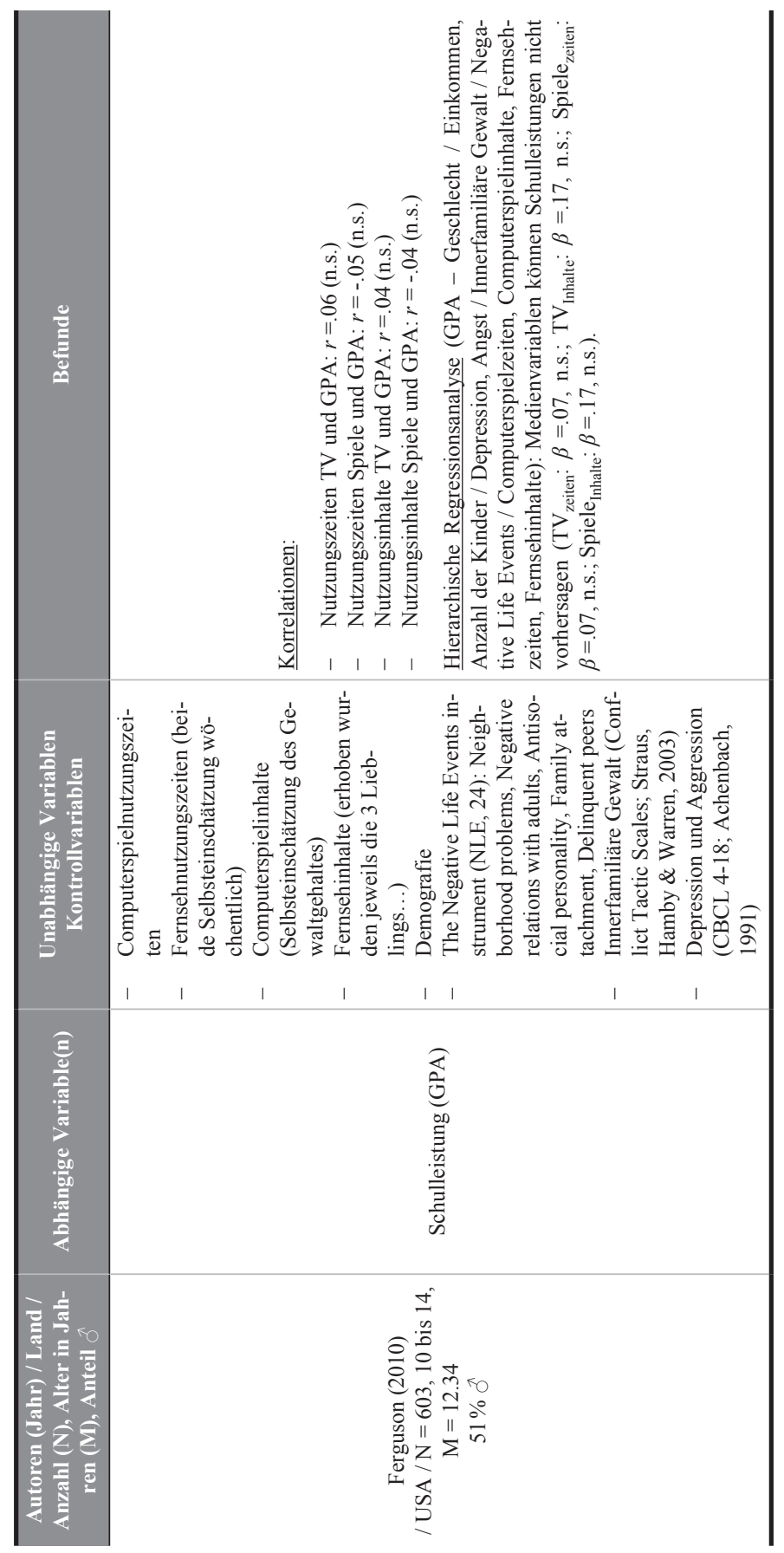




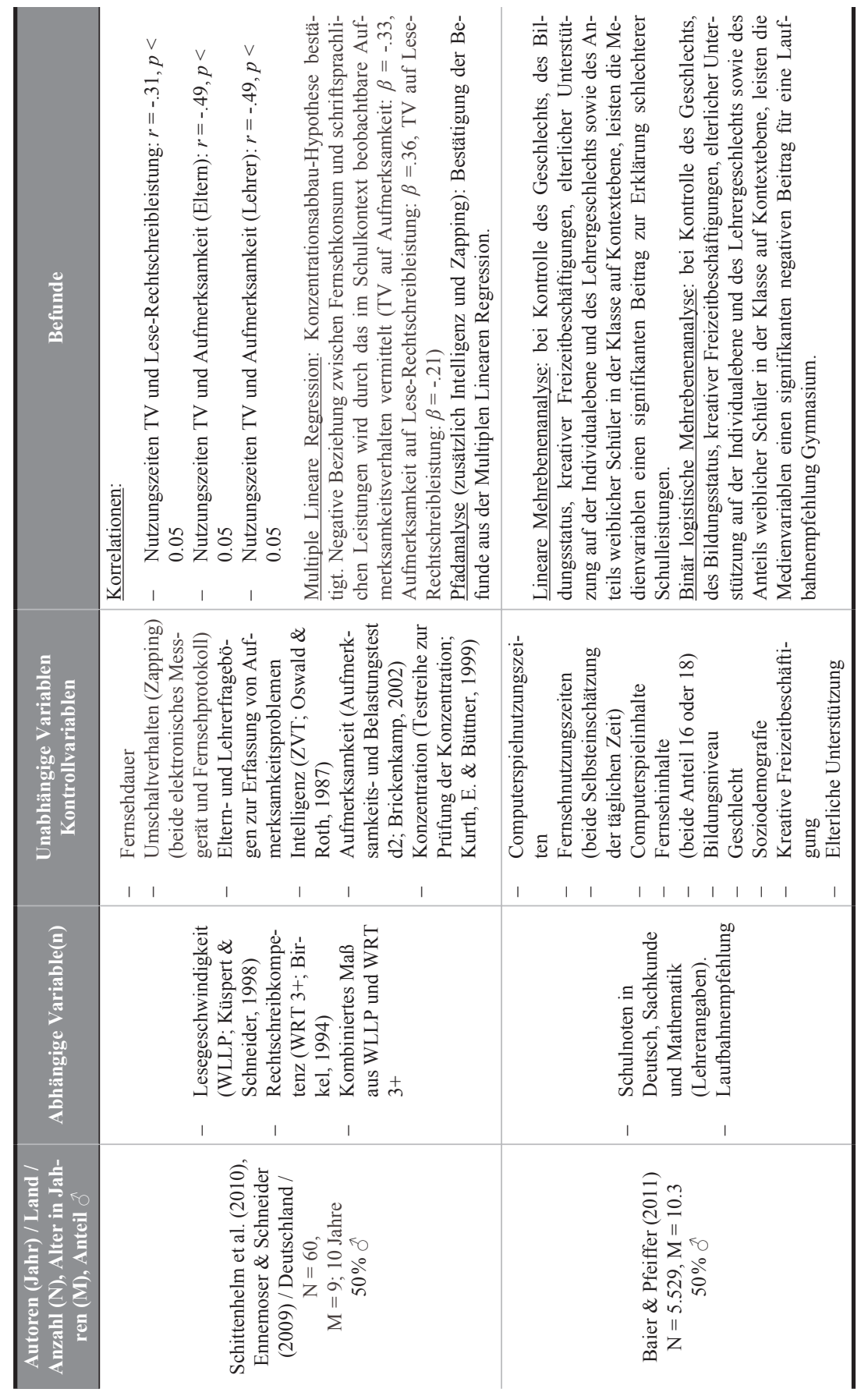




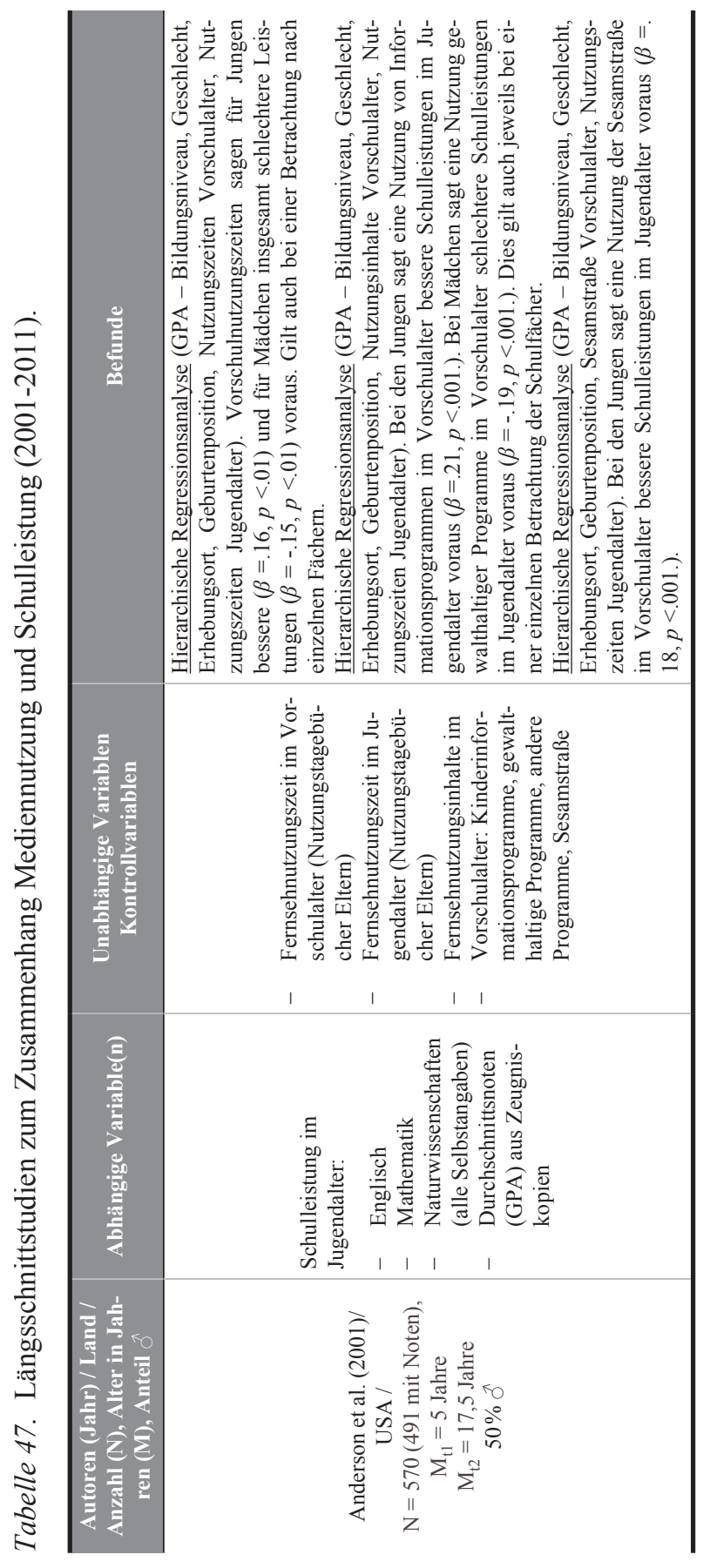




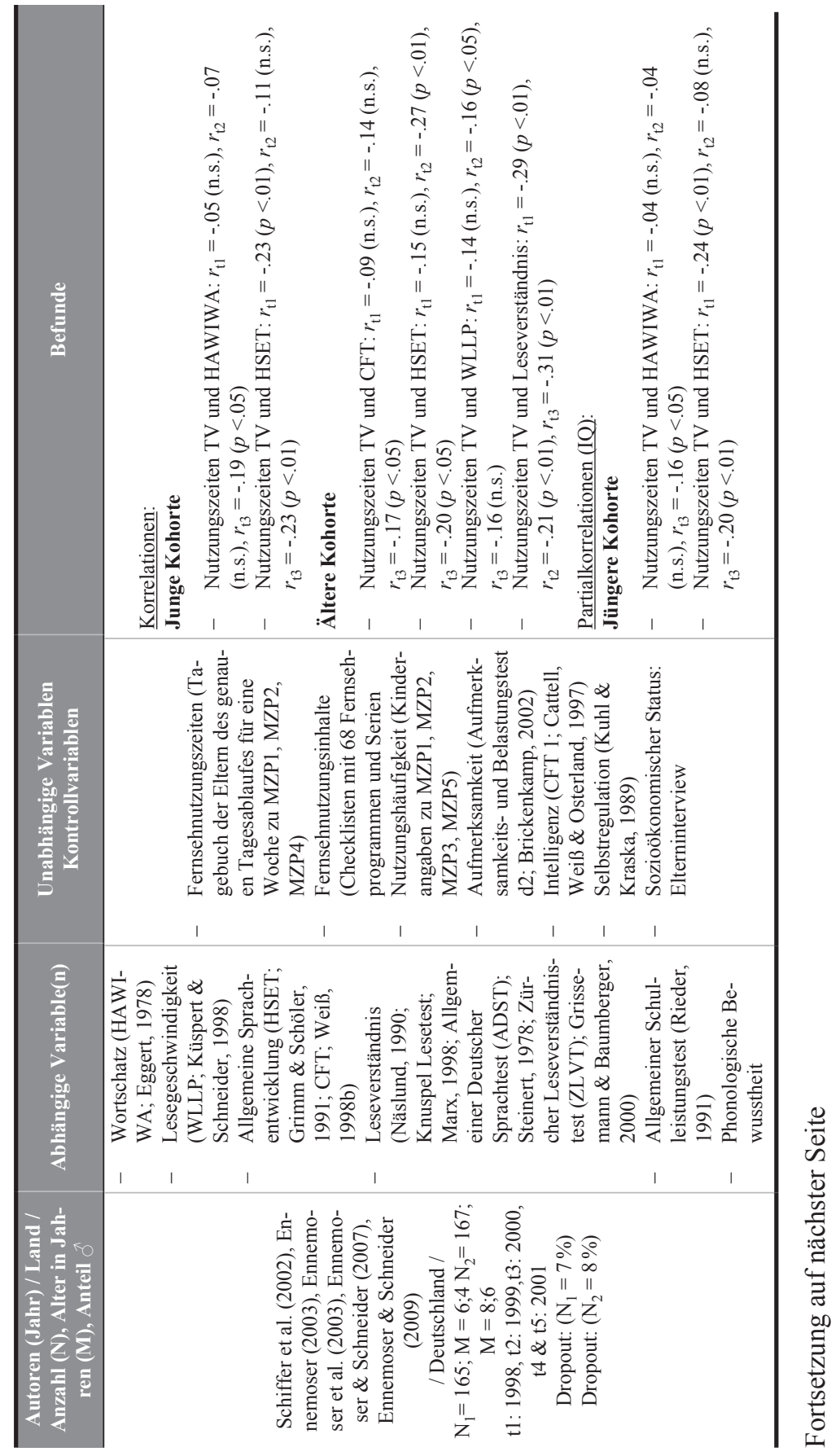




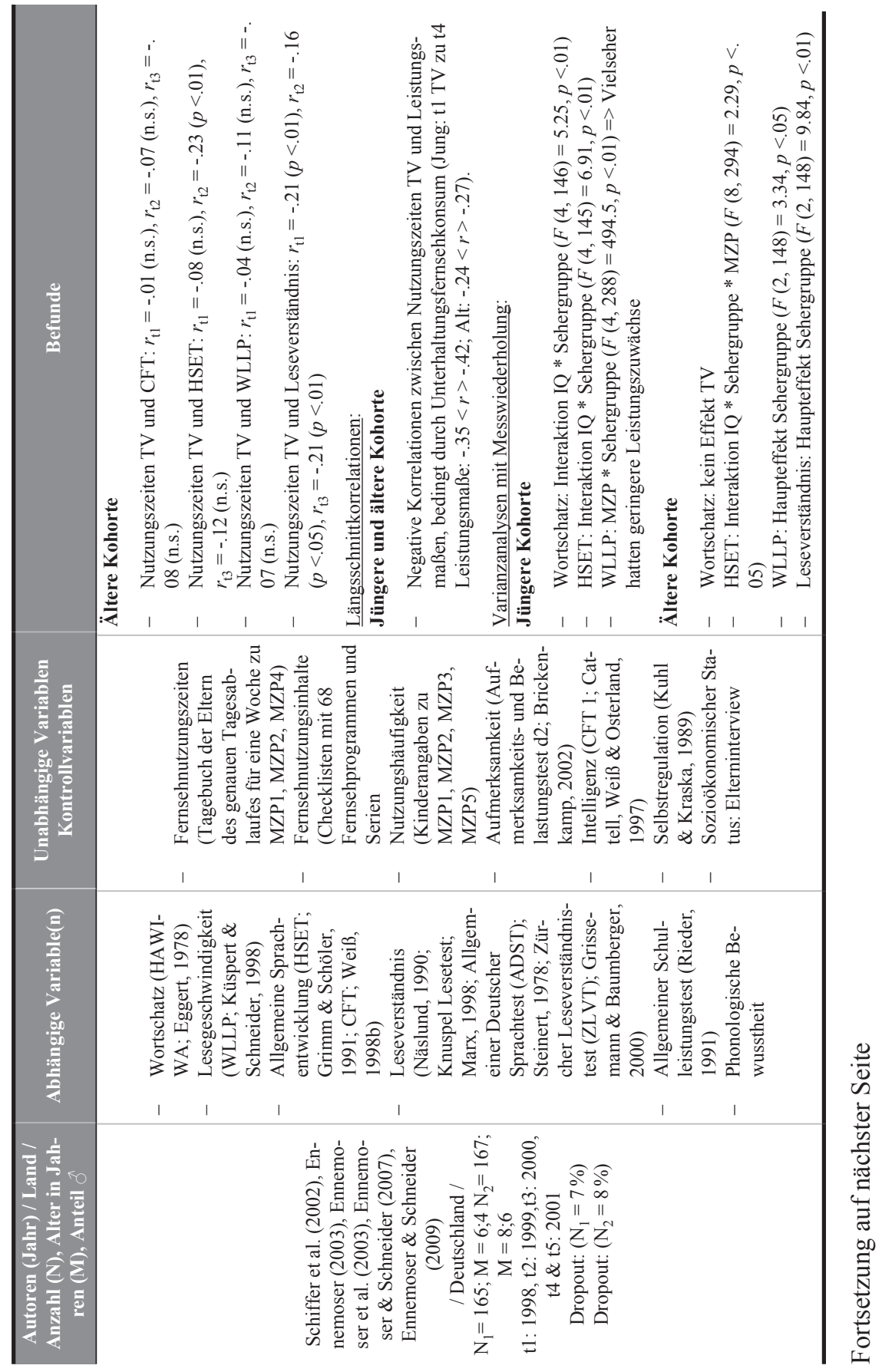




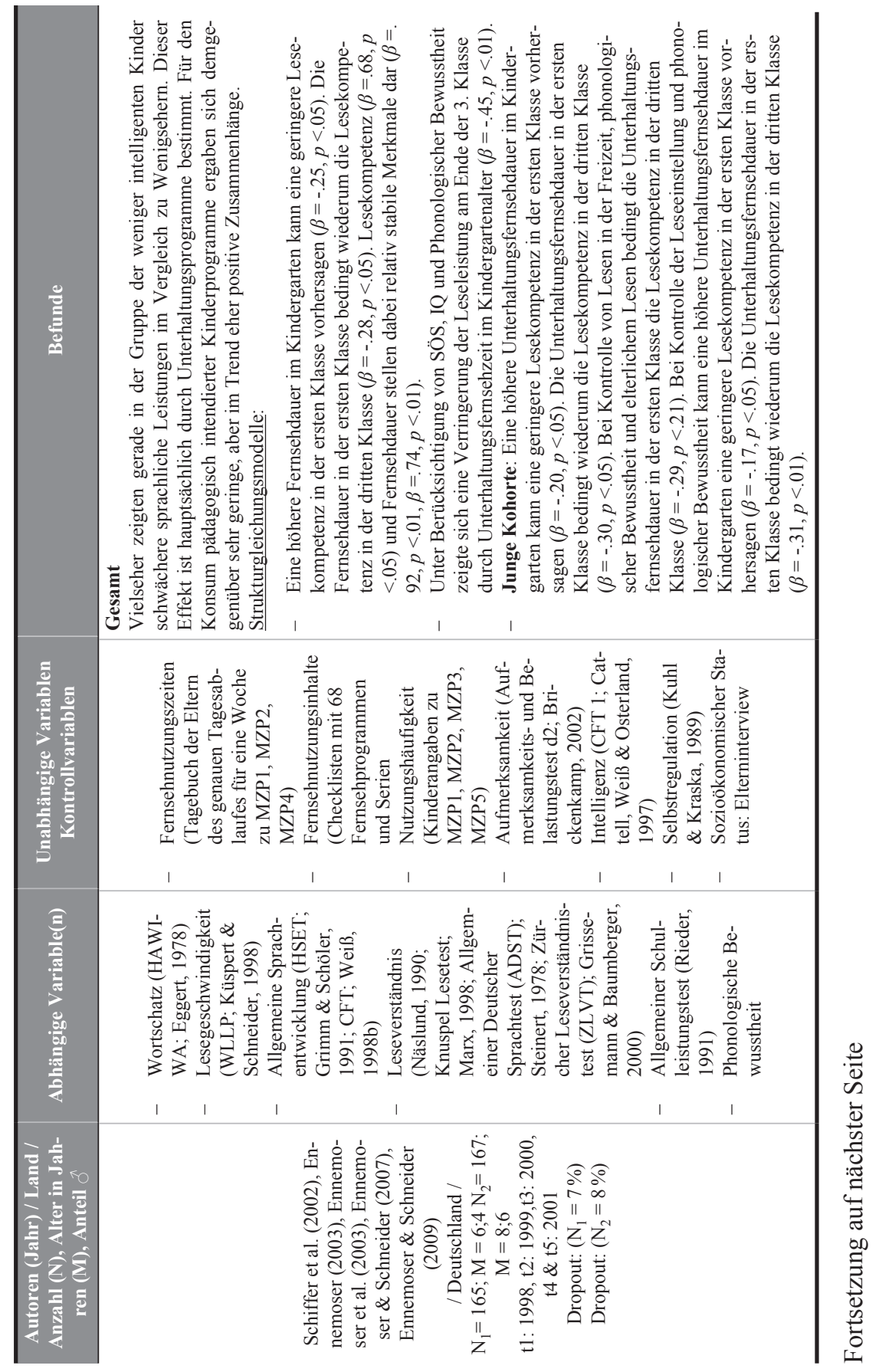




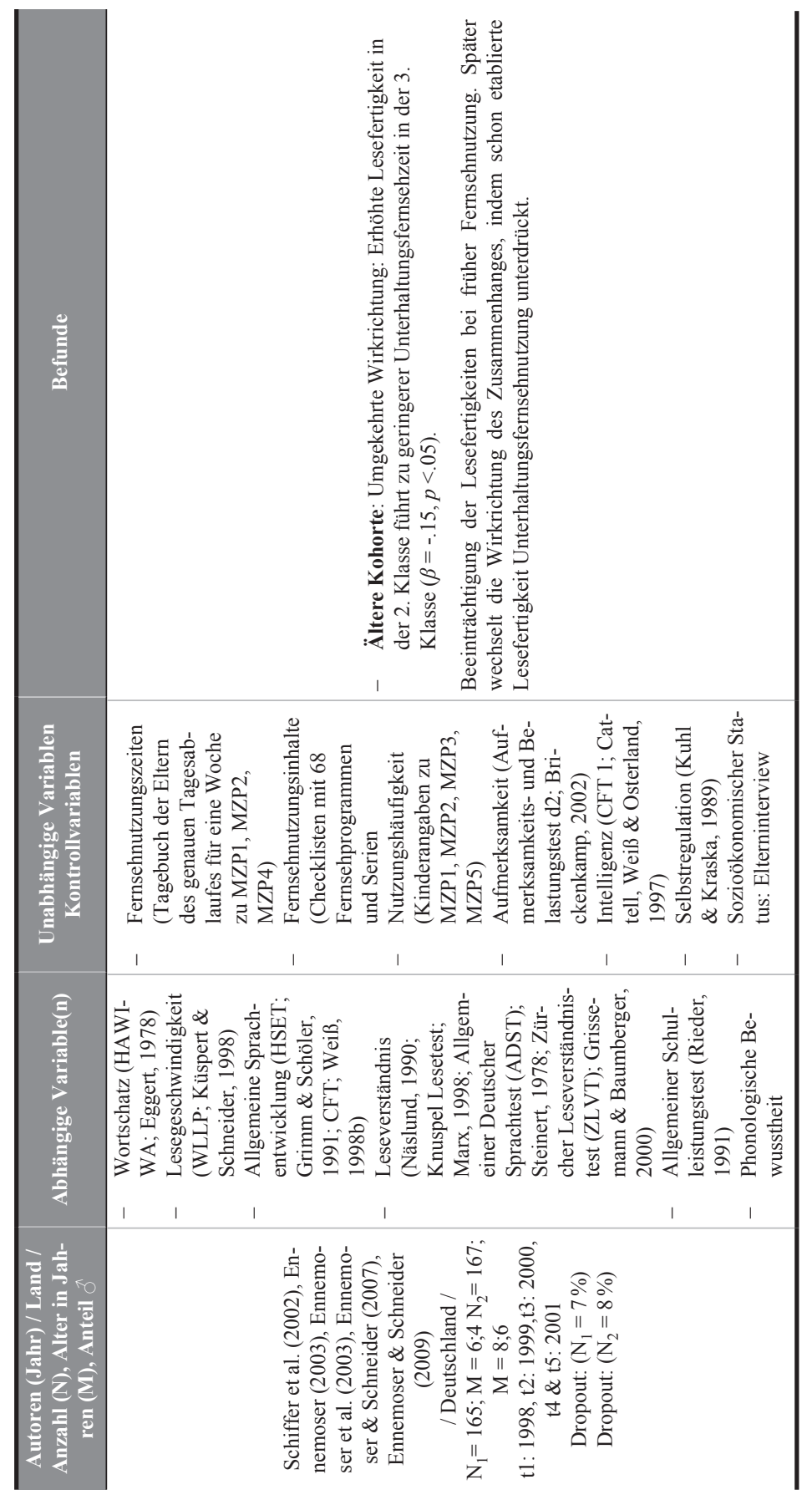




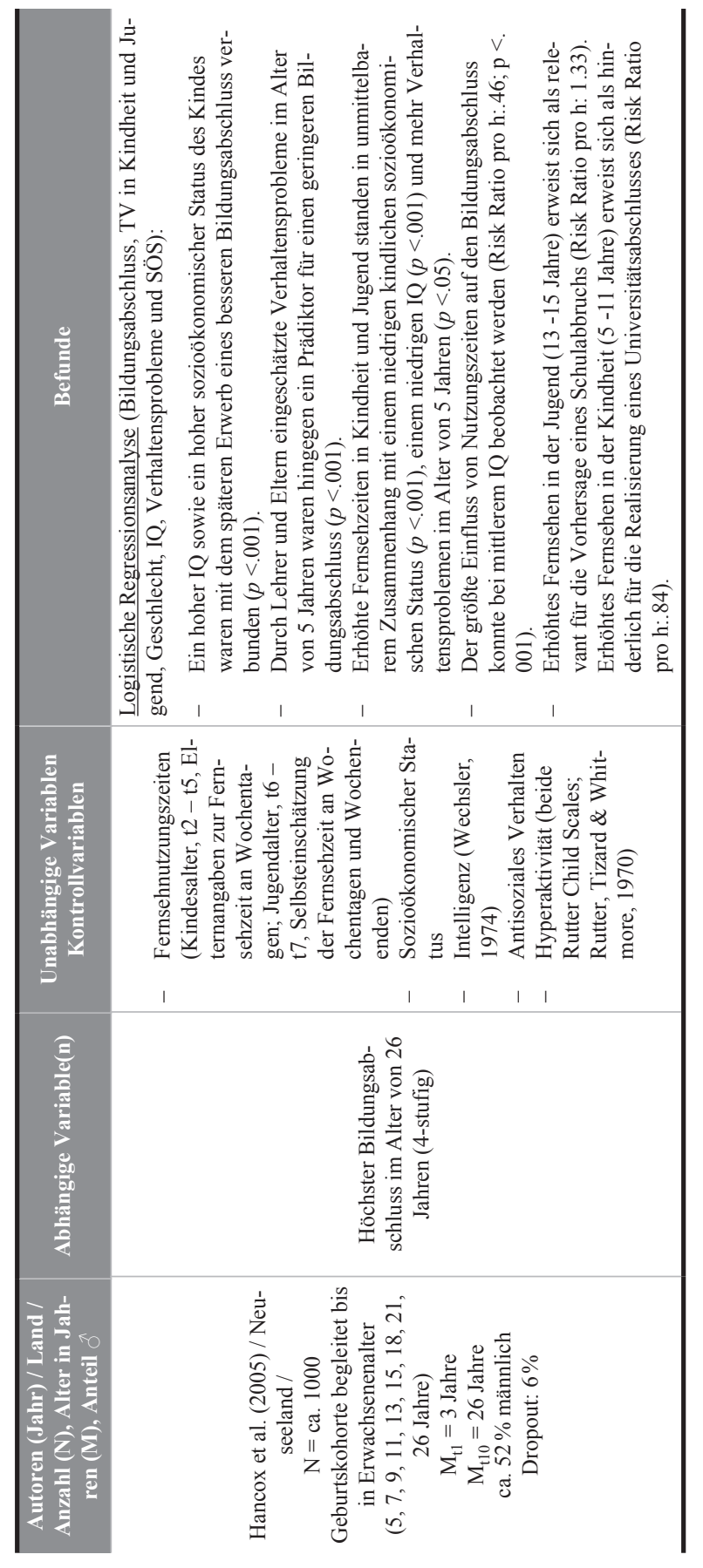




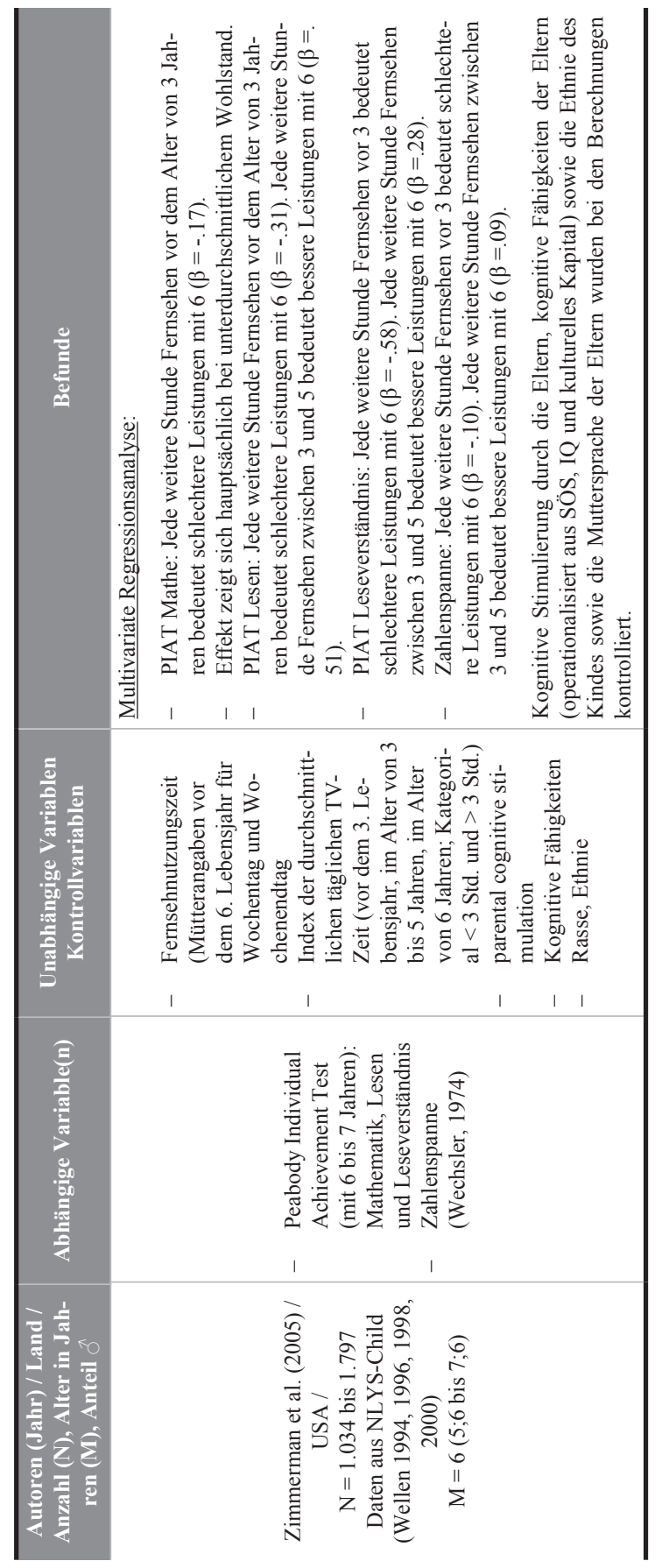




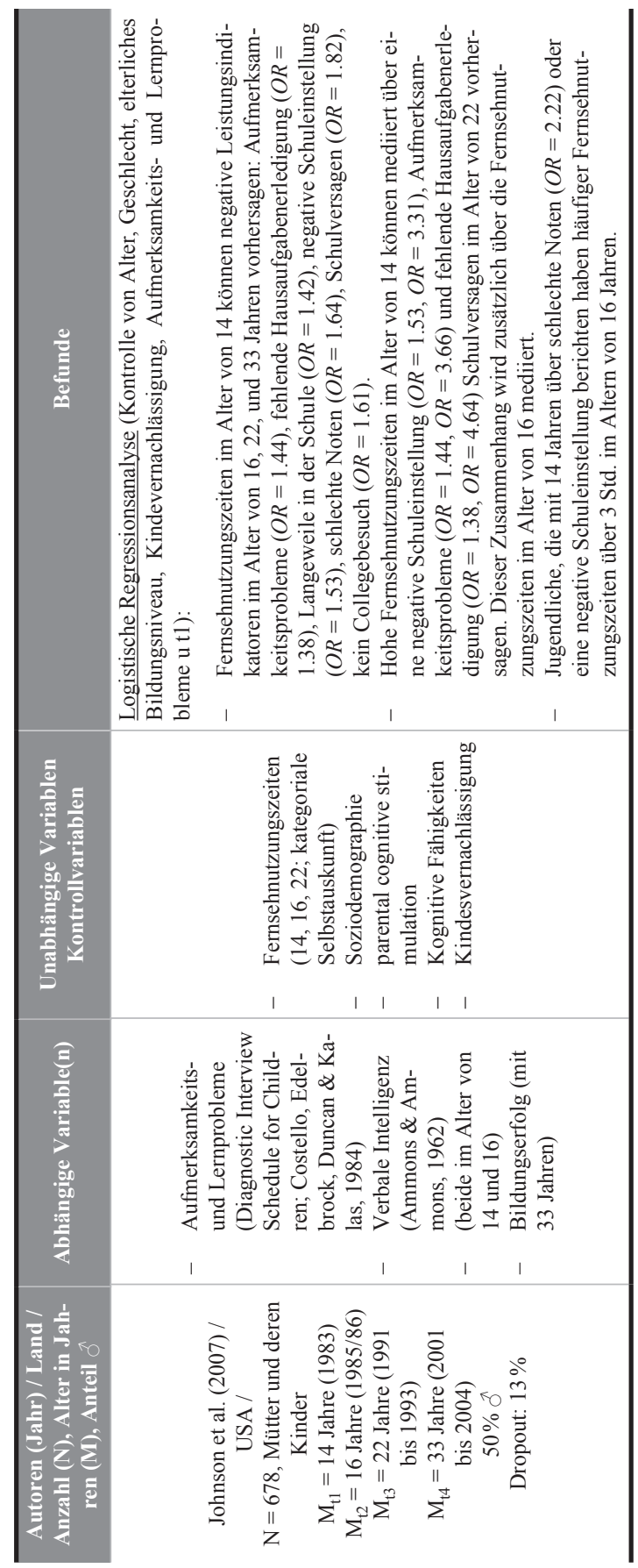




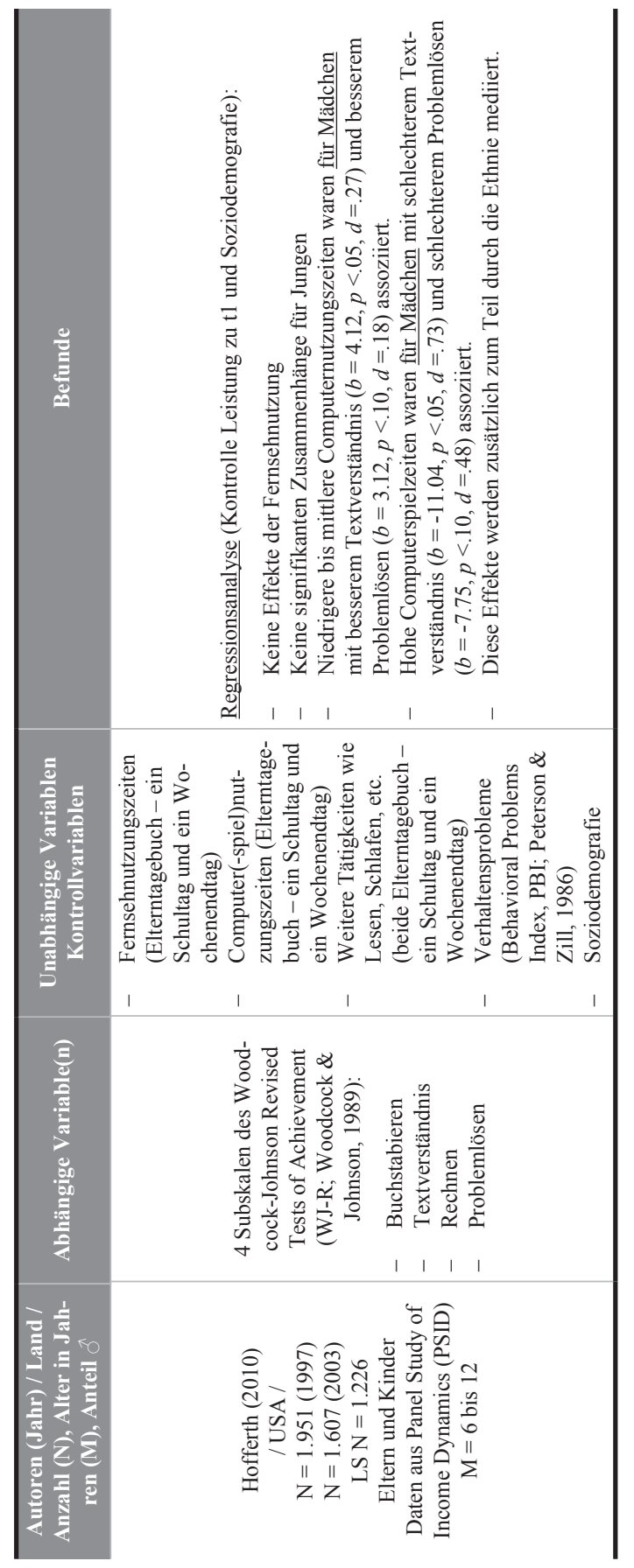




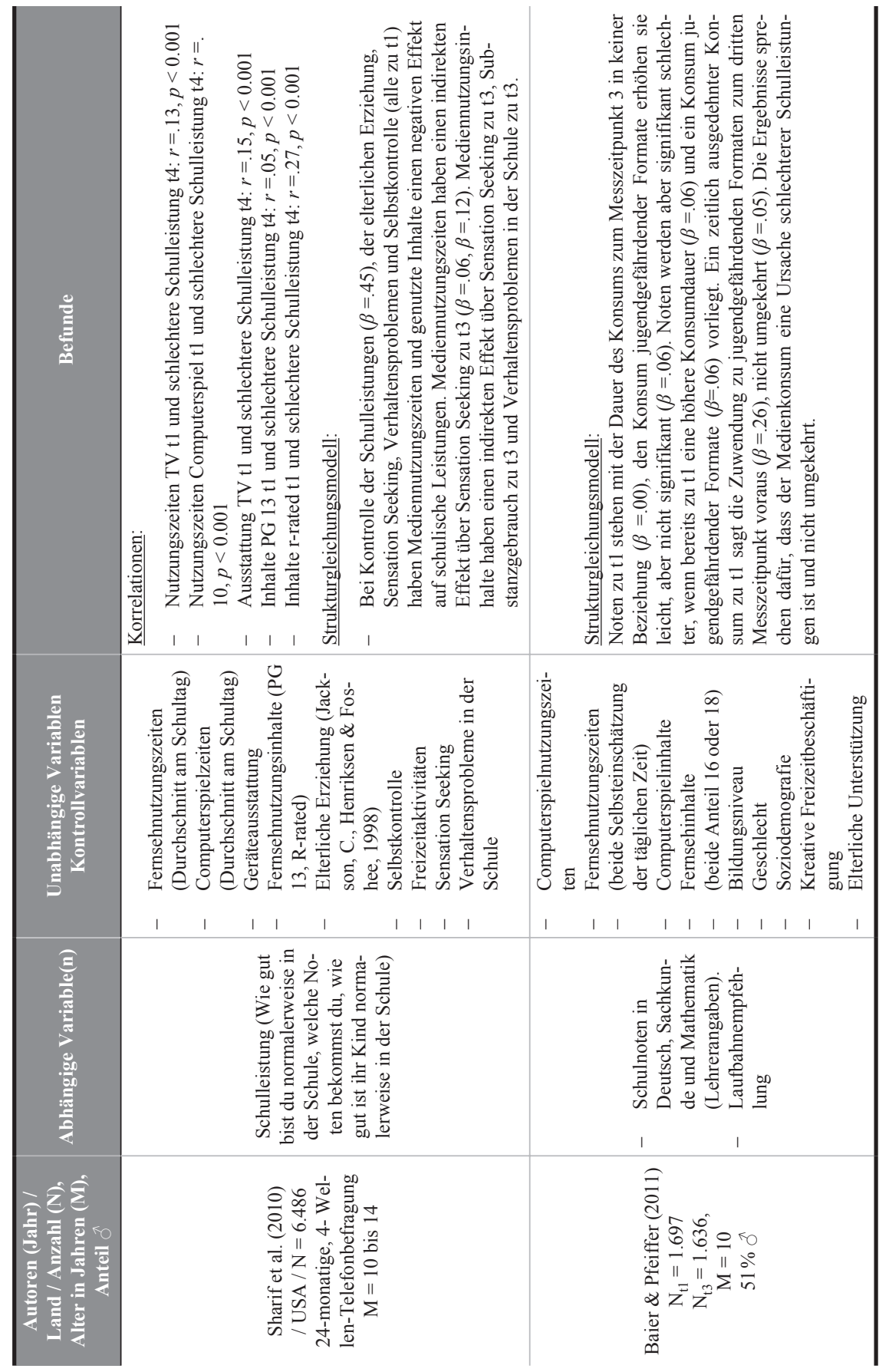




\subsubsection{Erklärungsmodelle zum Zusammenhang Mediennutzung und Schulleistung}

„Zu den wichtigsten Medien zählt das Fernsehen, das positive wie negative Auswirkungen auf die kognitive und soziale Entwicklung und speziell auch auf den Lernerfolg haben kann. Ob der Einfluss günstig oder ungünstig ausfällt hängt von der Art der Sendung und dem Alter und dem Entwicklungsstand der Kinder ab" (Schrader \& Helmke, 2008, S. 289).

In Kapitel 3.1.1 konnte anhand der Forschungsdaten der letzten zehn Jahre dargelegt werden, dass sich Schulleistungen von Kindern und Jugendlichen mit steigendem Fernsehkonsum oder steigendem Computerspielkonsum verschlechtern. Zugleich fanden sich auch Belege dafür, dass den konsumierten Medieninhalten am Fernseher sowie am PC oder der Spielkonsole eine eigenständige Bedeutung zur Erklärung schlechterer Schulleistungen zukommt. Von entscheidendem Interesse ist nun die Frage, wie diese beobachteten Zusammenhänge erklärt werden können.

Zum Wirkzusammenhang von Mediennutzung und Indikatoren schulischer Leistung gibt es unterschiedliche Annahmen, die überblicksartig beispielsweise bei Morgan und Gross (1982), Beentjes und Van der Voort (1988), Anderson, D. R., Huston, Schmitt, Linebarger und Wright (2001) bei Shin (2004) sowie bei Mößle, Rehbein und Kleimann (2007) oder Ennemoser und Schneider (2007) zusammengefasst sind. Grob lassen sich die unterschiedlichen Hypothesen dabei in Stimulierungshypothesen (facilitation hypotheses) und Minderungshypothesen (inhibition hypotheses) unterteilen (vgl. Shin, 2004). Gemäß der Stimulierungshypothesen wird die kognitive Entwicklung von Kindern und Jugendlichen dadurch gefördert, dass z. B. gut gestaltete pädagogische Fernsehprogramme unmittelbar Wissen und Lernstrategien vermitteln, welche in Folge schulische Leistungen verbessern können (zum Beispiel Sprachlernen in der Sesamstraße; vgl. hierzu Anderson, D. R. et al., 2001; Comstock \& Scharrer, 1999; Ennemoser \& Schneider, 2007) oder auch nur weiteres Interesse, wie das Lesen eines Buchs, bei den Kindern und Jugendlichen erzeugen (vgl. Koolstra et al., 1997; Comstock \& Scharrer, 1999; Ennemoser \& Schneider, 2007). Hauptcharakteristikum der Minderungshypothesen ist dagegen, dass Mediennutzung schulische Leistungen negativ beeinflusst, entweder durch die direkte Verminderung der intellektuellen Fähigkeiten der Kinder und Jugendlichen, oder dadurch, dass die Zeit von anderen leistungsförderlichen Tätigkeiten absorbiert wird oder dadurch, dass sie zu einem spezifischem Verhalten führen kann, welches den Schulerfolg von Kindern in der Folge beeinträchtigt (vgl. Shin, 2004; Valkenburg \& van der Voort, 1994). 
Unter den Minderungshypothesen wird der negative Zusammenhang zwischen elektronischer Bildschirmmediennutzung und Schulleistung unter anderem mit verschiedenen Spielarten der Zeitverdrängungshypothese, der wohl bekanntesten Minderungshypothese, erklärt. Gemäß der Hypothese der Zeitverdrängung mindert zeitlich exzessive Mediennutzung einerseits direkt schulischen Erfolg, indem schulbezogene Aufgaben zugunsten der Mediennutzung vernachlässigt werden, und andererseits indirekt, indem andere, leistungsförderliche Freizeitaktivitäten wie Schlaf- und Erholungszeiten verdrängt werden (vgl. Neuman, 1988; Comstock \& Scharrer, 1999; Gentile et al., 2004; Cummings \& Vandewater, 2007; Ennemoser \& Schneider, 2007; Mößle et al., 2007). So leidet beispielsweise auch die körperliche Bewegung, wenn die nachmittäglichen Freizeitaktivitäten hauptsächlich am Bildschirm stattfinden (für eine Metaanalyse siehe Marshall, Biddle, Gorely, Cameron \& Murdey, 2004), wobei die stimulierenden Effekte sportlicher Betätigung oder körperlicher Bewegung auf die kognitive Entwicklung ausbleiben (Ratey, 2009; Kubesch, 2002, 2004; Melillo \& Leisman, 2004). Ein vielseitiges Freizeitverhalten vermittelt demgegenüber wichtige Lernerfahrungen, den Erwerb sozialer Kompetenzen und körperliche sowie psychische Gesundheit und kann deshalb als wichtige Quelle schulischer und beruflicher Erfolge gelten (vgl. Anderson, D. R. et al., 2001; Gentile et al., 2004). Darüber hinaus werden neben Schlafentzug und Bewegungsarmut auch klinische Folgen einer intensiven Mediennutzung im Sinne eines Suchtpotentials, insbesondere der Computerspiele, diskutiert (vgl. Kapitel 5), welche ebenso zu Leistungseinbußen führen können (vgl. Gentile et al., 2004; Gentile et al., 2011; Gentile, 2009; Mößle et al., 2007; Rehbein et al., 2010).

Auf der anderen Seite rückt die Aggressionshypothese bzw. Inhaltshypothese die konsumierten Medieninhalte in den Fokus des Interesses und bezieht Annahmen zu negativen Effekten von Gewaltmediennutzung auf das Sozialverhalten und Aggressivität bzw. Impulsivität von Kindern und Jugendlichen explizit in die Erklärung schlechterer schulischer Leistungen mit ein (vgl. Comstock \& Scharrer, 1999; Anderson, D. R. et al., 2001). Letztere seien immer auch Endprodukt eines sozialen Interaktionsprozesses zwischen Schülerinnen und Schülern (Leistungserbringung) und der Lehrkraft (Leistungsbewertung) und somit auch vom Verhältnis beider Partner beeinflusst (Anderson, D. R. et al., 2001). Nach Anderson, D.R. und Kollegen (2001) kann Aggression bzw. auffälliges Sozialverhalten zu einer Spirale von Ablehnung durch die Lehrkraft, weniger Leistung, geringerer Arbeitsqualität, schlechteren Noten, verringerter Erfolgsmotivation und somit zu einem negativen Kreislauf von Schulversagen, einer Entfremdung von der Lern- und Erfolgskultur und den Zielen, die durch die Schule repräsentiert werden, führen (vgl. MacBeth, 1996; Williams, T. M., 1986; Mößle et al., 2007). 
Vereint werden die Annahmen schulleistungsmindernder Effekte der Zeitverdrängung sowie des Konsums spezifischer (insbesondere gewalthaltiger) Medieninhalte in einer dritten sehr breiten Kategorie der Minderungshypothesen, den sog. Interferenzhypothesen (vgl. Comstock \& Scharrer, 1999). Grundgedanke ist dabei, dass die Nutzung digitaler Bildschirmmedien aufgrund spezifischer Präsentationsformate einerseits zu einer motivationalen Beeinträchtigung (Hypothese der Lesebeeinträchtigung) oder andererseits zu einer direkten Beeinträchtigung der Informationsverarbeitung (Passivitätshypothese, Gedächtnisdefizithypothese, Löschungshypothese) beim Rezipienten führen kann und in Folge dessen die schulische Leistungsfähigkeit gemindert wird. Nach Comstock und Scharrer (1999) kann hier aber auch eine Leistungsminderung durch eine parallel zu Hausaufgaben und Lernen erfolgende Medienrezeption untergeordnet werden, welche in der Forschung zum Teil besser belegt werden konnte als eine reine Zeitverdrängung (vgl. Beentjes \& Van der Voort, 1988; Gaddy, 1986; Armstrong \& Greenberg, 1990).

Die Hypothese der Lesebeeinträchtigung kann kurz dahingehend zusammengefasst werden, dass Kinder durch die angenehmen Erfahrungen, welche sie in der Unterhaltungsmediennutzung (insbesondere des Fernsehens) recht einfach erleben, die Motivation zur Beschäftigung im Rahmen des Schul- und Lernkontextes, wie zum Beispiel dem Lesen, verlieren (vgl. Ennemoser \& Schneider, 2007; siehe auch Beentjes \& Van der Voort, 1988; Koolstra et al., 1997). Eine Leistungsminderung in der Schule, welche sich noch stark an einer textbasierten Vermittlung von Lerninhalten orientiert, würde somit über eine negative Einstellung zum Lesen erklärt werden (vgl. Comstock \& Scharrer, 1999; Koolstra \& Van der Voort, 1996).

In eine ähnliche Richtung argumentiert die Hypothese der Passivität, die davon ausgeht, dass neben der Motivation (siehe Hypothese der Lesebeeinträchtigung) auch Informationsverarbeitungsprozesse durch die geringe geistige Anstrengung zum Konsum von Unterhaltungsmedien (insbesondere des Fernsehens) beeinflusst werden, da diese auch auf das Schreib- und Leselernen übertragen werden könnte (vgl. Ennemoser \& Schneider, 2007; siehe auch Koolstra et al., 1997; Shin, 2004).

Die Informationsverarbeitungsprozesse direkt in den Mittelpunkt rückt die Annahme einer Beeinträchtigung der Konzentrationsfähigkeit durch Medienkonsum. Demnach könnte die Überstimulierung durch rasante Fernsehprogramme mit raschen Kontextwechseln die Fähigkeit von Kindern, sich auf eine bestimmte Aufgabe zu konzentrieren beeinträchtigen (vgl. Ennemoser \& Schneider, 2007; siehe auch Gadberry, 1980; Christakis, Zimmerman, DiGiuseppe \& McCarty, 2004) oder zumindest eine oberflächliche Verarbeitung anregen (Shin, 2004). Maßgeblich hierfür sei, dass durch eine fixierte Aufmerksamkeitszuwendung, 
wie sie bei der Mediennutzung beobachtet werden kann, eine selektive Aufmerksamkeitszuwendung wenig gefördert wird, was in Folge auch zu einer weniger zielgerichteten Zuwendungsfähigkeit zu schulischen Reizen, welche zudem deutlich weniger rasant sind und eine geringe Reizdichte mit sich bringen, führen kann (vgl. Ennemoser \& Schneider, 2007; Christakis et al., 2004). Eine empirische Bestätigung dieser Annahme konnte bis jetzt allerdings noch nicht erbracht werden; eine Erklärung über motivationale Prozesse (Hypothese der Passivität, Hypothese der Lesebeeinträchtigung) scheint wahrscheinlicher als eine dauerhafte Minderung der Konzentrationsfähigkeit (vgl. Ennemoser \& Schneider, 2007; Rehbein, 2011).

Waren letztere Hypothesen vor allem auf der Grundlage der Fernsehwirkungsforschung entstanden, bezieht eine letzte Interferenzhypothese, die der Löschung gelernter Inhalte, auch Wirkannahmen zu Computerspielen mit ein. So stützt sich diese auf Erkenntnisse gedächtnispsychologischer und neurobiologischer Forschung, die darauf hinweisen, dass sowohl mediale Gewaltdarstellungen als auch die besonderen biophysiologischen Erregungsmuster bei der Nutzung gewalthaltiger Medien einen unmittelbaren Einfluss auf die Informationsverarbeitungprozesse des Nutzers nehmen können, und geht davon aus, dass die Erinnerung des in der Schule Gelernten durch den Medienkonsum beeinträchtigt ist (vgl. Spitzer, 2005; Scheich, 2006). So sind der Anblick und das Miterleben emotionalisierender Bilder, insbesondere von Gewaltbildern, in aller Regel mit starken Gefühlszuständen verbunden (vgl. auch Koepp et al., 1998, die eine um 100 Prozent erhöhte Ausschüttung von Dopamin beim Spielen von Gewaltspielen feststellen konnten). Die Rezeption emotional geladener Medieninhalte (z. B. aus Horrorfilmen oder gewalthaltigen Computerspielen) geht mit einer Erhöhung physiologischer Stressparameter einher wie Herzschlag, Blutdruck, Adrenalin, Cortisol und Noradrenalin (vgl. Anderson, C. A., Gentile \& Buckley, 2007; Baldaro et al., 2004; Carnagey, Anderson \& Bushman, 2007; Skosnik, Chatterton, Swisher \& Park, 2000). Durch eine solche Aktivierung der Stressachse kann eine Beeinträchtigung vorangegangener Lernvorgänge ausgelöst werden (McGaugh, 2000; Cahill \& McGaugh, 1996). Die Auswirkungen auf das Lernen sind jedoch immer eine Frage der Stressdosis: Ein moderates emotionales Erleben bzw. moderater Stress wirken durchaus anregend und können die Qualität vorangegangener Lernvorgänge sogar steigern (McGaugh, 2000; Cahill \& McGaugh, 1996). Erst ab einer gewissen Stärke wirkt sich die emotionale Erregung negativ auf zuvor akquirierte Gedächtnisinhalte aus. Die Gefahr einer solchen Lernbeeinträchtigung besteht umso stärker, je kürzer die Lernerfahrung zurückliegt, da über den Hippocampus vermittelte deklarative Gedächtnisinhalte noch weniger stark konsolidiert sind (Izquierdo \& Medina, 1997). 
„Es ist offensichtlich, dass Medienkonsum mit stark emotional wirksamen Inhalten eine bevorzugte Verankerung solcher Informationen bewirkt. Dies geschieht in Konkurrenz zu weniger aufwühlenden Erfahrungen z. B. im Schulalltag. [...] Die Verankerung einer Information im Langzeitgedächtnis dauert mehr als 24 Stunden. Wird während dieser Zeit die Information wiederholt oder durch ähnliche Informationen variiert, verstärkt sich die Verankerung im Langzeitgedächtnis. Dies erklärt einerseits den massiven Effekt von regelmäßigem Langzeitkonsum bestimmter Medien, andererseits aber auch den Sinn nachmittäglicher Hausaufgaben in der Schule oder Ganztagsschule mit Stoffvertiefungen am Nachmittag. Nimmt TV-Konsum oder Computerspielen die zeitlich und emotional dominierende Rolle im Tagesverlauf ein, hat Schulstoff keine Chance fest im Langzeitgedächtnis verankert zu werden.“ (Scheich, 2006, S. 277f)

In der Forschung konnte diese Hypothese der Löschung spezifischer Gedächtnisinhalte bisher allerdings noch nicht bestätigt werden (vgl. Rehbein, 2011).

Insgesamt scheinen sowohl Hypothesen der Zeitverdrängung als auch Inhaltsund Interferenzhypothesen ihre Berechtigung zur Erklärung schulleistungsmindernder Effekte von Unterhaltungsmediennutzung zu besitzen. Eine Verknüpfung der unterschiedlichen Hypothesen, wie sie bereits die Kategorie der Interferenzhypothesen nahe legt, scheint aber vor dem Hintergrund der multifaktoriellen Determiniertheit schulischer Leistung (vgl. Abbildung 48) nicht nur sinnvoll, sondern notwendig.

\subsection{Ergebnisse des Berliner Längsschnitt Medien - Mediennutzung und Schulleistung}

In Kapitel 3.1 konnten zahlreiche Belege aus nationalen wie internationalen Studien der letzten zehn Jahre dafür aufgeführt werden, dass sowohl der Dauer als auch den konsumierten Inhalten des täglichen Medienkonsums in Bezug auf die Entwicklung schulischer Leistungen eine große Bedeutung zukommt. Ein Schwerpunkt bei der Konzipierung des Berliner Längsschnitts Medien war die Aufklärung dieses Zusammenhangs unter Berücksichtigung weiterer Determinanten häuslicher Mediennutzung sowie schulischer Leistung. Hierzu wurden neben den sozial-strukturellen Merkmalen Migrationsstatus, Wohlstand und Bildungshintergrund im Elternhaus, die sich nicht nur unmittelbar auf die Mediennutzungsgewohnheiten der Kinder auswirken, sondern auch direkt mit deren Schulleistungen in Zusammenhang stehen, auch die kognitiven Fähigkeiten der Kinder, gemessen mit dem CFT 20, deren Schuleinstellung und Selbstkonzept eigener Schulfähigkeiten sowie das allgemeine elterliche Erziehungs- und Unterstützungsverhalten berücksichtigt (zur Variablenbildung siehe Kapitel 1.5). 
In der KFN-Schülerbefragung 2005 (Mößle et al., 2007) hatten z. B. Kinder der vierten Klasse mit einem eigenen Fernseher im Zimmer deutlich schlechtere Schulnoten in den Fächern Deutsch, Mathematik und Sachkunde. Der gleiche Zusammenhang ließ sich beobachten, wenn man die Kinder nach Nutzungszeiten aufteilte: Schülerinnen und Schüler mit sehr hohen Mediennutzungszeiten schnitten deutlich schlechter in der Schule ab als ihre Altersgenossen mit geringerer Mediennutzungszeit. Noch deutlichere Auswirkungen auf die Schulleistungen waren in der KFN-Schülerbefragung 2005 zu beobachten, wenn nach den konsumierten Inhalten unterschieden wurde. Je öfter die Kinder der vierten Klasse nicht altersadäquate Spiele (USK 16, USK 18) spielten, desto schlechter waren ihre Schulnoten. Als wichtigste Einflussgrößen auf den kindlichen Medienkonsum zeigten sich in dieser Querschnittsbefragung eine gewaltfreie Erziehung, der Bildungshintergrund im Elternhaus sowie eine aktive Medienerziehung der Eltern. Letztere wurden bereits im obigen Strukturgleichungsmodell zum Mediennutzungsverhalten der Kinder direkt in Beziehung gesetzt (vgl. Abbildung 47).

Von entscheidender Bedeutung für den Berliner Längsschnitt Medien sind nun zwei Fragen: Lassen sich erstens die in der KFN-Schülerbefragung 2005 sowie in weiteren nationalen wie internationalen (quer- wie längsschnittlichen) Studien berichteten Zusammenhänge zwischen einer inhaltlich problematischen sowie einer zeitlich exzessiven Mediennutzung und schlechteren Schulleistungen bei Berücksichtigung weiterer aus der Literatur bekannter Einflussvariablen auch für die Berliner Grundschülerpopulation bestätigen (Kapitel 3.2.2, 3.2.3)? Können zweitens Befunde hinsichtlich einer Wirkungsvermutung (problematische Mediennutzung führt zu schlechteren Schulleistungen) im Gegensatz zu einer Selektion (schlechtere Schülerinnen und Schüler nutzen vermehrt Medien) im Längsschnitt bestätigt werden (Kapitel 3.2.4)?

Da sich Jungen und Mädchen in ihrer Mediennutzung deutlich unterscheiden und Jungen bereits in der dritten Klasse deutlich länger elektronische Medien nutzen und sehr viel stärkere Präferenzen für gewalttätige Inhalte ausweisen (vgl. Kapitel 2 - Unterschiede, die auch für eine Unterscheidung nach Bildungsniveau, Migrationshintergrund, Wohlstand oder elterlichem Erziehungsverhalten berichtet werden könnten), soll im Folgenden untersucht werden, inwieweit solche Mediennutzungsmuster mit schlechteren schulischen Leistungen assoziiert sind und wie sich ein solcher Zusammenhang interpretieren lässt.

Bevor diese Zusammenhänge in Kapitel 3.2.3 und 3.2.4 vertieft werden, soll jedoch zunächst die allgemeine Entwicklung der Schulnoten im Berliner Längsschnitt Medien (Kapitel 3.2.1) mit ihren bivariaten Zusammenhängen zu unterschiedlichen sozial-strukturellen wie Medienvariablen (Kapitel 3.2.2) beschrieben werden. 


\subsubsection{Entwicklung schulischer Leistung im Grundschulalter}

Vom ersten Befragungszeitpunkt im November 2005 an wurden zu allen fünf durchgeführten Messzeitpunkten im Lehrerfragebogen die Schulnoten des letzten Zeugnisses sowie standardisierte Schulleistungen des aktuellen Leistungsstandes in den Kernfächern Deutsch, Mathematik, Sachkunde sowie Sport erhoben. Die Noten im Fach Sachkunde wurden in der dritten und vierten Klasse, die Noten im Fach Englisch in der fünften und sechsten Klasse abgefragt. Dargestellt in Tabelle 48 sind aus Gründen der Vergleichbarkeit jeweils nur die Noten gemessen am Ende der dritten, vierten, fünften und sechsten Klasse, da diese später mit den Mediennutzungszeiten in Beziehung gesetzt werden sollen.

Tabelle 48. Mittlere Noten nach Messzeitpunkt.

\begin{tabular}{|c|c|c|c|c|c|c|c|c|}
\hline & \multicolumn{2}{|c|}{ 3. Klasse } & \multicolumn{2}{|c|}{ 4. Klasse } & \multicolumn{2}{|c|}{ 5. Klasse } & \multicolumn{2}{|c|}{ 6. Klasse } \\
\hline & $N$ & $M(S D)$ & $N$ & $M(S D)$ & $N$ & $M(S D)$ & $N$ & $M(S D)$ \\
\hline Deutsch & 741 & $2.38(.88)$ & 735 & $2.54(.86)$ & 752 & $2.70(.85)$ & 712 & $2.75(.87)$ \\
\hline Jungen & 373 & $2.49(.87)$ & 365 & $2.64(.89)$ & 380 & $2.86(.85)$ & 357 & $2.90(.86)$ \\
\hline \multirow[t]{2}{*}{ Mädchen } & 368 & $2.27(.87)$ & 370 & $2.45(.82)$ & 372 & $2.54(.82)$ & 355 & $2.61(.85)$ \\
\hline & & $r=-.13 * *$ & & $r=-.11 * *$ & & $r=-.18^{* *}$ & & $r=-.17 * *$ \\
\hline Mathematik & 742 & $2.43(.95)$ & 736 & $2.65(.93)$ & 751 & $2.76(.93)$ & 711 & $2.82(1.0)$ \\
\hline Jungen & 373 & $2.32(.91)$ & 367 & $2.57(.92)$ & 380 & $2.71(.94)$ & 357 & $2.80(1.0)$ \\
\hline \multirow[t]{2}{*}{ Mädchen } & 369 & $2.54(.97)$ & 369 & $2.72(.94)$ & 371 & $2.81(.91)$ & 354 & $2.84(1.0)$ \\
\hline & & $r=.11^{*}$ & & $r=.08^{*}$ & & $r=.05^{\mathrm{ns}}$ & & $r=.02^{\mathrm{ns}}$ \\
\hline Sachkunde & 742 & $2.28(.86)$ & 735 & $2.39(.89)$ & & & & \\
\hline Jungen & 373 & $2.33(.89)$ & 366 & $2.45(.96)$ & & & & \\
\hline \multirow[t]{2}{*}{ Mädchen } & 369 & $2.22(.83)$ & 639 & $2.33(.81)$ & & & & \\
\hline & & $r=-.06^{i}$ & & $r=-.07^{\imath}$ & & & & \\
\hline Englisch & & & & & 753 & $2.55(.98)$ & 711 & $2.70(1.0)$ \\
\hline Jungen & & & & & 381 & $2.66(1.0)$ & 355 & $2.84(1.0)$ \\
\hline \multirow[t]{2}{*}{ Mädchen } & & & & & 372 & $2.45(.92)$ & 356 & $2.55(.99)$ \\
\hline & & & & & & $r=-.11 * *$ & & $r=-.14 * *$ \\
\hline Sport & 731 & $2.03(.77)$ & 736 & $2.19(.77)$ & 751 & $2.13(.78)$ & 708 & $2.07(.79)$ \\
\hline Jungen & 368 & $2.00(.80)$ & 367 & $2.22(.79)$ & 380 & $2.13(.81)$ & 354 & $2.08(.80)$ \\
\hline \multirow[t]{2}{*}{ Mädchen } & 363 & $2.06(.74)$ & 369 & $2.16(.75)$ & 371 & $2.13(.75)$ & 354 & $2.06(.79)$ \\
\hline & & $r=.03^{\mathrm{ns}}$ & & $r=-.04^{\mathrm{ns}}$ & & $r=.01^{\mathrm{ns}}$ & & $r=-.01^{\mathrm{ns}}$ \\
\hline
\end{tabular}

Anmerkung. ${ }^{*} p<.05,{ }^{* *} p<.01, \imath p<.10, n s=$ nicht signifikant. Korrelationen nach Pearson.

Insgesamt verschlechtern sich die mittleren Noten in allen erhobenen Fächern (mit Ausnahme von Sport) von der dritten bis zur sechsten Klasse. Dies ist zum einen auf eine mit höherem Schuljahr stärkere Leistungsdifferenzierung, speziell 
im unteren Leistungsbereich, zurückzuführen. So wurden in der dritten Klasse die Noten fünf und sechs sowie mitunter auch vier noch relativ selten vergeben. ${ }^{91}$ Zum anderen ist insbesondere bei den berichteten Noten der fünften und sechsten Klasse der Wegfall einiger besonders leistungsstarker Schüler, die auf eine weiterführende Schule gewechselt haben, zu berücksichtigen.

Geschlecht. Hinsichtlich der mittleren Deutschnote ist bezüglich des Geschlechts ein klarer Vorsprung der Mädchen gegenüber den Jungen zu beobachten, der sich von der dritten bis zur sechsten Klasse zusätzlich vergrößert. So wird auch der bestehende korrelative Zusammenhang zwischen Geschlecht und Schulnoten - statistisch allerdings nicht bedeutsam $(z=-0,77)$ - über die Jahre stärker. ${ }^{92}$ Betrachtet man die mittleren Mathematiknoten, sind zu Beginn der dritten Klasse die Jungen noch klar besser als die Mädchen. Dieser Unterschied nivelliert sich bis zur sechsten Klasse jedoch fast, da die Jungen sich in stärkerem Maße verschlechtern als die Mädchen. ${ }^{93}$ In Sachkunde bleibt der relativ geringe (und nicht bedeutsame) Vorsprung der Mädchen in den mittleren Noten von der dritten in die vierte Klasse bestehen. ${ }^{94}$ In Englisch sind, gemäß der Annahme einer besseren Sprachbegabung der Mädchen, die mittleren Noten der Mädchen besser als die der Jungen. Auch hier verschlechtern sich die Jungen nach einem Jahr in einem stärkeren Ausmaß als die Mädchen. ${ }^{95}$ In Sport sind hingegen keine Geschlechterunterschiede sowie insgesamt kaum Veränderungen über die Jahre zu beobachten. ${ }^{96}$

91 In den meisten Klassen wurden zudem erst ab der dritten Klasse Noten für die Kinder vergeben, was eine geringere Differenzierung zum Teil erklärt.

922 (Geschlecht) x 4 (Klasse) ANOVA mit Messwiederholung auf dem Faktor Deutschnote: zwei statistisch bedeutsame Haupteffekte, Geschlecht $F(1 / 441)=11.54, p<.001, f=.16$ und Zeit $F(2,81 / 1323)=39.89, p<.001, f=.30$, sowie eine statistisch bedeutsame Interaktion Zeit x Geschlecht $F(2,81 / 1323)=6.90, p<.001, f=.12$.

932 (Geschlecht) x 4 (Klasse) ANOVA mit Messwiederholung auf dem Faktor Mathematiknote: statistisch bedeutsamer Haupteffekt Zeit $F(2,88 / 1317)=40.27, p<.001, f=.30$ sowie eine statistisch bedeutsame Interaktion Zeit x Geschlecht $F(2,88 / 1317)=4.00, p<$. $001, f=.09$; Geschlecht $F(1 / 439)=3.49, p=.06, f=.09$.

942 (Geschlecht) x 2 (Klasse) ANOVA mit Messwiederholung auf dem Faktor Sachkundenote: statistisch bedeutsamer Haupteffekt Zeit $F(1 / 638)=21.17, p<.001, f=.18$; Geschlecht $F(1 / 638)=2.50, p=.11, f=.06$.

952 (Geschlecht) x 4 (Klasse) ANOVA mit Messwiederholung auf dem Faktor Englischnote: zwei statistisch bedeutsame Haupteffekte, Geschlecht $F(1 / 648)=10.96, p<.001, f=.13$ und Zeit $F(1 / 648)=33.14, p<.001, f=.23$, sowie eine statistisch bedeutsame Interaktion Zeit x Geschlecht $F(1 / 648)=9.01, p<.01, f=.12$.

962 (Geschlecht) x 4 (Klasse) ANOVA mit Messwiederholung auf dem Faktor Sportnote: statistisch bedeutsamer Haupteffekte Zeit $F(2.80 / 1308)=5.36, p<.01, f=.11$. 


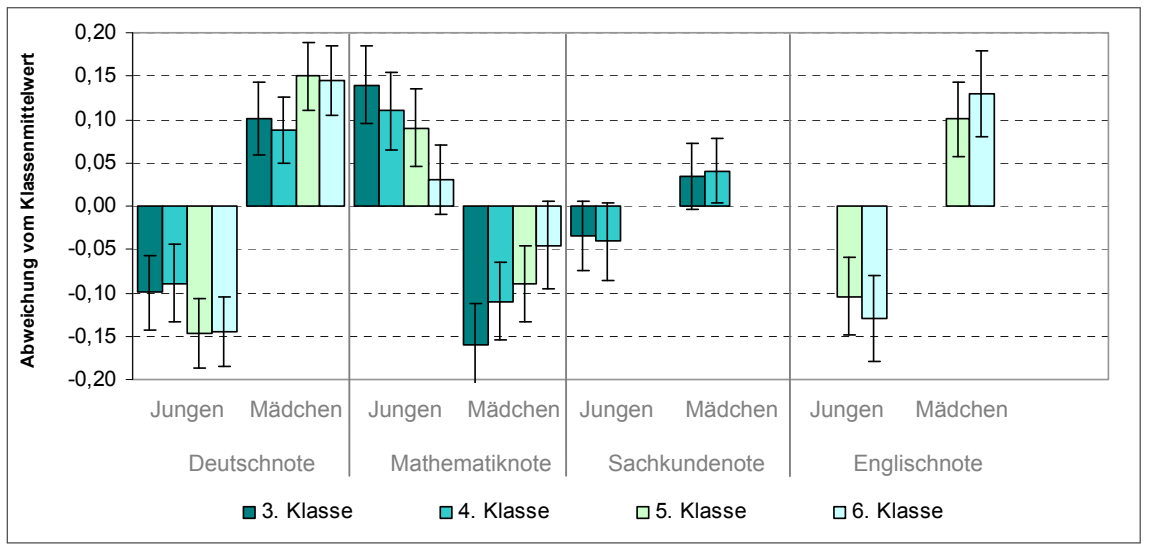

Abbildung 50. Notenabweichungen von Klassenmittewert nach Fach, Geschlecht und Messzeitpunkt. Fehlerbalken zeigen Standardfehler des Mittelwerts.

In Abbildung 50 wurden, um unterschiedlichen Benotungsstilen oder einer bestimmten Bewertungskultur in den jeweiligen Klassen Rechnung zu tragen, alle Noten am jeweiligen Klassenmittelwert in diesem Fach relativiert. Eine Abweichung nach oben steht somit für eine im Vergleich zum Klassendurchschnitt bessere, eine Abweichung nach unten für eine im Vergleich zum Klassendurchschnitt schlechtere Leistung. Auch hier zeigt sich noch einmal recht anschaulich der klare Vorsprung der Mädchen gegenüber den Jungen im Fach Deutsch, der sich von der dritten bis zur fünften Klasse zudem vergrößert. Darüber hinaus wird auch offensichtlich, dass sich der am Ende der dritten Klasse beobachtete Vorsprung der Jungen im Fach Mathematik bis zur fünften Klasse annähernd nivelliert.

\subsubsection{Bedingungsfaktoren schulischer Leistung - ein Rahmenmodell}

In den Schwerpunktauswertungen der KFN-Schülerbefragung 2005 konnten für 10-jährige Grundschülerinnen und Grundschüler neben dem Bildungsniveau im Elternhaus auch die Präferenz für Mediengewaltinhalte sowie die täglichen Mediennutzungszeiten zur Erklärung bestehender Schulleistungsunterschiede identifiziert werden (vgl. Mößle et al., 2007). Weitere aus der Literatur bekannte Einflussvariablen auf Seiten der Schülerinnen und Schülern, die mit Schulleistungen assoziiert sind, konnten jedoch aufgrund des Studiendesigns nicht berücksichtigt werden. Um die Gültigkeit des beobachteten Beziehungsgeflechts unter Berück- 
sichtigung weiterer (Persönlichkeits-)Merkmale überprüfen zu können, wurden neben dem Geschlecht der Kinder die folgenden Variablen berücksichtigt: Zur Erfassung des sozialen und kulturellen Kapitals der Migrationshintergrund, das Bildungsniveau im Elternhaus sowie der familiäre Wohlstand (zur Berechnung siehe alle Kapitel 1.4.1), auf Seiten des Schülers oder der Schülerin die Schuleinstellung, ${ }^{97}$ das Selbstkonzept eigener Schulfähigkeiten ${ }^{98}$ sowie die kognitiven Fähigkeiten, gemessen mit dem Grundintelligenztest Skala 2 (CFT 20, 1. Teil; Weiß, 1998b) und auf Seiten der Eltern das elterliche Erziehungs- und Unterstützungsverhalten. ${ }^{99}$

Um einen ersten Eindruck zur differenziellen Bedeutung der einzelnen Variablen für die Erklärung bestehender Schulleistungsunterschiede zu bekommen, sind in Tabelle 49 und Tabelle 50 Mittelwertsunterschiede sowie Querschnittskorrelationen getrennt nach den unterschiedlichen Variablen, Messzeitpunkten und Fächern dargestellt.

Migrationshintergrund. Bezüglich der mittleren Deutsch-, Mathematik- und Sportnote ist hinsichtlich des Migrationshintergrundes der Kinder am Ende der dritten Klasse ein klarer Vorsprung der Kinder ohne gegenüber den Kindern mit Migrationshintergrund zu beobachten. Ein Unterschied, der sich bis zur sechsten Klasse lediglich für Deutsch und Sport verringert, da sich Kinder ohne Migrationshintergrund in beiden Fächern in stärkerem Maße verschlechtern als Kinder mit Migrationshintergrund. In Mathematik bleibt der Unterschied bestehen. In Sachkunde ist der relativ große Vorsprung der Kinder ohne Migrationshintergrund in den mittleren Noten von der dritten in die vierte Klasse konstant. In Englisch sind die mittleren Noten der Kinder mit Migrationshintergrund in vergleichbarem Maß schlechter.

Bildungsniveau im Elternhaus. Betrachtet man das Bildungsniveau im Elternhaus, sind - zusammen mit einer Differenzierung nach familiärem Wohlstand - die deutlichsten Unterschiede in den mittleren Noten zu konstatieren. Sowohl in Deutsch, Mathematik und Sachkunde als auch in Englisch und Sport sind Kinder aus Familien mit niedrigem Bildungsniveau (höchster Bildungsabschluss beider Elternteile ist der Hauptschulabschluss) deutlich schlechter als Kinder mit mittlerem Bildungsniveau (höchster Bildungsabschluss mindestens eines Elternteils ist die Mittlere Reife), welche wiederum klar schlechter ab-

97 Mittelwerte der drei viertstufigen Items: „Morgens freue ich mich auf die Schule“, „Schule macht Spaß“, „Ich gehe gern zur Schule“. Hohe Werte auf dieser Skala stehen für Freude und Spaß an der Schule (Itemkennwerte siehe Kapitel 1.5.1, Tabelle 17).

98 Skala Selbstkonzept eigener Schulfähigkeiten: Summenscore der vierstufigen (,stimmt nicht“, ,stimmt kaum“, ,stimmt ziemlich“, „stimmt genau“) Items: „Ich lerne sehr langsam (umgepolt)“, „Ich mache in der Schule das meiste richtig“, „Ich kann ganz gut lernen“, „Ich bin gut in der Schule“. Hohe Werte auf dieser Skala stehen für eine hohe Einschätzung ihrer schulischen Fähigkeiten (siehe Kapitel 1.5.2, Tabelle 21).

99 Vgl. Kapitel 1.5.1, siehe Tabelle 14. 
schneiden als Kinder aus Familien mit hohem Bildungsniveau (höchster Bildungsabschluss mindestens eines Elternteils ist das Abitur oder ein abgeschlossenes Studium). Diese Unterschiede vergrößern sich über die Schuljahre in den beiden Kernfächern Deutsch $\left(r_{3 . \text { Klasse }}=-.22 ; r_{6 \text {.Klasse }}=-.34 ; z=-2.36\right)$ und Mathematik $\left(r_{3 . \text { Klasse }}=-.22 ; r_{5 . \text { Klasse }}=-.29 ; z=-1.35\right)$ deutlich bzw. leicht, bleiben konstant in vergleichbarer Größenordnung in Sachkunde und Englisch und verringern sich etwas in Sport. In Englisch sind die Unterschiede ähnlich stark ausgeprägt wie in Mathematik oder Deutsch gemessen zu den vergleichbaren Messzeitpunkten in der fünften und sechsten Klasse.

Wohlstand im Elternhaus. Die Befunde zum Zusammenhang von familiärem Wohlstand und mittleren Schulnoten sind vergleichbar mit denen zum Bildungsniveau im Elternhaus. Sowohl in Deutsch, Mathematik und Sachkunde als auch in Englisch und Sport sind bessere Noten mit steigendem familiärem Wohlstand festzustellen. Über die Jahre vergrößern sich diese Unterschiede wiederum in den beiden Kernfächern Deutsch und Mathematik, sowie in Sachkunde, bleiben konstant in Englisch und reduzieren sich etwas in Sport.

Elterliches Erziehungs- und Unterstützungsverhalten. Es zeigt sich, dass bei einem Großteil der Schülerinnen und Schüler günstige Erziehungsvoraussetzungen mit hoher elterlicher Zuwendung zu beobachten sind (3. Klasse: 62\%, 4. Klasse: $69 \%$, 5. Klasse: $66 \%$, 6. Klasse: $60 \%$ ). Mittlere elterliche Zuwendung findet sich in rund einem Drittel der Haushalte (3. Klasse: 35\%, 4. Klasse: $28 \%$, 5. Klasse: $31 \%$, 6. Klasse: $35 \%$ ). Über geringe elterliche Zuwendung berichten zwischen 2 (3. Klasse) und 5 Prozent (6. Klasse) der Kinder. Zum bivariaten Zusammenhang mit Schulnoten zeigt sich, dass in allen erhobenen Fächern diejenigen Kinder, die über eine geringe elterliche Zuwendung berichten, schlechtere mittlere Noten haben als jene, die das elterliche Erziehungs- und Unterstützungsverhalten als mittelmäßig einschätzen, welche wiederum schlechter abschneiden als Kinder mit hoher elterlicher Zuwendung. Ein Zusammenhang, der je nach Messzeitpunkt und Schulfach stärker oder schwächer ausgeprägt ist (zwischen $r=-.07$ und $r=-.24)$. 
Tabelle 49. Zusammenhänge Schulnoten in Deutsch und Mathematik mit sozialstrukturellen Merkmalen.

\begin{tabular}{|c|c|c|c|c|c|c|c|c|}
\hline & \multicolumn{4}{|c|}{ D } & \multicolumn{4}{|c|}{$\mathbf{M}$} \\
\hline & 3. & 4. & 5. & 6. & 3. & 4. & 5. & 6. \\
\hline & $M(S D)$ & $M(S D)$ & $M(S D)$ & $M(S D)$ & $M(S D)$ & $M(S D)$ & $M(S D)$ & $M(S D)$ \\
\hline \multicolumn{9}{|c|}{ Migrationshintergrund } \\
\hline Deutsch & $2.26(.84)$ & $2.44(.81)$ & $2.63(.84)$ & $2.65(.82)$ & $2.37(.88)$ & $2.55(.89)$ & $2.70(.91)$ & $2.75(1.0)$ \\
\hline $\mathrm{MH}$ & $2.76(.89)$ & $2.85(.94)$ & $2.92(.84)$ & $3.04(.93)$ & $2.62(1.1)$ & 2.95 (.99) & $2.94(.96)$ & $3.01(1.1)$ \\
\hline$r$ & $.24 * *$ & $.20 * *$ & $.15^{* *}$ & $.20 * *$ & $.12 * *$ & $.18 * *$ & $.11 * *$ & $.11 * *$ \\
\hline \multicolumn{9}{|c|}{ Bildungsniveau } \\
\hline niedrig & $2.71(.88)$ & $3.08(.83)$ & $3.24(.75)$ & $3.20(.70)$ & $2.67(1.0)$ & $2.98(.92)$ & $3.17(.93)$ & $3.17(.98)$ \\
\hline mittel & $2.49(.87)$ & $2.64(.79)$ & $2.82(.80)$ & $2.91(.80)$ & $2.60(.93)$ & $2.82(.91)$ & $2.94(.89)$ & $3.03(.99)$ \\
\hline hoch & $2.18(.78)$ & $2.32(.83)$ & $2.39(.75)$ & $2.44(.81)$ & $2.21(.82)$ & $2.44(.88)$ & $2.47(.86)$ & $2.48(.98)$ \\
\hline$r$ & $-.22 * *$ & $-.28 * *$ & $-.36 * *$ & $-.34 * *$ & $-.22 * *$ & $-.24 * *$ & $-.30 * *$ & $-.29 * *$ \\
\hline \multicolumn{9}{|c|}{ Wohlstand } \\
\hline Q1 & $2.73(.86)$ & $2.87(.92)$ & $3.03(.81)$ & $3.16(.84)$ & $2.69(1.1)$ & $3.00(.99)$ & $3.01(.98)$ & $3.12(1.1)$ \\
\hline Q2 & $2.57(.88)$ & $2.67(.79)$ & $2.85(.79)$ & $3.01(.90)$ & $2.62(1.0)$ & $2.80(.93)$ & $2.94(.85)$ & $3.01(1.0)$ \\
\hline Q3 & $2.32(.85)$ & $2.55(.77)$ & $2.75(.83)$ & $2.69(.79)$ & $2.39(.93)$ & $2.69(.88)$ & $2.88(.94)$ & $2.90(.95)$ \\
\hline Q4 & $2.16(.88)$ & $2.39(.81)$ & $2.56(.86)$ & $2.55(.81)$ & $2.27(.82)$ & $2.41(.86)$ & $2.59(.87)$ & $2.68(1.0)$ \\
\hline Q5 & $2.13(.74)$ & $2.24(.86)$ & $2.29(.75)$ & $2.38(.75)$ & $2.20(.81)$ & $2.39(.89)$ & $2.37(.83)$ & $2.36(.90)$ \\
\hline$r$ & $-.26 * *$ & $-.25 * *$ & $-.30 * *$ & $-.33 * *$ & $-.19 * *$ & $-.23 * *$ & $-.24 * *$ & $-.25 * *$ \\
\hline \multicolumn{9}{|c|}{ Elterliches Unterstützungs- und Erziehungsverhalten } \\
\hline gering & $2.71(.77)$ & $3.06(.80)$ & $3.27(1.0)$ & $3.15(.68)$ & $2.88(.99)$ & $3.00(.94)$ & $3.07(1.1)$ & $3.50(.95)$ \\
\hline mittel & $2.50(.85)$ & $2.80(.91)$ & $2.94(.84)$ & $2.89(.85)$ & $2.48(.96)$ & $2.91(.96)$ & $2.90(.96)$ & $2.90(.98)$ \\
\hline hoch & $2.30(.88)$ & $2.42(.82)$ & $2.57(.81)$ & $2.63(.86)$ & $2.38(.94)$ & $2.54(.92)$ & $2.70(.91)$ & $2.70(1.0)$ \\
\hline$r$ & $-.14 * *$ & $-.21 * *$ & $-.22 * *$ & $-.17 * *$ & $-.07^{\hat{\imath}}$ & $-.18 * *$ & $-.10 * *$ & $-.16 * *$ \\
\hline
\end{tabular}

Anmerkung. $\mathrm{MH}=$ Migrationshintergrund. $* p<.05, * * p<.01, \dddot{\imath} p<.10$. Korrelationen nach Pearson (Migrationshintergrund) bzw. Spearman. 
Tabelle 50. Zusammenhänge Schulnoten in Sachkunde/Englisch und Sport mit sozial-strukturellen Merkmalen.

\begin{tabular}{|c|c|c|c|c|c|c|c|c|}
\hline & \multicolumn{2}{|c|}{ SK } & \multicolumn{2}{|c|}{$\mathbf{E}$} & \multicolumn{4}{|c|}{ Sport } \\
\hline & 3. & 4. & 5. & 6. & 3. & 4. & 5. & 6. \\
\hline & $M(S D)$ & $M(S D)$ & $M(S D)$ & $M(S D)$ & $M(S D)$ & $M(S D)$ & $M(S D)$ & $M(S D)$ \\
\hline \multicolumn{9}{|c|}{ Migrationshintergrund } \\
\hline Deutsch & $2.16(.80)$ & $2.25(.80)$ & $2.46(.96)$ & $2.62(.96)$ & $1.94(.74)$ & $2.12(.77)$ & $2.10(.79)$ & $2.04(.78)$ \\
\hline MH & $2.66(.95)$ & $2.81(.99)$ & $2.81(1.0)$ & $2.89(1.1)$ & $2.30(.81)$ & $2.40(.73)$ & $2.22(.77)$ & $2.15(.81)$ \\
\hline$r$ & $.25 * *$ & $.27 * *$ & $.16^{* *}$ & $.12 * *$ & $.20 * *$ & $.16 * *$ & $.07^{i}$ & $.06^{\mathrm{ns}}$ \\
\hline \multicolumn{9}{|c|}{ Bildungsniveau } \\
\hline niedrig & $2.67(.88)$ & $2.84(.76)$ & $3.12(.90)$ & $3.14(1.0)$ & $2.38(.93)$ & $2.49(.80)$ & $2.28(.77)$ & $2.22(.86)$ \\
\hline mittel & $2.36(.81)$ & $2.47(.86)$ & $2.62(.92)$ & $2.86(.95)$ & $2.08(.78)$ & $2.22(.77)$ & $2.18(.81)$ & $2.16(.81)$ \\
\hline hoch & $2.06(.78)$ & $2.18(.84)$ & $2.25(.92)$ & $2.38(.94)$ & $1.88(.67)$ & $2.04(.72)$ & $2.00(.73)$ & $1.93(.74)$ \\
\hline$r$ & $-.24 * *$ & $-.24 * *$ & $-.29 * *$ & $-.29 * *$ & $-.19 * *$ & $-.19 * *$ & $-.14 * *$ & $-.15^{* *}$ \\
\hline \multicolumn{9}{|c|}{ Wohlstand } \\
\hline Q1 & $2.61(.86)$ & $2.82(.90)$ & $2.88(.98)$ & $3.01(1.1)$ & $2.25(.79)$ & $2.32(.76)$ & $2.23(.78)$ & $2.20(.79)$ \\
\hline Q2 & $2.48(.90)$ & $2.60(.92)$ & $2.71(.95)$ & $2.96(1.0)$ & $2.15(.89)$ & $2.27(.74)$ & $2.23(.76)$ & $2.12(.81)$ \\
\hline Q3 & $2.26(.80)$ & $2.40(.76)$ & $2.61(.92)$ & $2.71(.94)$ & $2.04(.75)$ & $2.26(.80)$ & $2.24(.75)$ & $2.18(.78)$ \\
\hline Q4 & $2.06(.81)$ & $2.09(.75)$ & $2.40(1.0)$ & $2.50(.94)$ & $1.90(.71)$ & $2.04(.73)$ & $1.98(.81)$ & $1.95(.79)$ \\
\hline Q5 & $2.02(.78)$ & $2.08(.87)$ & $2.13(.88)$ & $2.31(.89)$ & $1.84(.63)$ & $2.01(.72)$ & $1.96(.76)$ & $1.88(.74)$ \\
\hline$r$ & $-.26 * *$ & $-.32 * *$ & $-.25 * *$ & $-.26^{* *}$ & $-.19 * *$ & $-.16^{* *}$ & $-.15^{* *}$ & $-.15^{* *}$ \\
\hline \multicolumn{9}{|c|}{$\begin{array}{l}\text { Elterliches Unterstützungs- und } \\
\text { Erziehungsverhalten }\end{array}$} \\
\hline gering & $2.59(.62)$ & $2.88(.93)$ & $3.33(1.0)$ & $3.23(.66)$ & $2.18(.88)$ & $2.50(.76)$ & $2.53(.64)$ & $2.65(.85)$ \\
\hline mittel & $2.34(.83)$ & $2.69(.89)$ & $2.69(1.0)$ & $2.88(1.0)$ & $2.15(.78)$ & $2.39(.82)$ & $2.21(.83)$ & $2.11(.79)$ \\
\hline hoch & $2.22(.88)$ & $2.26(.85)$ & $2.46(.94)$ & $2.51(.97)$ & $1.95(.75)$ & $2.12(.74)$ & $2.08(.76)$ & $2.01(.77)$ \\
\hline$r$ & $-.10 * *$ & $-.24 * *$ & $-.14 * *$ & $-.21 * *$ & $-.12^{* *}$ & $-.16^{* *}$ & $-.09 *$ & $-.11 *$ \\
\hline
\end{tabular}

Anmerkung. $\mathrm{MH}=$ Migrationshintergrund. ${ }^{*} p<.05, * * p<.01, \dddot{\imath} p<.10$, ns $=$ nicht signifikant. Korrelationen nach Pearson (Migrationshintergrund) bzw. Spearman.

Da sich nicht nur die unterschiedlichen sozial-strukturellen Variablen gegenseitig bedingen, sondern auch die verschiedenen Mediennutzungsvariablen (auch zum Teil stärker) mit diesen korrelieren, soll die gegenseitige Abhängigkeit dieser sowie deren Zusammenhang mit Schulnoten unter der Berücksichtigung wei- 
terer Schülervariablen in einem Strukturgleichungsmodell ${ }^{100}$ anhand der Daten der Befragungswelle der fünften Klasse dargestellt werden (Abbildung 51). Die Daten der fünften Klasse wurden gewählt, da zu diesem Messzeitpunkt zum letzten Mal eine Intelligenztestung durchgeführt wurde. Bei guter Passungsgüte des Modells $\left(\chi^{2}=128.69(62, N=722), R M S E A=.04, R M R=.04, S R M R=.04\right.$, $G F I=.98, A G F I=.96)$ und einer Varianzaufklärung von 33 Prozent haben die kognitiven Fähigkeiten (CFT 20) die größte Erklärungskraft für die mittleren Schulnoten in Deutsch, Mathematik und Englisch $(\beta=.38)$. Die korrelativen Auswertungen legten eine ebenfalls vorrangige Bedeutung des Bildungshintergrundes im Elternhaus, welcher maßgeblich mit den kognitiven Fähigkeiten der Kinder zusammenhängt $(\beta=.19)$, nahe, was in diesem multifaktoriellen Modell bestätigt werden konnte $(\beta=.18)$.

Als weiterer Einflussfaktor auf die mittleren Schulnoten in Deutsch, Mathematik und Englisch zeigte sich auf Seiten der Kinder ein hohes Selbstkonzept eigener Schulfähigkeiten $(\varphi=.46)$ sowie eine positive Einstellung zur Schule $(\varphi=$. 18 ), wobei letztere auch mit einem geringeren Konsum gewalthaltiger Medieninhalte $(\varphi=-.12)$ sowie mit geringeren Mediennutzungszeiten $(\beta=-.21)$ in dem Sinne, dass Kinder mit geringeren Mediennutzungszeiten eine bessere Einstellung zur Schule haben, zusammenhängt. Das Geschlecht der Grundschulkinder hat keine eigenständige Erklärungskraft für bestehende Schulleistungsunterschiede $\left(\beta=-.05^{\text {ns }}\right)$.

Bei gleichzeitiger Betrachtung der im Modell enthaltenen Variablen verliert auf Seiten des sozialen und kulturellen Kapitals sowohl der Wohlstand in der Fa-

100 Eine vorgeschaltete Analyse der Mehrebenenstruktur der Daten (Level 1: Messzeitpunkte, Level 2: Individuen, Level 3: Klassen) in STATA (Version SE 10) erbrachte für das Null-Modell, d. h. ein Modell ohne erklärende Variablen, eine Intraklassenkorrelation (ICC) von.11 auf Level 3, d. h. maximal $11 \%$ der Varianz der mittleren Noten kann durch Merkmale des Klassenkontextes erklärt werden (vgl. Hox, 2002; Snijders \& Bosker, 1999). Nach Oberwittler (2003) sollten bei Betrachtung der Intraklassenkorrelationen die wichtigsten sozio-demografischen Parameter in Ergänzung zum Null-Modell auf Individualebene eingeführt werden, da nur solche Intraklassenkorrelationen inhaltlich bedeutsam sind, die auch nach Einführung wesentlicher Merkmale der Soziodemografie auf Individualebene bestehen bleiben. Diese sog. konditionale Intraklassenkorrelation (Oberwittler, 2003) reduziert sich im vorliegenden Datensatz nach Einführung der Variablen des sozialen und kulturellen Kapitals (Bildungsniveau im Elternhaus, Wohlstand im Elternhaus, Migrationshintergrund) um 7.7\% Prozentpunkte auf 3.3\%. Angesichts dieses deutlichen Rückgangs und der geringen maximalen Varianz, die auf Level 3 aufgeklärt werden kann, wurde auf eine Berücksichtigung der Klassenebene in den berechneten Strukturgleichungsmodellen verzichtet. Darüber hinaus ist es nicht Anliegen der vorliegenden Untersuchung, mögliche Einflussfaktoren auf Schulleistungen inklusive Effekten des Klassenkontextes in ihrer Gänze aufzuklären (dies wurde bereits an anderer Stelle geleistet, vgl. Helmke \& Weinert, 1997; Weinert \& Helmke, 1997; Schrader \& Helmke, 2008), sondern es geht vielmehr darum, mögliche Effekte der häuslichen Mediennutzung unter Berücksichtigung der wichtigsten Einflussvariablen der Individualebene zu bestimmen. 
milie $\left(\beta=.03^{\mathrm{ns}}\right)$, der stark mit dem Bildungsniveau verbunden ist $(\varphi=.39)$, als auch der Migrationshintergrund der Kinder an eigenständiger Erklärungskraft. Der Migrationshintergrund im Elternhaus wirkt sich wiederum nur indirekt dadurch aus, dass dieser zum einen mit geringerem Wohlstand $(\beta=-.37)$ und zum anderen etwas weniger ausgeprägt mit einem niedrigeren Bildungsniveau $(\beta=-$. 09) zusammenhängt. Gemäß diesem Modell haben Kinder mit Migrationshintergrund aber nicht nur aufgrund ihres Migrationshintergrundes schlechtere Noten $\left(\beta=-.05^{\text {ns }}\right)$. Die Zusammenhänge zwischen den Variablen des sozialen und kulturellen Kapitals und Mediennutzungsvariablen wurden bereits unter Kapitel 2.2.5 (vgl. Abbildung 47) beschrieben und bewegen sich in diesem Modell auf vergleichbarem Niveau.

In der gewählten Modellstruktur findet sich zudem bei gleichzeitiger Kontrolle der Variablen des sozialen und kulturellen Kapitals sowie der wichtigsten Variablen auf Seiten des Schülers ein signifikanter schwacher bis mittlerer Einfluss des Spielens gewalthaltiger Computerspiele bzw. des Betrachtens gewalthaltiger Filme $(\beta=-.17)$ auf die Schulleistungen der befragten Kinder, aber kein signifikanter direkter Einfluss der reinen Mediennutzungszeiten $\left(\beta=-.02^{\text {ns }}\right)$. Diese bedingen jedoch eine deutlich häufigere Nutzung von Gewaltmedien $(\beta=.33)$ und entfalten somit über diesen Pfad wie auch über eine negativere Schuleinstellung ihre Wirkung. Aus den bivariaten Auswertungen wie auch aus dem Modell ergibt sich ferner, dass es vornehmlich Jungen sind, die ein gewaltbetontes Nutzungsprofil mit hohen Nutzungszeiten aufweisen (Nutzungszeiten: $\beta=.26$; Gewaltmediennutzung: $\beta=.43$; siehe auch Abbildung 47). Letztlich können auch über das elterliche Erziehungs- und Unterstützungsverhalten Schulleistungen zum Teil erklärt werden; der Zusammenhang liegt dabei in einer etwas geringeren Größenordnung als bei der Gewaltmediennutzung $(\beta=.12)$ : Berichten Kinder über ein hohe elterliche Zuwendung, geht dies nicht nur mit besseren Schulnoten, sondern auch mit einer geringeren Nutzung von Gewaltmedien $(\beta=-.12)$ und geringeren Nutzungszeiten $(\beta=-.12)$ einher. 


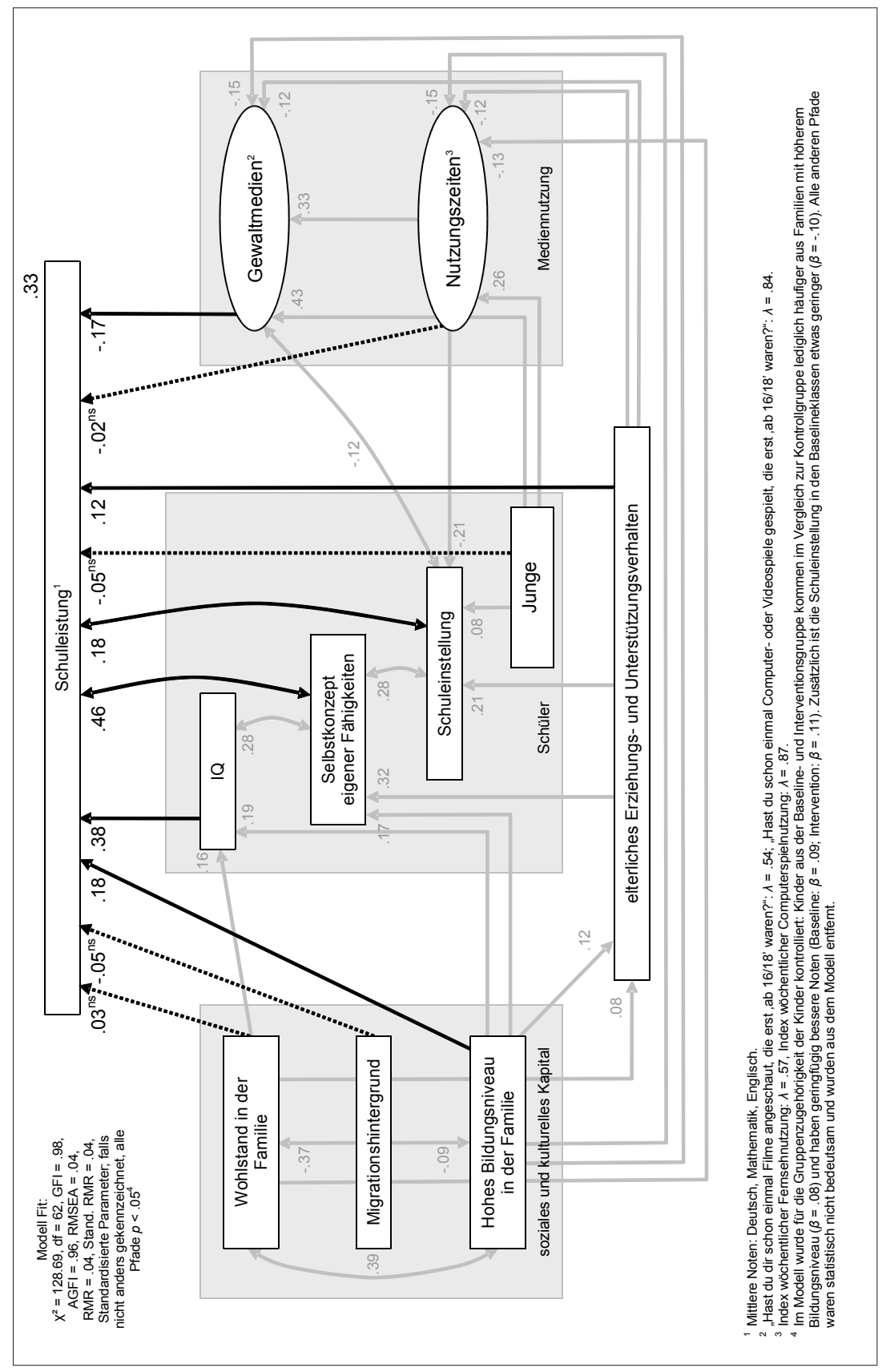

Abbildung 51. Bedingungsfaktoren schulischer Leistungen (5. Klasse). 


\subsubsection{Mediennutzung und Schulleistung - querschnittlicher Zusammenhang}

Bei der Betrachtung des Zusammenspiels der unterschiedlichen Medienvariablen - Ausstattung, Nutzungszeiten, Inhalte und Erziehung - mit den bedeutsamsten sozial-strukturellen Merkmalen (vgl. Abbildung 47) hat sich neben dem Bildungshintergrund im Elternhaus das Geschlecht der Kinder als wichtigster Einflussfaktor erwiesen. So sind es vor allem Jungen, die mit eigenen Geräten im Kinderzimmer ausgestattet sind, höhere Nutzungszeiten aufweisen und insbesondere deutlich häufiger gewalthaltige Medien konsumieren (vgl. Kapitel 2.2). Ein Defizit obigen Rahmenmodells schulischer Leistungen (vgl. Abbildung 51) besteht nun möglicherweise darin, dass durch eine über Fernsehen und Computerspiele gemittelte Betrachtung der Mediennutzungsgewohnheiten, ${ }^{101}$ Geschlechtereffekte und somit nach Medien differierende Nutzungsmuster - trotz einer Berücksichtigung des Geschlechts der Kinder im Modell - nicht ausreichend abgebildet wurden und Informationen dadurch verloren gingen.

Um die gefundenen Zusammenhänge zwischen sozial-strukturellen Variablen, Mediennutzungsgewohnheiten und Schulnoten sowie insbesondere zwischen Mediennutzungsgewohnheiten und Schulnoten differenzierter betrachten zu können, wurden deshalb wiederum mit den Daten der fünften Klasse ${ }^{102}$ zwei getrennte Strukturgleichungsmodelle für Jungen und Mädchen berechnet, welche neben den Medienvariablen, die nach Medium sowie Inhalt und Nutzungszeiten getrennt wurden, jene Variablen beinhalten, die sich in den bi- und multivariaten Analysen als am bedeutsamsten erwiesen haben (vgl. Abbildung 52), d. h. das Bildungsniveau im Elternhaus sowie aufseiten der Kinder die kognitiven Fähigkeiten, gemessen mit dem CFT 20.

Bei guter Passungsgüte (Jungen: $\chi 2=6.01$ (9, $N=363$ ), $R M S E A=.00$, $R M R=.01, S R M R=.03, G F I=.99, A G F I=.98$; Mädchen: $\chi 2=3.70(8, N=359)$, $R M S E A=.00, R M R=.02, S R M R=.02, G F I=.99, A G F I=.99)$ und einer Varianzaufklärung von 32 (Jungen) bzw. 35 Prozent (Mädchen) haben erwartungsgemäß für beide Geschlechter wiederum die kognitiven Fähigkeiten (CFT 20) die größte Erklärungskraft für die mittleren Schulnoten in Deutsch, Mathematik und Englisch (siehe auch Abbildung 51), wobei diesem Faktor bei den Mädchen ( $\beta=$ 42) noch eine größere Bedeutung zukommt als bei den Jungen $(\beta=.35)$. Wie bereits aufgrund der obigen Auswertungen zu erwarten war, ist auch der Bildungshintergrund im Elternhaus, welcher wiederum maßgeblich mit den kognitiven Fähigkeiten der Kinder zusammenhängt (Jungen: $\beta=.20$; Mädchen: $\beta=.29$ ), sowohl bei der Erklärung von Unterschieden in Mediennutzungsgewohnheiten als

101 Aufgrund der Vielzahl weiterer Variablen, wurde in diesem Modell zur Komplexitätsreduktion mit Summenvariablen beider Medien gearbeitet.

102 In der fünften Klasse wurde zum letzten Mal eine Intelligenztestung durchgeführt. 
auch bei der Erklärung von Schulleistungsunterschieden für beide Geschlechter gleichermaßen von Relevanz (siehe auch Abbildung 47 und Abbildung 51). In beiden Strukturgleichungsmodellen beachtenswert ist jedoch die Tatsache, dass bei einer nach Geschlecht und Medien getrennten Betrachtung der Mediennutzungsgewohnheiten der Kinder, bei Berücksichtigung der beiden stärksten Einflussfaktoren auf Schulnoten (kognitive Fähigkeiten der Kinder sowie Bildungshintergrund im Elternhaus) sowohl den Medieninhalten als auch den Mediennutzungszeiten eine substanzielle eigenständige, wenn auch geringere, Erklärungskraft für die mittleren Schulnoten zukommt. Interessanterweise tragen die beiden Medien Fernsehen und Computerspiele je nach Geschlecht unterschiedlich zur Erklärung bei: Bei den Jungen wirken sich vor allem die Fernsehnutzungszeiten $(\beta=-.12)$ sowie Gewaltinhalte in Computerspielen $(\beta=-.17)$ negativ auf deren Schulnoten aus. Bei den Mädchen sind es hingegen Gewaltinhalte im Fernsehen ( $\beta=-.14$ ) sowie die Computerspielnutzungszeiten $\left(\beta=-.08^{n s}\right)$, die eine Wirkung auf die mittleren Noten zeigen. 


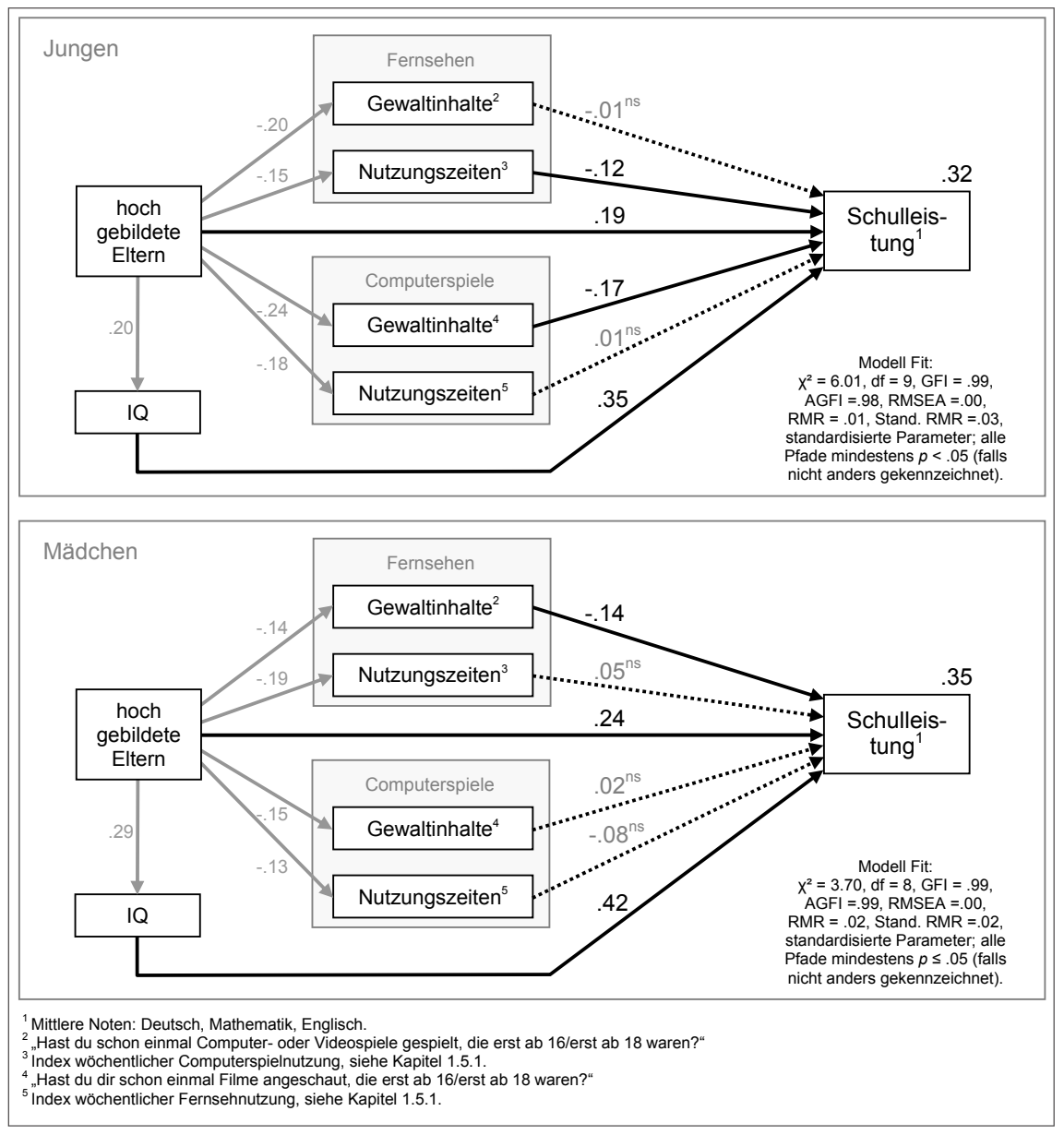

\section{Abbildung 52. Strukturgleichungsmodelle zur Erklärung des Einflusses von Mediennutzung auf Schulnoten getrennt nach Geschlecht (5. Klasse). ${ }^{103}$}

103 In beiden Modellen wurde für die Gruppenzugehörigkeit der Kinder kontrolliert. Jungen: Jungen aus der Baselinegruppe kommen im Vergleich zur Kontrollgruppe lediglich häufiger aus Familien mit höherem Bildungsniveau $(\beta=.13)$ und haben geringfügig bessere Noten $(\beta=.15)$; Alle anderen Pfade waren statistisch nicht bedeutsam und wurden aus dem Modell entfernt. Mädchen: Mädchen aus der Interventionsgruppe haben im Vergleich zur Kontrollgruppe geringfügig bessere Noten $(\beta=.13)$, geringere Computerspielzeiten $(\beta=-.12)$ und einen geringeren Gewaltcomputerspielkonsum $(\beta=-$. 13); Alle anderen Pfade waren statistisch nicht bedeutsam und wurden aus dem Modell entfernt. 
Dieser Unterschied kann u. a. dadurch erklärt werden, dass bei gleichzeitiger Betrachtung von Zeiten und Inhalten das Merkmal mit der für das jeweilige Medium größeren Heterogenität in den Vordergrund tritt. Für Jungen gehört der Gewaltmedienkonsum im Fernsehen zur Realität (69\% der Jungen bejahten diese Frage), in den Nutzungszeiten sind jedoch größere Unterschiede zwischen den Jungen zu beobachten. Auch Gewalt in Computerspielen (66\% der Jungen bejahten diese Frage) gehört zur Normalität der Jungen, ist aber dennoch im Vergleich zu den Nutzungszeiten das unterscheidendere Merkmal. Hinsichtlich der Nutzungszeiten sind zudem deutlich mehr Jungen in den Gruppen mit exzessiven Fernsehzeiten als mit exzessiven Computerspielnutzungszeiten zu finden, weswegen sich die Fernsehzeiten stärker auswirken. Bei annähernd gleichen Häufigkeiten der Gewaltmediennutzung (69\% bzw. 66\%) scheint sich ein Gewaltmedienkonsum im Bereich der Computerspiele somit für die Jungen zum Teil zum Fernsehen zu unterscheiden. Dies könnte entweder darin begründet liegen, dass sich die Gruppe der Jungen, die gewalthaltige Computerspiele spielt, von der Gruppe mit gewalthaltigem Fernsehkonsum unterscheidet (bei einer mittleren Korrelation beider Medien von $r=.46$ ) oder dass sich der Gewaltmedienkonsum in Computerspielen inhaltlich oder in seiner Wirkung von der alltäglich konsumierten Fernsehgewalt unterscheidet. Für Mädchen ist hingegen der „Nicht-Konsum“ von Gewalt in Computerspielen die Normalität (nur 19\% der Mädchen bejahten diese Frage), wohingegen sich die Nutzungszeiten dieses Mediums deutlicher unterscheiden. Bezogen auf das Fernsehen sind die Nutzungszeiten annähernd normal verteilt und im Vergleich zum Computerspielen deutlich homogener, weshalb sich der Gewaltmedienkonsum im Fernsehen (50\% der Mädchen bejahten diese Frage) stärker auf die mittleren Noten auswirken kann. Bei einer gemittelten Betrachtung der Mediennutzungszeiten führte dies dazu, dass diese keine Erklärungskraft für Schulleistungsunterschiede mehr besitzen.

Für die beiden Kernfächer Deutsch und Mathematik sollen die Zusammenhänge zwischen spezifischen Mediennutzungsgewohnheiten und Schulnoten, hier am Beispiel der Fernsehzeiten, noch einmal getrennt für beide Geschlechter grafisch veranschaulicht werden (siehe Abbildung 53). 


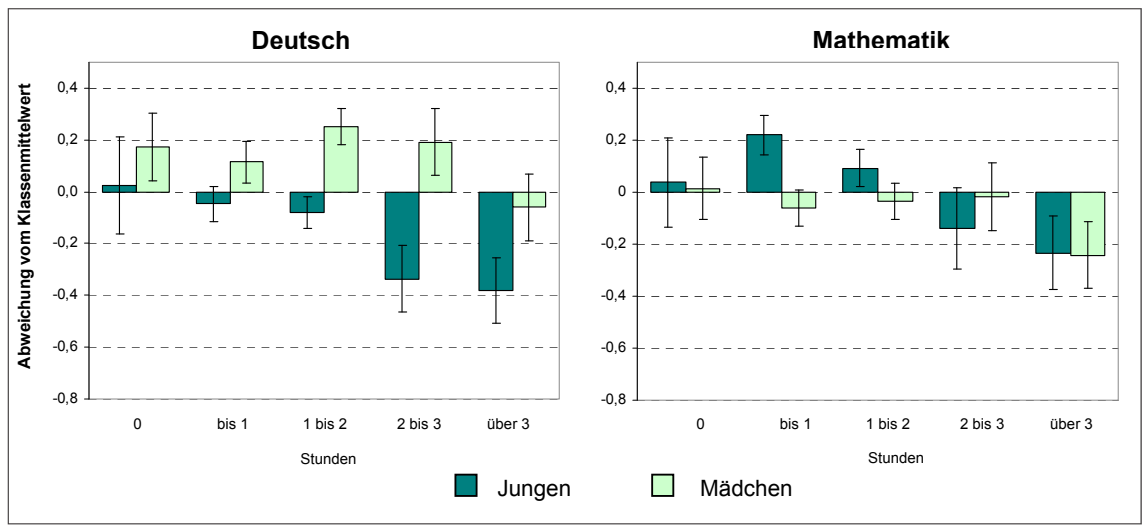

Abbildung 53. Notenabweichungen von Klassenmittewert in Deutsch und Mathematik nach Geschlecht und Fernsehnutzungszeiten an einem gewöhnlichen Schultag (5. Klasse). Fehlerbalken zeigen Standardfehler des Mittelwerts.

Wie Abbildung $53 \mathrm{zu}$ entnehmen ist, stellt sich der Zusammenhang zwischen den Fernsehnutzungszeiten und Schulnoten bei Betrachtung der beiden Kernfächer für die Geschlechter unterschiedlich dar (vgl. Abbildung 50 sowie Tabelle 49 und Tabelle 50 für die mittleren Noten). Vor allem für die Jungen bedeuten erhöhte Fernsehzeiten sowohl in Deutsch als auch in Mathematik geringere Schulleistungen. Der größte Leistungseinbruch ist dabei in beiden Fächern bei täglichen Fernsehzeiten von über 2 Stunden an einem Schultag zu beobachten. So werden die deutlich schlechtesten Leistungen im Vergleich zum Klassenmittelwert sowohl in Mathematik als auch in Deutsch von Jungen mit einem Fernsehkonsum von über zwei bzw. drei Stunden an einem Schultag erbracht. In Mathematik ist die Verteilung durch insgesamt bessere Leistungen der Jungen in diesem Fach, lediglich etwas nach oben verschoben. Die Leistungen der Mädchen sind in beiden Fächern im Vergleich zu den Jungen über die zeitlichen Nutzergruppen homogener, in Mathematik lediglich insgesamt auf einem im Vergleich zum Klassenmittelwert geringeren Niveau, hier kommen die jeweils geschlechtstypischen „Achillesfersenfächer“ zum Ausdruck. Deutliche Leistungseinbußen im mittleren Nutzungsbereich wie für die Jungen sind für die Mädchen jedoch nicht festzustellen. So waren auch in den obigen Strukturgleichungsmodellen (vgl. Abbildung 52) die Fernsehnutzungszeiten insbesondere bei den Jungen von Relevanz, bei den Mädchen hingegen die Computerspielnutzungszeiten. Aber auch für die Mädchen gilt: Die deutlich schlechtesten Leistungen im Vergleich zum Klassenmittelwert werden sowohl in Mathematik als auch 
in Deutsch von Mädchen mit einem täglichen Fernsehkonsum von über drei Stunden an einem Schultag erbracht.

\subsubsection{Mediennutzung und Schulleistung - längsschnittlicher Zusammenhang}

Die Querschnittsbefunde aus Kapitel 3.2.2 und 3.2.3 zeigen somit, unter Berücksichtigung weiterer aus der Literatur bekannter Einflussvariablen, deutliche $\mathrm{Zu}$ sammenhänge zwischen einer inhaltlich problematischen (wenn nach Geschlecht getrennt wird) sowie zeitlich exzessiven Mediennutzung und Schulnoten im Kindesalter. Ziel der weiteren Ausführungen soll es sein, anhand der Längsschnittdaten empirisch begründete Aussagen über die genaue Richtung dieser Zusammenhänge zu machen.

Um einen ersten Eindruck zur differenziellen Bedeutung der einzelnen Mediennutzungsvariablen im Zusammenspiel mit den erbrachten Schulleistungen zu bekommen, wurden zunächst quer- sowie längsschnittliche Partialkorrelationen zwischen den Schulnoten in den Fächern Deutsch, Mathematik, Sachkunde und Englisch sowie den unterschiedlichen Medienvariablen ${ }^{104}$ berechnet. Kontrolliert wurde dabei für den Einfluss der kognitiven Fähigkeiten der Kinder am Ende der dritten Klasse (CFT 20) sowie für den familiären Wohlstand (siehe Tabelle 51). Der familiäre Wohlstand wurde dem Bildungsniveau im Elternhaus als weitere Einflussvariable vorgezogen, da dieser einen geringeren Überschneidungsbereich mit den kognitiven Fähigkeiten der Kinder aufweist, aber dennoch in vergleichbarem Maß mit den Schulnoten zusammenhängt (wenn das Bildungsniveau nicht zusätzlich betrachtet wird, vgl. Tabelle 49 und Tabelle 50).

Bezüglich der durchschnittlichen wöchentlichen Mediennutzungszeiten an einem Schultag zeigen sich zu allen Messzeitpunkten, bei Kontrolle der kognitiven Fähigkeiten sowie des familiären Wohlstands, vor allem für das Computerspielen negative Zusammenhänge mit den Noten in Deutsch, Sachkunde und Englisch und zu einem geringeren Ausmaß mit den Noten in Mathematik. In der längsschnittlichen Betrachtung deuten die Korrelationen zwischen den Computerspielnutzungszeiten und den Noten tendenziell eher auf eine verstärkende Wirkung der Computerspielnutzungszeiten auf schlechtere Noten hin, als auf eine Selektion in dem Sinne, dass Kinder mit schlechten Schulnoten verstärkt exzessiv Computerspiele spielen. Den stärksten Einfluss entwickeln dabei die

104 Fernsehzeit und Computerspielzeit wurden über den Index wöchentliche Fernsehnutzung und den Index wöchentliche Computerspielnutzung erhoben. Gewaltmedienkonsum im Fernsehen wurde über die Frage „Hast du schon einmal Filme angeschaut die erst ab 16/18 waren?“, Gewaltmedienkonsum in Computerspielen wurde über die Frage „Hast du schon einmal Computerspiele gespielt die erst ab 16/18 waren?" operationalisiert. 
Computerspielnutzungszeiten der dritten Klasse, d. h. sehr frühe exzessive Nutzungszeiten.

Für die Fernsehnutzungszeiten können hingegen über die Messzeitpunkte hinweg kaum statistisch bedeutsame Korrelationen beobachtet werden. Lediglich die Zeiten der dritten, vierten und fünften Klasse scheinen von Bedeutung zu sein. Schlussfolgerungen hinsichtlich der Wirkrichtung wie für die Computerspielzeiten können jedoch für die Fernsehnutzungszeiten nicht gezogen werden. Bezüglich der konsumierten Gewaltinhalte ergibt sich indes ein eindeutigeres Bild. Bei Kontrolle der kognitiven Fähigkeiten sowie des familiären Wohlstands können sowohl für das Computerspielen als auch für den Fernsehkonsum negative Zusammenhänge mit den Noten in Deutsch, Sachkunde und Englisch sowie für das Fernsehen mit den Noten in Mathematik festgestellt werden. In der längsschnittlichen Betrachtung legen diese Korrelationen zwischen den beiden Medien und den Noten eine Wechselwirkung nahe, d. h. Kinder mit schlechten Schulnoten spielen verstärkt gewalthaltige Computerspiele und schauen verstärkt gewalthaltige Fernsehprogramme, was wiederum zu schlechteren Noten führt.

Tabelle 51. Partialkorrelationen Mediennutzung (Computerspiele, Fernsehen) und Schulnoten.

\begin{tabular}{|c|c|c|c|c|c|c|c|c|}
\hline \multirow{2}{*}{ Klasse } & \multicolumn{4}{|c|}{ Nutzungszeiten } & \multicolumn{4}{|c|}{ Inhalte } \\
\hline & 3. & 4. & 5. & 6. & 3. & 4. & 5. & 6. \\
\hline \multicolumn{9}{|c|}{ Computerspiele } \\
\hline \multicolumn{9}{|c|}{ Deutsch } \\
\hline 3. Klasse & $-.17 * *$ & $-.14 * *$ & $-.17 * *$ & $-.10^{*}$ & $-.16 * *$ & $-.13 * *$ & $-.09 * *$ & $-.18 * *$ \\
\hline 4. Klasse & $-.18 * *$ & $-.12^{* *}$ & $-.09^{* *}$ & $-.09 *$ & $-.12 *$ & $-.16 * *$ & $-.16^{*}$ & $-.21 * *$ \\
\hline 5. Klasse & $-.27 * *$ & $-.16^{* *}$ & $-.20 * *$ & $-.17 * *$ & $-.19 * *$ & $-.21 * *$ & $-.23 * *$ & $-.23 * *$ \\
\hline 6. Klasse & $-.27 * *$ & $-.14 * *$ & $-.18 * *$ & $-.15 * *$ & $-.16 * *$ & $-.17 * *$ & $-.21 * *$ & $-.18 * *$ \\
\hline \multicolumn{9}{|c|}{ Mathematik } \\
\hline 3. Klasse & $-.05^{\mathrm{ns}}$ & $.03^{\mathrm{ns}}$ & $-.04^{\mathrm{ns}}$ & $.07^{\mathrm{ns}}$ & $-.02^{\mathrm{ns}}$ & $.04^{\mathrm{ns}}$ & $.10^{*}$ & $.03^{\text {ns }}$ \\
\hline 4. Klasse & $-.05^{\mathrm{ns}}$ & $-.02^{\mathrm{ns}}$ & $-.03^{\mathrm{ns}}$ & $.05^{\mathrm{ns}}$ & $-.08^{\hat{\imath}}$ & $-.03^{\mathrm{ns}}$ & $.01^{\mathrm{ns}}$ & $-.05^{\mathrm{ns}}$ \\
\hline 5. Klasse & $-.09 *$ & $-.02^{\mathrm{ns}}$ & $-.09^{i}$ & $-.05^{\mathrm{ns}}$ & $-.05^{\mathrm{ns}}$ & $-.03^{\mathrm{ns}}$ & $-.11 *$ & $-.10 *$ \\
\hline 6. Klasse & $-.10 *$ & $-.06^{\mathrm{ns}}$ & $-.10 * *$ & $-.05^{\mathrm{ns}}$ & $-.09 *$ & $-.01^{\mathrm{ns}}$ & $-.08^{i}$ & $-.11 *$ \\
\hline \multicolumn{9}{|c|}{ Sachkunde } \\
\hline 3. Klasse & $-.19 * *$ & $-.11 * *$ & $-.13 * *$ & $-.03^{\mathrm{ns}}$ & $-.15 * *$ & $-.08^{\hat{\imath}}$ & $-.10 * *$ & $-.15^{* *}$ \\
\hline 4. Klasse & $-.20 * *$ & $-.14 * *$ & $-.08^{i}$ & $-.03^{\mathrm{ns}}$ & $-.13 * *$ & $-.16 * *$ & $-.08^{\Uparrow i}$ & $-.13 * *$ \\
\hline \multicolumn{9}{|l|}{ Englisch } \\
\hline 5. Klasse & $-.18 * *$ & $-.11 * *$ & $-.21 * *$ & $-.15 * *$ & $-.10 * *$ & $-.14 * *$ & $-.21 * *$ & $-.18 * *$ \\
\hline 6. Klasse & $-.23 * *$ & $-.14 * *$ & $-.21 * *$ & $-.16^{* *}$ & $-.20 * *$ & $-.15^{* *}$ & $-.20 * *$ & $-.20 * *$ \\
\hline
\end{tabular}




\begin{tabular}{|c|c|c|c|c|c|c|c|c|}
\hline \multirow[b]{2}{*}{ Klasse } & \multicolumn{4}{|c|}{ Nutzungszeiten } & \multicolumn{4}{|c|}{ Inhalte } \\
\hline & 3. & 4. & 5. & 6. & 3. & 4. & 5. & 6. \\
\hline \multicolumn{9}{|c|}{ Fernsehen } \\
\hline \multicolumn{9}{|l|}{ Deutsch } \\
\hline 3. Klasse & $-.06^{\mathrm{ns}}$ & $-.09 *$ & $-.10 *$ & $-.02^{\mathrm{ns}}$ & $-.18 * *$ & $-.06^{\mathrm{ns}}$ & $-.09 *$ & $-.14 * *$ \\
\hline 4. Klasse & $-.04^{\mathrm{ns}}$ & $-.13 * *$ & $-.07^{i}$ & $-.05^{\mathrm{ns}}$ & $-.20 * *$ & $-.13 * *$ & $-.10 * *$ & $-.16^{* *}$ \\
\hline 5. Klasse & $-.12 * *$ & $-.13 * *$ & $-.12 * *$ & $-.06^{\mathrm{ns}}$ & $-.19 * *$ & $-.17 * *$ & $-.18^{* *}$ & $-.15 * *$ \\
\hline 6. Klasse & $-.09 *$ & $-.05^{\mathrm{ns}}$ & $-.12 * *$ & $-.05^{\mathrm{ns}}$ & $-.22 * *$ & $-.05^{\mathrm{ns}}$ & $-.18 * *$ & $-.14 * *$ \\
\hline \multicolumn{9}{|c|}{ Mathematik } \\
\hline 3. Klasse & $.00^{\mathrm{ns}}$ & $-.06^{\mathrm{ns}}$ & $-.04^{\mathrm{ns}}$ & $.02^{\text {ns }}$ & $-.08 *$ & $.01^{\mathrm{ns}}$ & $-.03^{\mathrm{ns}}$ & $-.02^{\mathrm{ns}}$ \\
\hline 4. Klasse & $.03^{\mathrm{ns}}$ & $-.04^{\mathrm{ns}}$ & $-.04^{\mathrm{ns}}$ & $.06^{\mathrm{ns}}$ & $-.16 * *$ & $-.06^{\mathrm{ns}}$ & $-.04^{\mathrm{ns}}$ & $-.06^{\mathrm{ns}}$ \\
\hline 5. Klasse & $-.02^{\mathrm{ns}}$ & $-.02^{\mathrm{ns}}$ & $-.11 * *$ & $-.04^{\mathrm{ns}}$ & $-.16 * *$ & $-.11 * *$ & $-.14 * *$ & $-.12 * *$ \\
\hline 6. Klasse & $-.02^{\mathrm{ns}}$ & $-.04^{\mathrm{ns}}$ & $-.14 * *$ & $-.06^{\mathrm{ns}}$ & $-.16^{* *}$ & $-.08^{\grave{\imath}}$ & $-.10 * *$ & $-.12 * *$ \\
\hline \multicolumn{9}{|c|}{ Sachkunde } \\
\hline 3. Klasse & $-.07^{i}$ & $-.08 *$ & $-.07^{\mathrm{ns}}$ & $.00^{\mathrm{ns}}$ & $-.14 * *$ & $-.05^{\mathrm{ns}}$ & $-.08^{i}$ & $-.14 * *$ \\
\hline 4. Klasse & $-.03^{\mathrm{ns}}$ & $-.11 *$ & $-.05^{\mathrm{ns}}$ & $.05^{\mathrm{ns}}$ & $-.22 * *$ & $-.11 * *$ & $-.08^{\dddot{\imath}}$ & $-.16^{* *}$ \\
\hline \multicolumn{9}{|l|}{ Englisch } \\
\hline 5. Klasse & $-.12 * *$ & $-.09 *$ & $-.14 * *$ & $-.06^{\mathrm{ns}}$ & $-.14 * *$ & $-.12 * *$ & $-.20 * *$ & $-.15^{* *}$ \\
\hline 6. Klasse & $-.11 * *$ & $-.12 * *$ & $-.16 * *$ & $-.09^{\ddot{i}}$ & $-.27 * *$ & $-.10 *$ & $-.22 * *$ & $-.19 * *$ \\
\hline \multirow[t]{2}{*}{ Klasse } & 3. & 4. & 5. & 6. & 3. & 4. & 5. & 6. \\
\hline & \multicolumn{4}{|c|}{ Nutzungszeiten } & \multicolumn{4}{|c|}{ Inhalte } \\
\hline
\end{tabular}

Anmerkung. Kontrollierte Variablen: Wohlstandsindex, IQ (3. Klasse). * $p<.05$, ** $p<.01$, i $p<.10$.

Zur eingehenderen längsschnittlichen Überprüfung des genauen Wirkzusammenhangs exzessiv zeitlicher wie inhaltlicher Mediennutzung auf Schulnoten wurden 
drei weitere Strukturgleichungsmodelle ${ }^{105}$ berechnet, jeweils getrennt für die Wirkung der Nutzungszeit von Fernsehen und Computerspielen sowie gemeinsam für die Wirkung der Nutzung gewalthaltiger Fernseh- und Computerspielinhalte. In die Berechnungen gingen die vier hinsichtlich des Befragungstermins vergleichbaren Messzeitpunkte (Ende der dritten, vierten, fünften und sechsten Klasse) mit den Noten in den Kernfächern Deutsch, Mathematik und Sachkunde (dritte und vierte Klasse) bzw. Englisch (fünfte und sechste Klasse) ein. Eine derartige Betrachtung des Faktors Schulleistung kann als konservative Testung bezeichnet werden, da durch die Berücksichtigung des Kernfaches Mathematik zwar ein für den Faktor Schulleistung bedeutsames Fach berücksichtigt wurde, welches aber in den bisherigen Auswertungen nur einen geringen Zusammenhang mit den verschiedenen Medienvariablen aufwies. Aufgenommen in die Analysen wurden dabei nur Personen, zu denen zum dritten, vierten oder fünften Messzeitpunkt und insgesamt mindestens zu zwei Messzeitpunkten Daten vorlagen. Aufgrund unterschiedlicher Mediennutzungsgewohnheiten von Jungen und Mädchen wurde das Geschlecht der Kinder insofern berücksichtigt, als dass zusätzlich getrennte Modelle jeweils für Jungen und Mädchen berechnet wurden. In allen Modellen wurde für die Untersuchungsgruppenzugehörigkeit der Kinder kontrolliert.

105 Für die Berechnung dieses und aller weiteren Strukturgleichungsmodelle in Amos (Version 6.0, Arbuckle, 2005) mit Längsschnittdaten wurde zur Behandlung der Missing-Werte das Verfahren der full information maximum likelihood $(\mathrm{fiml})$ verwendet. Andere Verfahren der Datenimputation, wie z. B. mean substitution, wurden gänzlich verworfen, da diese Verfahren vor allem die Varianz in den Daten weiter reduzieren würden, was bei einer hohen Anzahl an Missing-Werten auch die korrelative Beziehung zwischen zwei Variablen beeinflussen würde. Das Längsschnittdesign der Studie könnte es auch nahelegen, Missing-Werte nicht über den Gruppenmittelwert, sondern über den Personenmittelwert über die Messzeitpunkte zu ersetzen, was allerdings verworfen werden muss, da dieses Verfahren zu scheinbar linearen Verläufen führen könnte. Das Verfahren der listwise deletion wurde verworfen, da dieses bei Vorhandensein bereits eines Missing-Wertes in einer der Variablen zu einem Ausschluss dieser Person aus dem Datensatz führen würde. Insbesondere bei der längsschnittlichen Betrachtung der Daten würde dieses Verfahren zu einer unverhältnismäßigen und nicht gerechtfertigten Verringerung der Stichprobe führen.

Als Stichprobenauswahl wurden in allen längsschnittlichen Modellen der Schulleistung nur Kinder der Interventions- und Kontrollklassen aufgenommen, zu denen zum zweiten, dritten, vierten oder fünften Messzeitpunkt und insgesamt mindestens zu zwei Messzeitpunkten Daten vorlagen. Die Baselineklassen wurden wegen fehlender Daten für den zweiten und dritten Messzeitpunkt komplett aus diesen Analysen ausgeschlossen.

Bei den Modellschätzungen wurden korrelierte Messfehler zwischen identischen manifesten Variablen angenommen, um einer Interkorrelation dieser über die Messzeitpunkte Rechnung zu tragen (vgl. Van Aken, Helmke \& Schneider, 1997). 
Das Modell für die Fernsehnutzungszeiten (siehe Abbildung 54), die über die Nutzungszeiten der Kinder an einem Schultag sowie am Wochenende ${ }^{106}$ erhoben wurden, zeigt zunächst bei guter Modellpassung (Gesamt: $\chi 2=174.28$ (149, $N=897), R M S E A=.01, T L I=.99, C F I=.99$; Jungen: $\chi 2=160.01(149, N=456)$, RMSEA $=.01, T L I=.99, C F I=.99$; Mädchen: $\chi 2=172.51(149, N=441)$, RMSEA $=.02, T L I=.99, C F I=.99)$ für die dritte Klasse einen statistisch bedeutsamen querschnittlichen korrelativen Zusammenhang ( $\varphi=-.14)$ zwischen höheren Fernsehnutzungszeiten und schlechteren Schulleistungen.

Dieser Zusammenhang fällt jedoch für die Jungen $(\varphi=-.20)$ deutlich größer als für die Mädchen $\left(\varphi=-.06^{\text {ns }}\right)$ aus. Über die Messzeitpunkte betrachtet, sind die Noten ${ }^{107}$ (Gesamt: $\beta_{34}=.92, \beta_{45}=.93, \beta_{56}=.92$, Jungen: $\beta_{34}=.92, \beta_{45}=.92$, $\beta_{56}=.93$, Mädchen: $\beta_{34}=.93, \beta_{45}=.95, \beta_{56}=.93$ ) das ,stabilere“ der beiden Merkmale (Fernsehnutzungszeiten; Gesamt: $\beta_{34}=.69, \beta_{45}=.74, \beta_{56}=.74$, Jungen: $\beta_{34}=.67, \beta_{45}=.68, \beta_{56}=.77$, Mädchen: $\beta_{34}=.71, \beta_{45}=.79, \beta_{56}=.71$ ), wobei die Stabilitäten für Noten und Mediennutzungszeiten über die beiden Geschlechter vergleichbar sind, einzige Ausnahme ist der Zusammenhang zwischen den Mediennutzungszeiten der vierten und fünften Klasse. Von besonderer Bedeutung sind jedoch die sechs längsschnittlichen Pfade $^{108}$ zwischen Mediennutzungszeiten und Schulnoten, da mit diesen Aussagen über eine kausale Wirkrichtung möglich sind.

Im Gesamtmodell zum Fernsehen deuten die Kreuzpfade zwischen den Messzeitpunkten bei einer insgesamt geringen Höhe (was sich in den Partialkorrelationen bereits angedeutet hatte und in hohen Stabilitäten der Schulleistungen begründet liegt) auf eine wechselnde Beeinflussung beider Variablen in zeitlicher

106 Gesamt: 3 . Klasse $-\lambda_{\text {Schultag }}=.72, \lambda_{\text {Wochenende }}=.79,4$. Klasse $-\lambda_{\text {Schultag }}=.67, \lambda_{\text {Wochenende }}=$. 85,5 . Klasse $-\lambda_{\text {Schultag }}=.75, \lambda_{\text {Wochenende }}=.85,6$. Klasse $-\lambda_{\text {Schultag }}=.76, \lambda_{\text {Wochenende }}=.89$; Jungen: 3 . Klasse $-\lambda_{\text {Schultag }}=.66, \lambda_{\text {Wochenende }}=.74,4$. Klasse $-\lambda_{\text {Schultag }}=.64, \lambda_{\text {Wochenende }}=$. 83,5 . Klasse $-\lambda_{\text {Schultag }}=.77, \lambda_{\text {Wochenende }}=.82,6$. Klasse $-\lambda_{\text {Schultag }}=.77, \lambda_{\text {Wochenende }}=.83$; Mädchen: 3 . Klasse $-\lambda_{\text {Schultag }}=.83, \lambda_{\text {Wochenende }}=.74,4$. Klasse $-\lambda_{\text {Schultag }}=.77, \lambda_{\text {Wochenen- }}$ de $=.80,5$. Klasse $-\lambda_{\text {Schultag }}=.82, \lambda_{\text {Wochenende }}=.79,6$. Klasse $-\lambda_{\text {Schultag }}=.86, \lambda_{\text {Wochenende }}=$. 84.

107 Gesamt: 3 . Klasse $-\lambda_{\text {Deutsch }}=.86, \lambda_{\text {Mathematik }}=.77, \lambda_{\text {Sachkunde }}=.76,4$. Klasse $-\lambda_{\text {Deutsch }}=.87$, $\lambda_{\text {Mathematik }}=.78, \lambda_{\text {Sachkunde }}=.83,5$. Klasse $-\lambda_{\text {Deutsch }}=.85, \lambda_{\text {Mathematik }}=.75, \lambda_{\text {Englisch }}=.80,6$. Klasse $-\lambda_{\text {Deutsch }}=.87, \lambda_{\text {Mathematik }}=.79, \lambda_{\text {Englisch }}=.87$; Jungen: 3 . Klasse $-\lambda_{\text {Deutsch }}=.86$, $\lambda_{\text {Mathematik }}=.76, \lambda_{\text {Sachkunde }}=.72,4$. Klasse $-\lambda_{\text {Deutsch }}=.87, \lambda_{\text {Mathematik }}=.80, \lambda_{\text {Sachkunde }}=.84,5$. Klasse $-\lambda_{\text {Deutsch }}=.84, \lambda_{\text {Mathematik }}=.75, \lambda_{\text {Englisch }}=.81,6$. Klasse $-\lambda_{\text {Deutsch }}=.86, \lambda_{\text {Mathematik }}=$. 79, $\lambda_{\text {Englisch }}=.88$; Mädchen: 3 . Klasse $-\lambda_{\text {Deutsch }}=.86, \lambda_{\text {Mathematik }}=.83, \lambda_{\text {Sachkunde }}=.79,4$. Klasse $-\lambda_{\text {Deutsch }}=.87, \lambda_{\text {Mathematik }}=.81, \lambda_{\text {Sachkunde }}=.81,5$. Klasse $-\lambda_{\text {Deutsch }}=.85, \lambda_{\text {Mathema- }}$ tik $=.79, \lambda_{\text {Englisch }}=.78,6$. Klasse $-\lambda_{\text {Deutsch }}=.88, \lambda_{\text {Mathematik }}=.82, \lambda_{\text {Englisch }}=.84$.

108 Diese Pfade stehen für eine Veränderung in den Schulnoten, die durch die Mediennutzung erklärt werden können sowie für eine Veränderung in den Mediennutzungszeiten, die durch die Schulnoten erklärt werden kann. Angesichts der hohen Stabilitäten zwischen den Schulnoten der verschiedenen Messzeitpunkte bleibt hier allerdings nur ein geringer Spielraum auf Seiten der Schulleistungen. Bezüglich der Mediennutzungszeiten steht hier deutlich mehr unerklärte Varianz zur Verfügung. 
Abfolge hin, d. h. Kinder mit schlechteren Noten schauen zunächst verstärkt Fernsehprogramme, was in den späteren Jahren zu schlechteren Noten führt; oder, Kinder mit besseren Noten schauen zunächst weniger fern, was in den späteren Jahren wiederum zu besseren Noten führt. Ab der vierten Klasse tragen die Schulleistungen allerdings nicht mehr zur Erklärung von Unterschieden in den Fernsehnutzungszeiten bei; hier müssen andere Einflussfaktoren eine Rolle spielen (vgl. Abbildung 47). Der größte Pfad, insgesamt sowie für beide Geschlechter getrennt, ist dabei von den Schulnoten der dritten Klasse auf die Fernsehnutzungszeiten der vierten Klasse $\left(\beta_{\text {Gesamt }}=-.14, \beta_{\text {Jungen }}=-.14, \beta_{\text {Mädchen }}=-.15\right) \mathrm{zu}$ beobachten. Die beiden (geringeren, jedoch) statistisch bedeutsamen Pfade von Fernsehnutzungszeiten der vierten und fünften Klasse auf die Schulnoten der fünften und sechsten Klasse des Gesamtmodells $\left(\beta_{45 \text {, Gesamt }}=-.05, \beta_{56 \text {, Gesamt }}=-\right.$. $05)$ sind bei einer nach Geschlecht getrennten Betrachtung lediglich für die Jungen in der vierten Klasse noch von Bedeutung $\left(\beta_{45}\right.$, Jungen $=-.08, \beta_{56 \text {, Jungen }}=-$. $\left.04^{\mathrm{ns}}\right)$. Zusammengefasst wird das Muster des Gesamtmodells somit für beide Geschlechter getrennt nur zum Teil abgebildet. So kann zwar für die Jungen eine wechselnde Beeinflussung in zeitlicher Abfolge bestätigt werden, das Modell der Mädchen würde allerdings lediglich nahelegen, dass Kinder mit schlechteren Noten verstärkt Fernsehprogramme schauen (ohne eine Wirkung in die andere Richtung). 


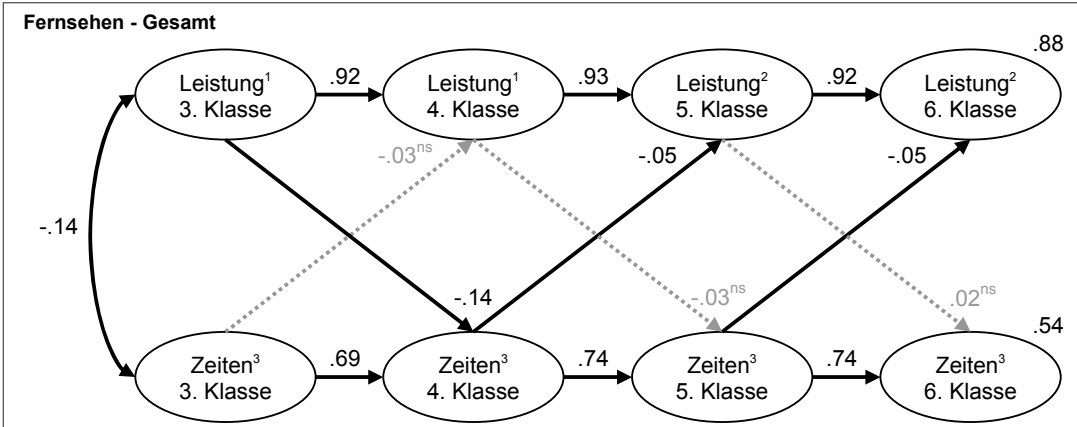

Modell Fit:

$X^{2}=174.28, \mathrm{df}=149, \mathrm{TLI}=.99, \mathrm{CFI}=.99, \mathrm{RMSEA}=.01$, Standardisierte Parameter; alle Pfade mindestens $p \leq .05$ (falls nicht anders gekennzeichnet).

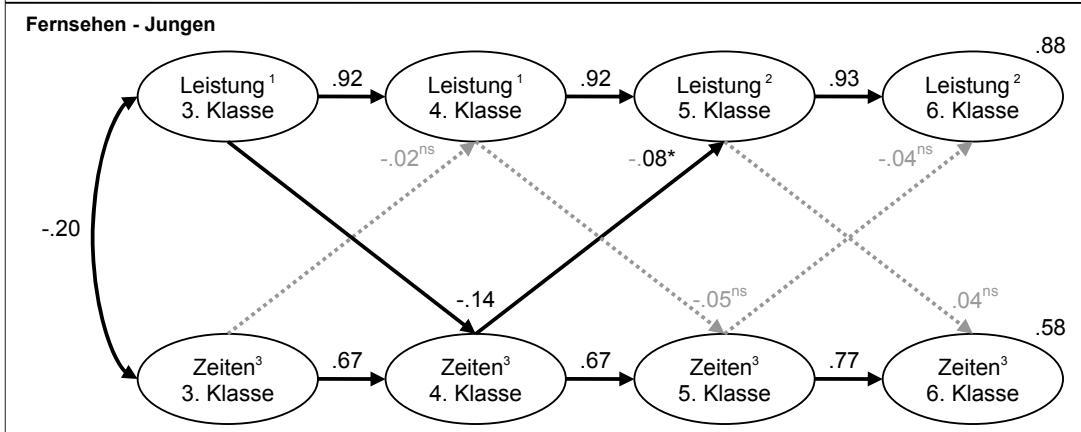

Modell Fit:

$\mathrm{X}^{2}=160.01, \mathrm{df}=149, \mathrm{TLI}=.99, \mathrm{CFI}=.99, \mathrm{RMSEA}=.01$, Standardisierte Parameter; alle Pfade mindestens $p \leq .05$ (falls nicht anders gekennzeichnet). ${ }^{*} p<.10$

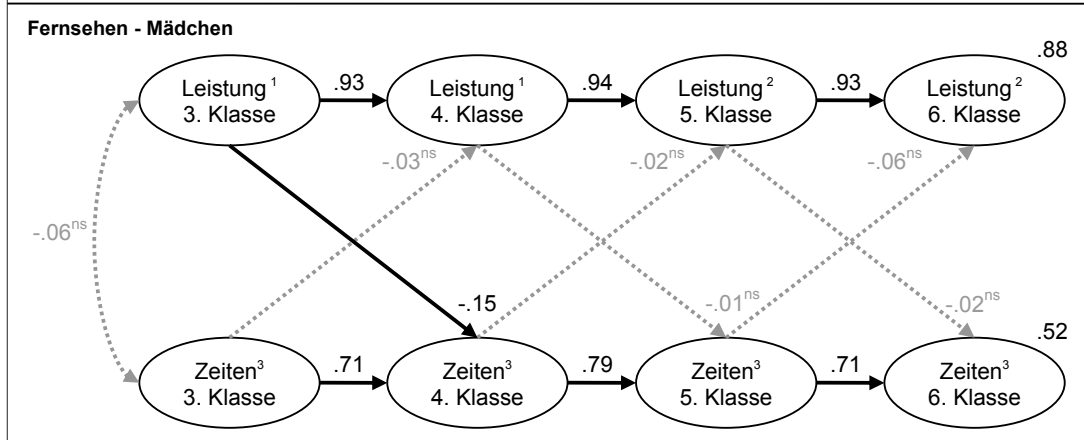

$\mathrm{X}^{2}=172.51, \mathrm{df}=149, \mathrm{TLI}=.99, \mathrm{CFI}=.99, \mathrm{RMSEA}=.02$, Standardisierte Parameter; alle Pfade mindestens $p \leq .05$ (falls nicht anders gekennzeichnet).

${ }^{1}$ Noten in Deutsch, Mathematik und Sachkunde.

${ }^{2}$ Noten in Deutsch, Mathematik und Englisch.

${ }^{3}$ Schätzfrage im Einstundenabstand getrennt für Wochentags und am Wochenende

Abbildung 54. Strukturgleichungsmodelle zum längsschnittlichen Zusammenhang von Fernsehnutzungszeiten und Schulnoten. 
Das Modell für die Computerspielnutzungszeiten (siehe Abbildung 55), die erneut über die Nutzungszeiten der Kinder an einem Schultag sowie am Wochenende ${ }^{109}$ erhoben wurden, zeigt bei guter Modellpassung (Gesamt: $\chi 2=271.42(148, N=897)$, RMSEA $=.03, T L I=.98, C F I=.99$; Jungen: $\chi 2=175.05(148, N=456), R M S E A=.02, T L I=.99, C F I=.99$; Mädchen: $\chi 2=230.49(148, N=441), R M S E A=.04, T L I=.97, C F I=.98)$ für die dritte Klasse einen statistisch bedeutsamen querschnittlichen korrelativen Zusammenhang $(\varphi=-.26)$ zwischen höheren Computerspielzeitenzeiten und schlechteren Schulleistungen. ${ }^{110}$ Dieser Zusammenhang ist im Vergleich zu den Fernsehnutzungszeiten - was in den Partialkorrelationen aus Tabelle 51 bereits ersichtlich wurde - stärker ausgeprägt. Zudem ist er bei den Jungen $(\varphi=-.29)$ etwas größer als bei den Mädchen $(\varphi=-.23)$. Die Stabilitäten der Noten sind mit denen aus Abbildung 54 vergleichbar (Gesamt: $\beta_{34}=.90, \beta_{45}=.92, \beta_{56}=.93$, Jungen: $\beta_{34}=$. 90, $\beta_{45}=.91, \beta_{56}=.94$, Mädchen: $\beta_{34}=.92, \beta_{45}=.95, \beta_{56}=.93$ ), die der Nutzungszeiten (Gesamt: $\beta_{34}=.59, \beta_{45}=.64, \beta_{56}=.60$, Jungen: $\beta_{34}=.54, \beta_{45}=.63, \beta_{56}=.69$, Mädchen: $\beta_{34}=.48, \beta_{45}=.53, \beta_{56}=.40$ ) liegen auf einem geringeren Niveau.

Im Gesamtmodell für Computerspiele deuten die Kreuzpfade zwischen den Messzeitpunkten ebenfalls auf eine wechselnde Beeinflussung beider Variablen in zeitlicher Abfolge hin, allerdings im Vergleich zu den Fernsehnutzungszeiten in umgekehrter Reihenfolge. Das heißt hohe Computerspielzeiten in der dritten und vierten Klasse können zunächst Verschlechterungen in den Schulleistungen der vierten und fünften Klasse erklären $\left(\beta_{34 \text {, Gesamt }}=-.07, \beta_{45 \text {, Gesamt }}=-.08\right)$, letztere können dann erhöhte Computerspielzeiten in der sechsten Klasse $\left(\beta_{56}\right.$, Gesamt $=-.13$ ) erklären. In der dritten und vierten Klasse tragen die Schulleistungen nicht zur Erklärung von Unterschieden in den Computerspielzeiten bei. Dieses Muster des Gesamtmodells findet sich bei einer nach Geschlecht getrennten Betrachtung allerdings nur zum Teil wieder. So entfällt für die Jungen der statistisch bedeutsame Pfad von den Schulleistungen der fünften auf die Computer-

109 Gesamt: 3 . Klasse $-\lambda_{\text {Schultag }}=.76, \lambda_{\text {Wochenende }}=.78,4$. Klasse $-\lambda_{\text {Schultag }}=.73, \lambda_{\text {Wochenende }}=$. 81,5 . Klasse $-\lambda_{\text {Schultag }}=.74, \lambda_{\text {Wochenende }}=.92,6$. Klasse $-\lambda_{\text {Schultag }}=.78, \lambda_{\text {Wochenende }}=.90 ;$ Jungen: 3 . Klasse $-\lambda_{\text {Schultag }}=.70, \lambda_{\text {Wochenende }}=.82,4$. Klasse $-\lambda_{\text {Schultag }}=.70, \lambda_{\text {Wochenende }}=$. 78,5 . Klasse $-\lambda_{\text {Schultag }}=.72, \lambda_{\text {Wochenende }}=.88,6$. Klasse $-\lambda_{\text {Schultag }}=.72, \lambda_{\text {Wochenende }}=.87$; Mädchen: 3 . Klasse $-\lambda_{\text {Schultag }}=.81, \lambda_{\text {Wochenende }}=.69,4$. Klasse $-\lambda_{\text {Schultag }}=.77, \lambda_{\text {Wochenen- }}$ $\underset{\mathrm{de}}{=.75,5}$. Klasse $-\lambda_{\text {Schultag }}=.81, \lambda_{\text {Wochenende }}=.91,6$. Klasse $-\lambda_{\text {Schultag }}=.82, \lambda_{\text {Wochenende }}=$. 90 .

110 Gesamt: 3 . Klasse $-\lambda_{\text {Deutsch }}=.87, \lambda_{\text {Mathematik }}=.76, \lambda_{\text {Sachkunde }}=.76,4$. Klasse $-\lambda_{\text {Deutsch }}=.88$, $\lambda_{\text {Mathematik }}=.77, \lambda_{\text {Sachkunde }}=.83,5$. Klasse $-\lambda_{\text {Deutsch }}=.85, \lambda_{\text {Mathematik }}=.74, \lambda_{\text {Englisch }}=.80,6$. Klasse $-\lambda_{\text {Deutsch }}=.88, \lambda_{\text {Mathematik }}=.78, \lambda_{\text {Englisch }}=.87$; Jungen: 3 . Klasse $-\lambda_{\text {Deutsch }}=.86$, $\lambda_{\text {Mathematik }}=.76, \lambda_{\text {Sachkunde }}=.72,4$. Klasse $-\lambda_{\text {Deutsch }}=.87, \lambda_{\text {Mathematik }}=.80, \lambda_{\text {Sachkunde }}=.84,5$. Klasse $-\lambda_{\text {Deutsch }}=.84, \lambda_{\text {Mathematik }}=.75, \lambda_{\text {Englisch }}=.81,6$. Klasse $-\lambda_{\text {Deutsch }}=.86, \lambda_{\text {Mathematik }}=$. 79, $\lambda_{\text {Englisch }}=.88$; Mädchen: 3 . Klasse $-\lambda_{\text {Deutsch }}=.86, \lambda_{\text {Mathematik }}=.83, \lambda_{\text {Sachkunde }}=.80,4$. Klasse $-\lambda_{\text {Deutsch }}=.87, \lambda_{\text {Mathematik }}=.80, \lambda_{\text {Sachkunde }}=.81,5$. Klasse $-\lambda_{\text {Deutsch }}=.86, \lambda_{\text {Mathema- }}$ tik $=.79, \lambda_{\text {Englisch }}=.78,6$. Klasse $-\lambda_{\text {Deutsch }}=.89, \lambda_{\text {Mathematik }}=.81, \lambda_{\text {Englisch }}=.84$. 
spielzeiten der sechsten Klasse $\left(\beta_{56, \text { Jungen }}=-.05^{\text {ns }}\right)$, und zusätzlich ist für die Jungen in der dritten Klasse eine Wechselwirkung beider Variablen zu beobachten $\left(\beta_{34}\right.$, Jungen, Leistung-Zeiten $\left.=-.11, \beta_{34 \text {, Jungen, Zeiten-Leistung }}=-.08\right)$. Das Modell der Mädchen würde allerdings wiederum nahelegen, dass Kinder mit schlechteren Noten lediglich verstärkt Zeit vor dem Computer verbringen um zu Spielen $\left(\beta_{45 \text {, Gesamt }}=\right.$ $-.10, \beta_{56 \text {, Gesamt }}=-.18$ ), dies sich in den folgenden Jahren aber nicht negativ auf die Schulleistungen auswirkt. 


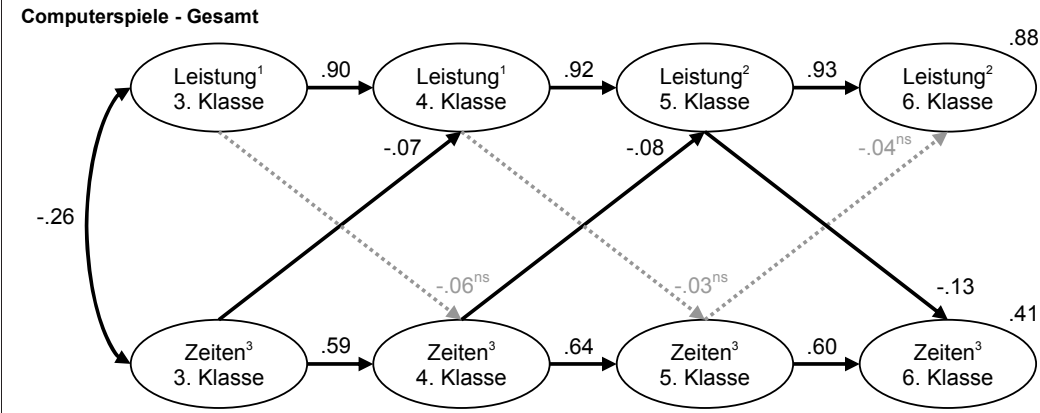

Modell Fit:

$\mathrm{X}^{2}=271.42, \mathrm{df}=148, \mathrm{TLI}=.98, \mathrm{CFI}=.99, \mathrm{RMSEA}=.03$, Standardisierte Parameter; alle Pfade mindestens $p \leq .05$ (falls nicht anders gekennzeichnet)

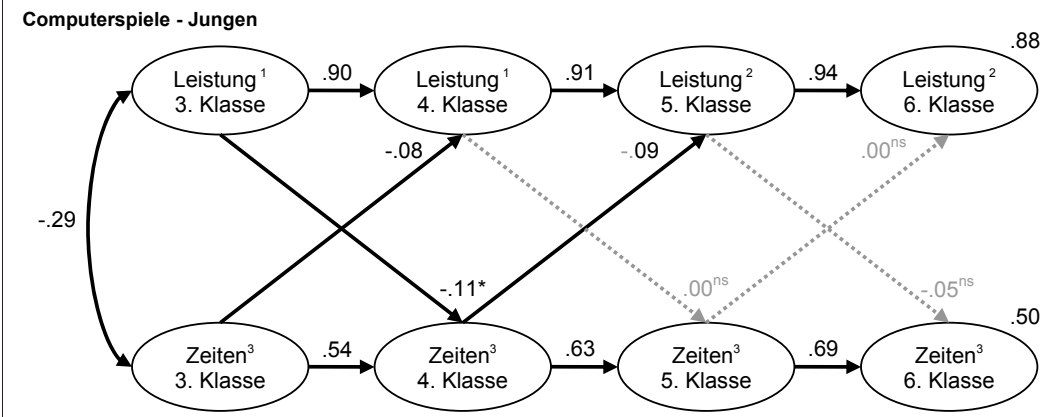

Modell Fit:

$X^{2}=175.05, \mathrm{df}=148, \mathrm{TLI}=.99, \mathrm{CFI}=.99, \mathrm{RMSEA}=.02$, Standardisierte Parameter; alle Pfade mindestens $p \leq .05$ (falls nicht anders gekennzeichnet). ${ }^{*} p<.10$

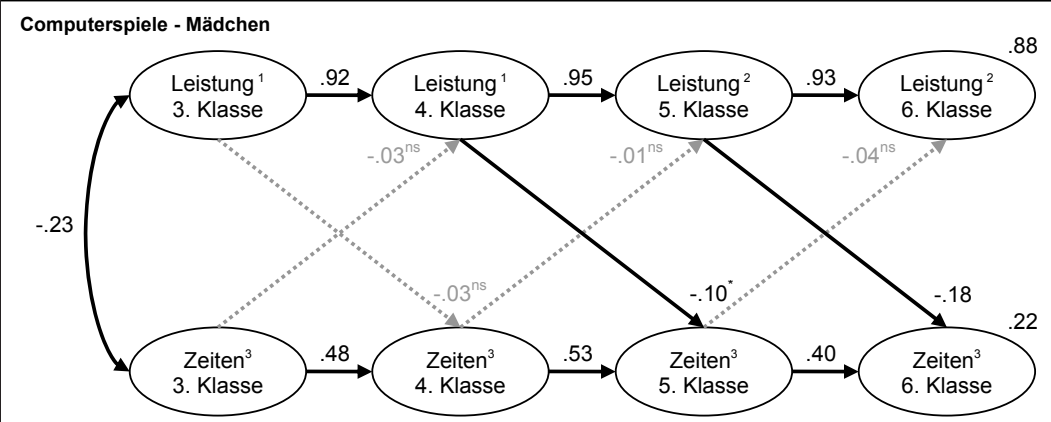

Modell Fit:

$X^{2}=230.49, \mathrm{df}=148, \mathrm{TLI}=.97, \mathrm{CFI}=.98, \mathrm{RMSEA}=.04$, Standardisierte Parameter; alle Pfade mindestens $p \leq .05$ (falls nicht anders gekennzeichnet). ${ }^{*} p<.10$

${ }^{1}$ Noten in Deutsch, Mathematik und Sachkunde.

${ }^{2}$ Noten in Deutsch, Mathematik und Englisch.

${ }^{3}$ Schätzfrage im Einstundenabstand getrennt für Wochentags und am Wochenende

Abbildung 55. Strukturgleichungsmodelle zum längsschnittlichen Zusammenhang von Computerspielnutzungszeiten und Schulnoten. 
Das Modell für die Inhalte (siehe Abbildung 56), die über den Konsum von Filmen und/oder Computerspielen ab 16 bzw. 18 Jahren erhoben wurden, ${ }^{111}$ zeigt zunächst bei guter Modellpassung (Gesamt: $\chi 2=261.49(149, N=897)$, RMSEA $=.03$, TLI $=.98, C F I=.99$; Jungen: $\chi 2=178.65(148, N=456)$, RMSEA $=.02, T L I=.99, C F I=.99$; Mädchen: $\chi 2=209.12(151, N=441)$, RMSEA $=.03, T L I=.98, C F I=.99)$ für die dritte Klasse einen statistisch bedeutsamen querschnittlichen korrelativen Zusammenhang $(\varphi=-.26)$ zwischen dem Konsum gewalthaltiger Medieninhalte und schlechteren Schulleistungen, ${ }^{112}$ der mit dem der Computerspielzeiten aus Abbildung 55 vergleichbar ist. Im Gegensatz zu den Computerspielzeiten ist dieser Zusammenhang für die Jungen $(\varphi=-$. 37) deutlich größer als für die Mädchen $(\varphi=-.15)$. Die Stabilitäten der Noten sind mit erneut denen aus Abbildung 54 und Abbildung 55 vergleichbar (Gesamt: $\beta_{34}=.90, \beta_{45}=.91, \beta_{56}=.93$, Jungen: $\beta_{34}=.89, \beta_{45}=.93, \beta_{56}=.94$, Mädchen: $\left.\beta_{34}=.92, \beta_{45}=.94, \beta_{56}=.94\right)$. Der Konsum gewalthaltiger Medieninhalte (Gesamt: $\beta_{34}=.80, \beta_{45}=.87, \beta_{56}=.73$, Jungen: $\beta_{34}=.77, \beta_{45}=.79, \beta_{56}=.59$, Mädchen: $\beta_{34}=.74, \beta_{45}=.88, \beta_{56}=.76$ ) verändert sich über die Zeit jedoch deutlich weniger als die zuvor dargestellten Mediennutzungszeiten.

Die Kreuzpfade zwischen den Messzeitpunkten des Gesamtmodells für die Inhalte sind vergleichbar mit denen des Modells der Computerspielzeiten, d. h. es kann eine entsprechende wechselnde Beeinflussung beider Variablen in zeitlicher Abfolge beobachtet werden. Der erhöhte Konsum gewalthaltiger Medieninhalte in der dritten und vierten Klasse kann zunächst Verschlechterungen in den Schulleistungen der vierten und fünften Klasse erklären $\left(\beta_{34 \text {, Gesamt }}=-.08, \beta_{45 \text {, Ge- }}\right.$ samt $=-.09)$, letztere können dann den erhöhten Konsum gewalthaltiger Medieninhalte in der sechsten Klasse $\left(\beta_{56, \text { Gesamt }}=-.15\right)$ erklären. In der dritten und vierten Klasse tragen die Schulleistungen wiederum nicht zur Erklärung von Unterschieden in der Gewaltmediennutzung bei. Diese zeitliche Abfolge ist jedoch bei einer nach Geschlecht getrennten Betrachtung nur noch in Ausschnitten zu beobachten. So entfällt für die Jungen der statistisch bedeutsame Pfad vom Konsum ge-

111 Gesamt: 3 . Klasse $-\lambda_{\text {fab } 1618}=.63, \lambda_{\text {cab1618 }}=.71,4$. Klasse $-\lambda_{\text {fab1618 }}=.64, \lambda_{\text {cab1618 }}=.70,5$. Klasse $-\lambda_{\text {fab1618 }}=.64, \lambda_{\text {cab1618 }}=.70,6$. Klasse $-\lambda_{\text {fab1618 }}=.60, \lambda_{\text {cab1618 }}=.74$; Jungen: 3 . Klasse $-\lambda_{\text {fab1618 }}=.76, \lambda_{\text {cab1618 }}=.68,4$. Klasse $-\lambda_{\text {fab1618 }}=.70, \lambda_{\text {cab1618 }}=.72,5$. Klasse $\lambda_{\text {fab1618 }}=.68, \lambda_{\text {cab1618 }}=.75,6$. Klasse $-\lambda_{\text {fab1618 }}=.57, \lambda_{\text {cab1618 }}=.70$; Mädchen: 3 . Klasse $\lambda_{\text {fab } 1618}=.60, \lambda_{\text {cab } 1618}=.59,4$. Klasse $-\lambda_{\text {fab } 1618}=.61, \lambda_{\text {cab } 1618}=.50,5$. Klasse $-\lambda_{\text {fab } 1618}=.63$, $\lambda_{\text {cab1618 }}=.46,6$. Klasse $-\lambda_{\text {fab1618 }}=.61, \lambda_{\text {cab1618 }}=.65$.

112 Gesamt: 3 . Klasse $-\lambda_{\text {Deutsch }}=.87, \lambda_{\text {Mathematik }}=.76, \lambda_{\text {Sachkunde }}=.76,4$. Klasse $-\lambda_{\text {Deutsch }}=.88$, $\lambda_{\text {Mathematik }}=.78, \lambda_{\text {Sachkunde }}=.83,5$. Klasse $-\lambda_{\text {Deutsch }}=.85, \lambda_{\text {Mathematik }}=.75, \lambda_{\text {Englisch }}=.80,6$. Klasse $-\lambda_{\text {Deutsch }}=.87, \lambda_{\text {Mathematik }}=.79, \lambda_{\text {Englisch }}=.87$; Jungen: 3 . Klasse $-\lambda_{\text {Deutsch }}=.86$, $\lambda_{\text {Mathematik }}=.76, \lambda_{\text {Sachkunde }}=.72,4$. Klasse $-\lambda_{\text {Deutsch }}=.87, \lambda_{\text {Mathematik }}=.80, \lambda_{\text {Sachkunde }}=.84,5$. Klasse $-\lambda_{\text {Deutsch }}=.84, \lambda_{\text {Mathematik }}=.75, \lambda_{\text {Englisch }}=.81,6$. Klasse $-\lambda_{\text {Deutsch }}=.86, \lambda_{\text {Mathematik }}=$. 79, $\lambda_{\text {Englisch }}=.88$; Mädchen: 3 . Klasse $-\lambda_{\text {Deutsch }}=.86, \lambda_{\text {Mathematik }}=.83, \lambda_{\text {Sachkunde }}=.80,4$. Klasse $-\lambda_{\text {Deutsch }}=.87, \lambda_{\text {Mathematik }}=.81, \lambda_{\text {Sachkunde }}=.81,5$. Klasse $-\lambda_{\text {Deutsch }}=.86, \lambda_{\text {Mathema- }}$ tik $=.79, \lambda_{\text {Englisch }}=.78,6$. Klasse $-\lambda_{\text {Deutsch }}=.88, \lambda_{\text {Mathematik }}=.82, \lambda_{\text {Englisch }}=.84$. 
walthaltiger Medieninhalte der vierten auf die Computerspielzeiten der fünften Klasse $\left(\beta_{45}\right.$, Jungen $\left.=-.02^{\text {ns }}\right)$ und für die Mädchen der statistisch bedeutsame Pfad vom Konsum gewalthaltiger Medieninhalte der dritten auf die Computerspielzeiten der vierten Klasse $\left(\beta_{34, \text { Mädchen }}=-.06^{\mathrm{ns}}\right)$. Für beide Geschlechter bestätigt sich aber somit der Verlauf vom Konsum gewalthaltiger Medieninhalte über schlechtere Schulleistungen hin zum erhöhten Konsum gewalthaltiger Medieninhalte. 


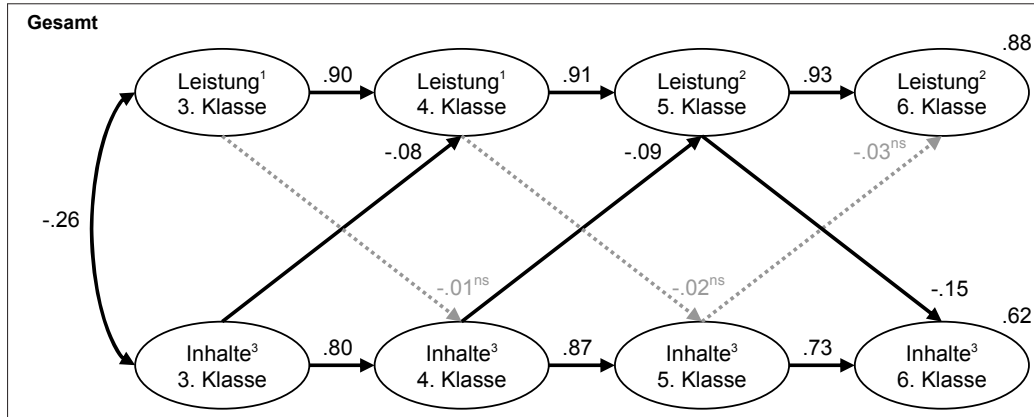

Modell Fit:

$\mathrm{X}^{2}=261.49 \mathrm{df}=149, \mathrm{TLI}=.98, \mathrm{CFI}=.99, \mathrm{RMSEA}=.03$, Standardisierte Parameter; alle Pfade mindestens $p \leq .05$ (falls nicht anders gekennzeichnet).

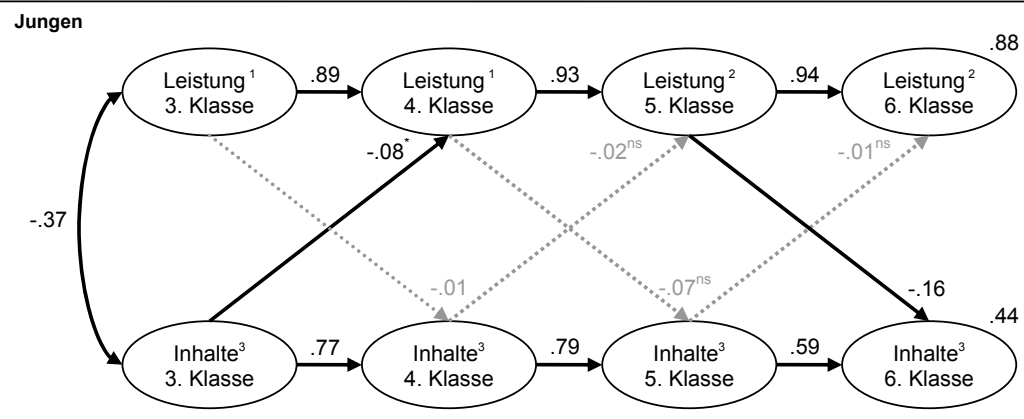

Modell Fit:

$\mathrm{X}^{2}=178.65, \mathrm{df}=148, \mathrm{TLI}=.99, \mathrm{CFI}=.99, \mathrm{RMSEA}=.02$, Standardisierte Parameter; alle Pfade mindestens $p \leq .05$ (falls nicht anders gekennzeichnet). ${ }^{*} p<.10$

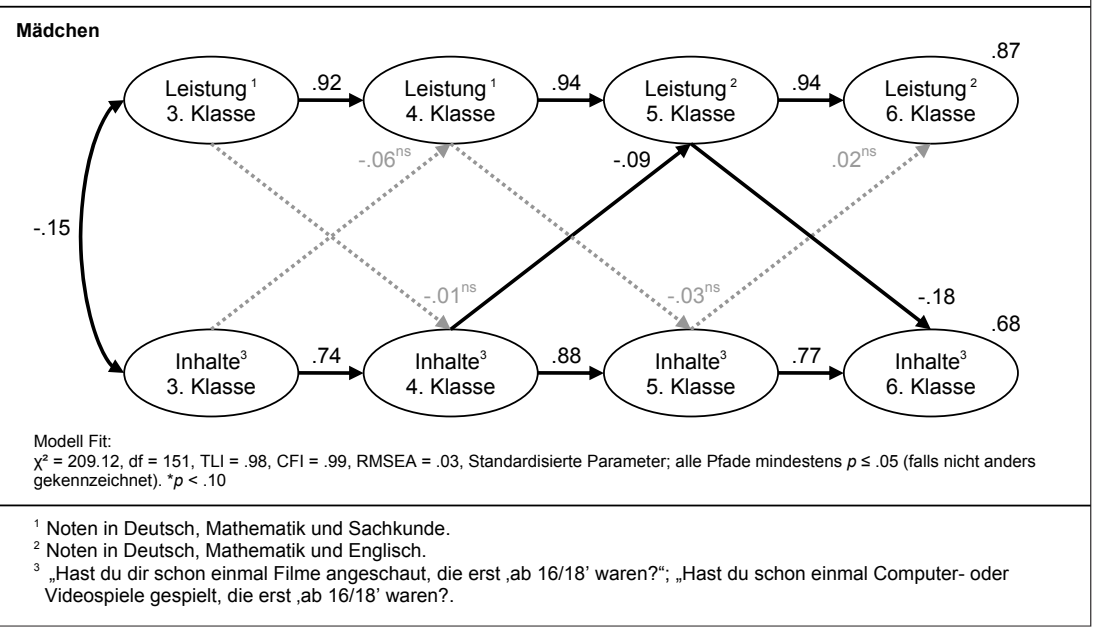

Abbildung 56. Strukturgleichungsmodelle zum längsschnittlichen

Zusammenhang der Nutzung altersinadäquater Computerspiele und Fernsehinhalte und Schulnoten. 
Zusammengefasst weisen die quer- und längsschnittlichen Befunde auf eine Wechselwirkung zwischen einer inhaltlich problematischen wie zeitlich exzessiven Mediennutzung und schlechten Schulleistungen hin. Die berechneten Strukturgleichungsmodelle legen bei Betrachtung der Gesamtmodelle dabei eine wechselnde Beeinflussung in zeitlicher Abfolge nahe, wobei mit Ausnahme der Fernsehzeiten der „Start“ jeweils auf Seiten der Medienvariablen zu beobachten ist. Bei einer Unterscheidung nach Geschlecht zeigt sich jedoch, dass diese Gesamtmodelle zwar größtenteils auf die Jungen übertragen werden können, für die Mädchen aber was die Nutzungszeiten von Fernsehen und Computerspielen betrifft, eher eine einseitige Selektion in dem Sinne, dass vor allem Mädchen, die in der Schule schlechter abschneiden, in den folgenden Jahren besonders lange Medien konsumieren, anzunehmen ist.

Die Bedeutung früher kindlicher Gewaltmediennutzung. Insbesondere in den berechneten Partialkorrelationen zwischen Mediennutzungsvariablen und Schulleistungen (vgl. Tabelle 51) sowie den Modellen zur Bedeutung von Computerspielzeiten (vgl. Abbildung 55) und der Nutzung gewalthaltiger Medieninhalte (vgl. Abbildung 56) auf Schulleistungen wird zudem eine verstärkende Wirkung früher exzessiver Mediennutzung auf schlechtere Schulleistungen deutlich. Zur genaueren Einordnung der Vorhersagekraft einer frühkindlichen Mediennutzung auf späteren Schulerfolg - im Zusammenspiel weiterer bedeutsamer Einflussfaktoren (vgl. Abbildung 51) -, wurde abschließend eine Regression mit dem Kriterium mittlere Schulleistung (6. Klasse) berechnet (vgl. Tabelle 52). In diese gingen neben den Mediennutzungsvariablen, das Geschlecht der Kinder, das Bildungsniveau, der IQ der Kinder sowie die wahrgenommene elterliche $\mathrm{Zu}$ wendung als Prädiktoren ein.

In ein erstes Regressionsmodell (Modell I) wurden zunächst neben den Variablen des sozio-kulturellen Kapitals sowie des Geschlechts und des IQ der Kinder sämtliche Variablen der sechsten Klasse als Prädiktoren in die Analyse mit aufgenommen. Wie im Querschnittsmodell (vgl. Abbildung 51) tragen die unterschiedlichen Variablen in vergleichbarem Ausmaß zur Vorhersage bei. So sind das Selbstkonzept eigener Schulfähigkeit $(\beta=-.56)$ sowie der IQ der Kinder $(\beta=$ -.21) die beiden bedeutendsten Variablen zur Erklärung von Schulleistungen. Das Geschlecht der Kinder trägt jedoch im Unterschied zum Querschnittsmodell bedeutsam zur Erklärung von Schulleistungsunterschieden bei $(\beta=-.09)$. Zusätzlich ist auf Seiten des sozio-kulturellen Kapitals das Bildungsniveau $\left(\beta_{\text {niedrig }}=\right.$. $\left.16, \beta_{\text {mittel }}=.13\right)$ zur Vorhersage späterer Schulleistungen wesentlich. In die folgenden Modelle (Modell II - Modell IV) wurden jeweils die Variablen der späteren Messzeitpunkte (fünfte Klasse - Modell II, vierte Klasse - Modell III, dritte Klasse - Modell IV) in das Regressionsmodell mit aufgenommen. Erwartungsgemäß nimmt mit zunehmender Dauer zwischen den erklärenden Variablen und 
Tabelle 52. Regression Schulleistung (6. Klasse).

\begin{tabular}{ccccc}
\hline & Modell I & Modell II & Modell III & Modell IV \\
\hline Geschlecht & $-.09^{* *}$ & $-.08^{*}$ & $-.21^{* *}$ & $-.19^{* *}$ \\
Bildungsniveau & & & & \\
niedrig & $.16^{* *}$ & $.11^{* *}$ & $.18^{* *}$ & $.18^{* *}$ \\
mittel & $.13^{* *}$ & $.11^{* *}$ & $.16^{* *}$ & $.14^{* *}$ \\
hoch & Referenz & Referenz & Referenz & Referenz \\
IQ (CFT 20) & $-.21^{* *}$ & $-.23^{* *}$ & $-.29^{* *}$ & $-.29^{* *}$
\end{tabular}

\section{Klasse}

$\begin{array}{lc}\text { Selbstkonzept } & -.56^{* *} \\ \text { Elterliche Zuwendung } & .06^{\natural} \\ \text { Gewaltmediennutzung } & .05^{\text {ns }}\end{array}$

\section{Klasse}

Selbstkonzept

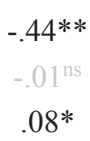

Elterliche Zuwendung

Gewaltmediennutzung

\section{Klasse}

Selbstkonzept

Elterliche Zuwendung

Gewaltmediennutzung

\section{Klasse}

Selbstkonzept

Elterliche Zuwendung

Gewaltmediennutzung

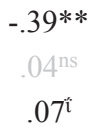

\begin{tabular}{lcccc}
\hline Korrigiertes $\boldsymbol{R 2}$ & .52 & .43 & .40 & .37 \\
$\boldsymbol{p}$ & $<.001$ & $<.001$ & $<.001$ & $<.001$ \\
\hline
\end{tabular}

Anmerkung. Abhängige Variable - Mittlere Noten: Deutsch, Mathematik, Englisch. Keine Kollinearität gegeben (alle $r \leq .32$, Toleranz $\geq .77, V I F \leq 1.3$, keinen auffälligen $C I$ ). Als Stichprobenauswahl wurden nur Kinder der Interventions- und Kontrollklassen aufgenommen, zu denen zum zweiten, dritten, vierten oder fünften Messzeitpunkt und insgesamt mindestens zu zwei Messzeitpunkten Daten vorlagen $(n=897)$. Die Baselineklassen wurden wegen fehlender Daten für den zweiten und dritten Messzeitpunkt komplett aus diesen Analysen ausgeschlossen. Bildungsniveau: Das formale Bildungsniveau ist niedrig, wenn der höchste Bildungsabschluss beider Elternteile ein Hauptschulabschluss ist; Es ist mittel, wenn der höchste Bildungsabschluss mindestens eines Elternteils die Mittlere Reife ist; Es ist hoch, wenn der höchste Bildungsabschluss mindestens eines Elternteils das Abitur oder ein abgeschlossenes Studium ist. Selbstkonzept: Selbstkonzept eigener Schulfähigkeiten (FEESS 3-4, Rauer \& Schuck, 2003). Elterliche Zuwendung: Elterliches Erziehungs- und Unterstützungsverhalten (vgl. Kapitel 1.5.1, Tabelle 14). Gewaltmediennutzung: Hat schon einmal Filme und/oder Computerspiele ab 16/18 gesehen oder gespielt (dichotome Variable). * $p<.05$, ** $p<.01$. i $^{*}<<.10$. 
der zu erklärenden Variable (mittlere Schulleistung 6. Klasse) die Erklärungskraft der einzelnen Variablen ab. Auffällig ist, dass zum einen das elterliche Erziehungs- und Unterstützungsverhalten an Bedeutung verliert und zum anderen das Selbstkonzept eigener Schulfähigkeiten zwar auch an Bedeutung verliert, aber immer noch substanziell zur Erklärung späterer Schulleistungen beiträgt. Auf der anderen Seite gewinnen bei Berücksichtigung der erklärenden Variablen vorangegangener Messungen das Geschlecht sowie der IQ der Kinder an Bedeutung zur Erklärung schulischer Leistungen. Der auffälligste Befund ist jedoch hinsichtlich der frühkindlichen Gewaltmediennutzung zu beobachten. War dieser in Model I (6. Klasse) bei Erklärung schulischer Leistungen durch Variablen desselben Messzeitpunktes noch nicht von Bedeutung, trägt der frühkindliche Konsum gewalthaltiger Medieninhalte $(\beta=.16)$ in Modell IV (3. Klasse) bedeutsam in vergleichbarer Größenordnung zum Bildungsniveau im Elternhaus $\left(\beta_{\text {nied- }}\right.$ ${ }_{\text {rig }}=.18, \beta_{\text {mittel }}=.14$ ) zur Erklärung späterer schulischer Leistungen bei.

\subsection{Diskussion}

In Kapitel 3.2 wurden zum Thema Mediennutzung und Schulleistung die folgenden beiden Leitfragen, die mit dem Berliner Längsschnitt Medien beantwortet werden sollten, formuliert:

1. Lassen sich die in der KFN-Schülerbefragung 2005 sowie in weiteren nationalen wie internationalen (quer- wie längsschnittlichen) Studien berichteten Zusammenhänge zwischen einer inhaltlich problematischen sowie einer zeitlich exzessiven Mediennutzung und schlechteren Schulleistungen bei Berücksichtigung weiterer aus der Literatur bekannter Einflussvariablen auch für die Berliner Grundschülerpopulation bestätigen?

2. Können Befunde hinsichtlich einer Wirkungsvermutung (problematische Mediennutzung führt zu schlechteren Schulleistungen) im Gegensatz zu einer Selektion (schlechtere Schülerinnen und Schüler nutzen vermehrt Medien) im Längsschnitt bestätigt werden?

Im Folgenden sollen die beiden Fragenkomplexe einzeln aufgegriffen und mit der bestehenden Forschungslage in Beziehung gesetzt werden.

Zu 1.) Betrachtung des Zusammenhangs Mediennutzung und Schulleistung unter Berücksichtigung von Drittvariablen. Die Querschnittsbefunde aus Kapitel 3.2.2 und 3.2.3 zeigten unter Berücksichtigung weiterer aus der Literatur bekannter Einflussvariablen, d. h. dem sozialen und kulturellen Kapital im Elternhaus, dem elterlichen Erziehungs- und Unterstützungsverhalten, den kognitiven Fähigkeiten des Kindes, dem Selbstkonzept eigener Fähigkeiten sowie der Schuleinstellung deutliche Zusammenhänge zwischen einer inhaltlich problema- 
tischen (wenn nach Geschlecht getrennt wurde) sowie zeitlich exzessiven Mediennutzung und Schulnoten im Kindesalter. Dieser Befund wurde bereits in ähnlicher Form in der KFN-Schülerbefragung 2005 für eine Stichprobe von durchschnittlich 10-jährigen Kindern berichtet (vgl. Mößle et al., 2007) und konnte somit bestätigt werden. Ähnliche Ergebnisse zeigten auch weitere nationale wie internationale Studien (vgl. Anderson, D. R. et al., 2001; Roe et al., 2001; Schiffer et al., 2002; Ennemoser, 2003; Gentile et al., 2004; Borzekowski \& Robinson, 2005; Hancox et al., 2005; Zimmerman \& Christakis, 2005; Sharif \& Sargent, 2006; Ennemoser \& Schneider, 2007; Johnson et al., 2007; Mößle et al., 2007; Ennemoser \& Schneider, 2009; Hastings, E. C. et al., 2009; Jaruratanasirikul et al., 2009; Skoric et al., 2009; Schittenhelm et al., 2010; Sharif et al., 2010; Baier \& Pfeiffer, 2011). In diesen Studien wurden aber die Leistungen im schulischen Bereich z. T. über andere Variablen operationalisiert und die unterschiedlichen Determinanten schulischer Leistungen (vgl. Abbildung 48) wurden selten so umfassend berücksichtigt wie in der vorliegenden Arbeit (vgl. Gentile et al., 2004; Johnson et al., 2007; Mößle et al., 2007).

Hinsichtlich der erhobenen abhängigen Variablen zur Schulleistung zeigten sich zunächst im Berliner Längsschnitt Medien, wie schon an anderer Stelle für Grundschülerinnen und Grundschüler berichtet (vgl. Mößle et al., 2007), die geschlechtsspezifischen „Achillesfersen-Fächer“: Deutsch für Jungen und Mathematik für Mädchen. Die höhere Sprachbegabung der Mädchen wurde zudem an den Englischnoten der fünften und sechsten Klasse sichtbar. Interessanterweise konnten die Mädchen des Berliner Längsschnitts Medien von der dritten bis zur sechsten Klasse verhältnismäßig deutlicher in Mathematik aufholen (Ende der sechsten Klasse gibt es faktisch fast keinen Notenunterschied mehr), wohingegen die Jungen in Deutsch eher zurückblieben. Ein Befund, der zum einen die Frage nach den Ursachen aufwirft und zum anderen eine gezielte Jungenförderung im Grundschulbereich, zumindest für das Fach Deutsch nahelegen würde. Dieser Gedanke soll später im Zusammenhang möglicher Erklärungsmodelle sowie in den zusammenfassenden Schlussbemerkungen wieder aufgegriffen werden. Neben einer Geschlechtsabhängigkeit der erzielten Schulleistungen, zeigte sich zudem ein auch in den PISA-Studien immer wieder dokumentierter starker Einfluss des sozialen und kulturellen Kapitals auf die Schulleistungen der Kinder (vgl. Baumert et al., 2001; Prenzel et al., 2005; Prenzel et al., 2007; Klieme et al., 2010). Weiterhin zeigte sich neben dem Einfluss des sozialen und kulturellen Kapitals, operationalisiert über das Bildungsniveau sowie den Wohlstand im Elternhaus, ein deutlicher Zusammenhang zwischen dem elterlichen Erziehungsund Unterstützungsverhalten und schulischen Leistungen: Kinder, die über eine geringe elterliche Zuwendung berichteten, hatten schlechtere mittlere Noten als jene, die das elterliche Erziehungs- und Unterstützungsverhalten als mittelmäßig 
einschätzten, welche wiederum schlechter abschnitten als Kinder mit hoher elterlicher Zuwendung.

In einer gemeinsamen Betrachtung des differenziellen Einflusses unterschiedlicher Determinanten schulischer Leistungen, welche sich an das Rahmenmodell schulischer Leistungen aus Abbildung 48 anlehnte, behielten die unterschiedlichen Variablen des sozialen und kulturellen Kapitals, unter Berücksichtigung möglicher Wechselwirkungen, die in den Querschnittskorrelationen ermittelte Erklärungskraft. Darüber hinaus zeigte sich im Einklang mit dem Rahmenmodell schulischer Leistungen (vgl. Abbildung 48) sowie dazu vorliegenden Forschungsbefunden (vgl. Helmke \& Weinert, 1997; Weinert \& Helmke, 1997; Schrader \& Helmke, 2008), dass den kognitiven Merkmalen, gemessen über den IQ, und motivationalen Merkmalen der Kinder, gemessen über die Schuleinstellung, der größte direkte Einfluss zur Erklärung schulischer Leistungen zukommt. Weiterhin entwickelte in vorliegendem Modell insbesondere das Selbstkonzept eigener Schulfähigkeiten, ebenfalls auf Seiten der lern- und leistungsbezogenen Motive und Einstellungen, eine elementare Bedeutung zur Erklärung schulischer Leistungen. Dieser Befund steht wiederum im Einklang mit Befunden anderer Studien zu Determinanten schulischer Leistungen, so zum Beispiel aus dem SCHOLASTIK-Projekt (vgl. Helmke, 1997): Neben der Abhängigkeit vom Bildungsniveau im Elternhaus sowie den kognitiven Fähigkeiten der Kinder sind schulische Leistungen auch in der SCHOLASTIK-Studie korrelativ mit der eigenen Einschätzung schulischer Fähigkeiten verbunden (vgl. Helmke, 1997). Dies bedeutet, dass eine Einschätzung der Kinder, gut in der Schule zu sein, dort schnell lernen zu können und das meiste richtig zu machen sowie insgesamt gut lernen zu können, zu einem nicht zu vernachlässigendem Anteil unabhängig von den kognitiven Fähigkeiten der Kinder mit besseren Schulleistungen einhergeht. Auf der anderen Seite geht eine negative Beurteilung dieser Fähigkeiten unabhängig von den kognitiven Fähigkeiten mit schlechteren Schulleistungen einher. ${ }^{113}$ Das Selbstkonzept eigener Schulfähigkeiten wird seinerseits maßgeblich durch das elterliche Erziehungs- und Unterstützungsverhalten gelenkt. Ergänzt man diesen Befund um die Erkenntnis, dass der Zusammenhang zwischen der Einschätzung eigener Fähigkeiten und schulischen Leistungen über das Grundschulalter immer stabiler wird (vgl. Helmke, 1997), wird deutlich, welch positiven oder negativen Einfluss das elterliche Erziehungs- und Unterstützungsverhalten auch indirekt über diesen Weg auf die Leistungsentwicklung der Kinder entfalten kann. Nicht nur aus diesem Grund kann dieses wohl auch als die wich-

113 Dies soll selbstverständlich nicht bedeuten, dass das Selbstkonzept eigener Schulfähigkeiten nicht von den kognitiven Fähigkeiten der Kinder oder vom Bildungsniveau im Elternhaus beeinflusst wird (vgl. Helmke, 1997). Es gibt nur einen Anteil der davon als unabhängig zu betrachten ist. 
tigste Hintergrundvariable schulischer Leistungen bezeichnet werden. Weitere Auswertungen zur längsschnittlichen Entwicklung des Selbstkonzeptes eigener Schulfähigkeiten unter Berücksichtigung des elterlichen Erziehungs- und Unterstützungsverhaltens wären hier sicherlich gewinnbringend.

Als weiterer interessanter Befund zeigte sich wiederum im Einklang mit dem Rahmenmodell schulischer Leistungen (vgl. Abbildung 48), dass bei gleichzeitiger Kontrolle der Variablen des sozialen und kulturellen Kapitals sowie der wichtigsten Variablen auf Seiten des Schülers, den Variablen der Mediennutzung eine eigene Erklärungskraft schulischer Leistungen zukommt (vgl. Anderson, D. R. et al., 2001; Roe et al., 2001; Schiffer et al., 2002; Ennemoser, 2003; Gentile et al., 2004; Borzekowski \& Robinson, 2005; Hancox et al., 2005; Zimmerman \& Christakis, 2005; Sharif \& Sargent, 2006; Ennemoser \& Schneider, 2007; Johnson et al., 2007; Mößle et al., 2007; Ennemoser \& Schneider, 2009; Hastings, E. C. et al., 2009; Jaruratanasirikul et al., 2009; Skoric et al., 2009; Schittenhelm et al., 2010; Sharif et al., 2010; Baier \& Pfeiffer, 2011). In Übereinstimmung mit früheren Forschungsdaten konnte insbesondere das Spielen gewalthaltiger Computerspiele bzw. das Betrachten gewalthaltiger Filme, ein Nutzerprofil, welches vor allem Jungen kennzeichnete, schlechtere Schulleistungen erklären (vgl. Anderson, D. R. et al., 2001; Schiffer et al., 2002; Ennemoser, 2003; Ennemoser et al., 2003; Gentile et al., 2004; Ennemoser \& Schneider, 2007; Mößle et al., 2007; Ip et al., 2008; Kirkorian et al., 2008; Ennemoser \& Schneider, 2009; Hastings, E. C. et al., 2009; Sharif et al., 2010; Baier \& Pfeiffer, 2011). Teilweise im Gegensatz zur bestehenden Forschungslage hatten die Medienzeiten jedoch keinen direkten Einfluss auf Schulleistungen, bedingten aber wiederum eine deutlich häufigere Nutzung von Gewaltmedieninhalten (vgl. Anderson, D. R. et al., 2001; Razel, 2001; Roe et al., 2001; Subrahmanyam et al., 2001; Schiffer et al., 2002; Ennemoser, 2003; Ennemoser et al., 2003; Gentile et al., 2004; Shin, 2004; Borzekowski \& Robinson, 2005; Hancox et al., 2005; Roberts et al., 2005; Zimmerman \& Christakis, 2005; Chan \& Rabinowitz, 2006; Sharif \& Sargent, 2006; Anand, 2007; Ennemoser \& Schneider, 2007; Johnson et al., 2007; Mößle et al., 2007; Ip et al., 2008; Kirkorian et al., 2008; Ennemoser \& Schneider, 2009; Eow et al., 2009; Hastings, E. C. et al., 2009; Jaruratanasirikul et al., 2009; Skoric et al., 2009; Hofferth, 2010; Schittenhelm et al., 2010; Sharif et al., 2010; Baier \& Pfeiffer, 2011). Somit verloren die Mediennutzungszeiten bei gleichzeitiger Berücksichtigung von Zeiten und genutzten Inhalten sowie der beiden Medien Fernsehen und Computerspiele ihre in den bivariaten Korrelationen beobachtete direkte Erklärungskraft für schulische Leistungen; dieser Befund steht teilweise im Widerspruch zur bestehenden Befundlage. In den Studien, in denen das komplette Medienspektrum berücksichtigt wurde, findet sich stets ein bedeutsamer Einfluss beider Mediennutzungsgrößen, wenngleich, dieser auch je- 
weils für die Nutzungszeiten kleiner ausfällt (Gentile et al., 2004; Mößle et al., 2007; Baier \& Pfeiffer, 2011). Eine erste mögliche Erklärung liegt in der Berücksichtigung einer Vielzahl weiterer Einflussvariablen im vorliegenden Rahmenmodell, die zwar Erklärungskraft der Nutzungszeiten, nicht aber der Gewaltmediennutzung binden, sowie in dem starken Einfluss der Nutzungszeiten auf die Gewaltmediennutzung. Insgesamt können somit gefundene Zusammenhänge oder Nicht-Zusammenhänge anderer Studien möglicherweise darauf zurückzuführen sein, dass entweder nur Fernsehen oder Computerspiele oder ausschließlich Medieninhalte oder Mediennutzungszeiten betrachtet wurden. Zweitens scheint die Berücksichtigung unterschiedlicher Variablen des sozialen und kulturellen Kapitals sowie der Persönlichkeit, die sich nicht nur gegenseitig, sondern auch die Mediennutzung bedingen, essenziell zur Beurteilung des Zusammenhangs zwischen Mediennutzung und Schulleistung. Letztlich ist zur Beschreibung dieses Zusammenhangs die Berücksichtigung des Geschlechts unerlässlich, da dieses das (auch medienspezifische) Mediennutzungsprofil bestimmt. Drittens könnte eine Erklärung der abweichenden Ergebnisse auch darin liegen, dass es früher noch einen höheren Anteil an Kindern gab, welche hohe Mediennutzungszeiten ohne eine auffällige Gewaltmediennutzung hatten, heute beides aber gehäuft gemeinsam auftritt (vgl. $\beta=.33$ ). Letztlich würde eine Interpretation, nur der Konsum gewalthaltiger Medieninhalte wirkt sich negativ auf Schulleistungen aus, aber auch zu kurz greifen, da längsschnittlich, ähnlich wie beim Zusammenhang Mediennutzung und Schulleistung, auch für den Zusammenhang von Nutzungszeiten und Konsum problematischer Medieninhalte von einem gegenseitigen „Aufschaukeln“ ausgegangen werden kann.

Zu 2.) Können Befunde hinsichtlich einer Wirkungsvermutung im Gegensatz zu einer Selektion im Längsschnitt bestätigt werden? Ein erster Eindruck zur Beantwortung dieser Frage und somit zur differenziellen Bedeutung der einzelnen Mediennutzungsvariablen im Zusammenspiel mit den erbrachten Schulleistungen konnte in quer- sowie längsschnittlichen Partialkorrelationen ermittelt werden (kontrolliert für Wohlstand im Elternhaus und kognitive Fähigkeiten): In der längsschnittlichen Betrachtung deuteten die Korrelationen zwischen den Computerspielnutzungszeiten und den Noten tendenziell eher auf eine verstärkende Wirkung der Computerspielnutzungszeiten auf schlechtere Noten hin als auf eine Selektion in dem Sinne, dass Kinder mit schlechten Schulnoten verstärkt exzessiv Computerspiele spielten. Den stärksten Einfluss entwickelten dabei sehr frühe exzessive Nutzungszeiten. Für die Fernsehnutzungszeiten zeigte sich kein vergleichbarer Zusammenhang. Bezüglich der konsumierten Gewaltinhalte ergab sich indes ein eindeutigeres Bild; sowohl für das Computerspielen als auch für den Fernsehkonsum legten die Korrelationen in der längsschnittlichen Betrachtung eine Wechselwirkung zwischen den beiden Medien und den Noten 
nahe, d. h. Kinder mit schlechten Schulnoten spielten verstärkt gewalthaltige Computerspiele und schauten verstärkt gewalthaltige Fernsehprogramme, was wiederum zu schlechteren Noten führte (vgl. Ennemoser, 2003; Ennemoser et al., 2003; Ennemoser \& Schneider, 2007, 2009). Aufgrund der berechneten Korrelationen zeichnete sich also ab, dass bei der Betrachtung des Zusammenhangs von Mediennutzug und Schulleistung weder von einer einseitigen Wirkungsvermutung noch einer Selektion auszugehen ist, es scheint vielmehr ein komplexes Zusammenspiel zu sein. Interessant ist, dass die Mathematiknoten hier eine Ausnahme bilden: Als Ergebnis der berechneten Partialkorrelationen wurden ja nur geringe oder nicht vorhandene Zusammenhänge zwischen den Medienvariablen und den Mathematiknoten berichtet. ${ }^{114}$ Wie kann dieser Befund erklärt werden? Eine mögliche Antwort kommt hier von Spitzer: „Die einfachste Erklärung besteht darin, dass Schüler der Grundschule sich in ihrer Freizeit ohnehin praktisch nicht mit Mathematik beschäftigen, es also nichts durch die Videospiele zu verdrängen gibt. Man liest durchaus gelegentlich in der Freizeit. Und lesen lernt man durch Lesen. [...] Beim Lesen gibt es also was zu verdrängen bei der Mathematik nicht“" (Spitzer, 2010, S. 264). Diese Erklärung fußt allerdings lediglich auf Erkenntnissen zur Zeitverdrängung. Wie obige Auswertungen aber gezeigt haben, kam den Medieninhalten die maßgeblichere Erklärungskraft zu. So könnten diese Befunden beispielsweise auch über die beiden Interferenzhypothesen der Lesebeeinträchtigung oder der Passivität erklärt werden (vgl. Koolstra \& Van der Voort, 1996; Koolstra et al., 1997; Beentjes \& Van der Voort, 1988; Comstock \& Scharrer, 1999; Shin, 2004; Ennemoser \& Schneider, 2007). Möglicherweise ist dieser Befund schlichtweg darauf zurückzuführen, dass Jungen, die ein deutlich medienaffineres Freizeitverhalten mit einer häufigeren Gewaltmediennutzung zeigen als Mädchen, in Mathematik einfach besser sind und Unterschiede dadurch verschleiert werden. Die genaue Erklärung hierfür kann in der vorliegenden Studie leider nicht gegeben werden.

Die berechneten Strukturgleichungsmodelle zum längsschnittlichen Zusammenhang von Mediennutzung und Schulleistung, ein Vorgehen welches erstaunlicherweise bisher lediglich von Ennemoser und Kollegen (vgl. Ennemoser, 2003; Ennemoser \& Schneider, 2007), hier lediglich für die Fernsehnutzungszeiten sowie von Baier und Pfeiffer (2011), hier lediglich für zwei Messzeitpunkte, eingesetzt wurde, beantworteten die Frage nach Wirkung oder Selektion recht eindeutig: Weder noch und beides. So legten die berechneten Strukturglei-

114 Durch die Berücksichtigung der Mathematiknote in allen berechneten Modellen kann von einer konservativen Testung ausgegangen werden, da Zusammenhänge eher abgemildert werden. Dieses Vorgehen wurde aber dennoch gewählt, da mittlere schulische Leistungen abgebildet werden sollten. 
chungsmodelle ${ }^{115}$ bei Betrachtung der Gesamtmodelle eine wechselnde Beeinflussung in zeitlicher Abfolge nahe, wobei mit Ausnahme der Fernsehzeiten der „Start“ jeweils auf Seiten der Medienvariablen zu beobachten war. D. h. beispielsweise, dass Kinder mit schlechteren Noten zunächst verstärkt Fernsehprogramme schauten, was in den späteren Jahren zu schlechteren Noten führte; oder, Kinder mit besseren Noten schauten zunächst weniger fern, was in den späteren Jahren wiederum zu besseren Noten führte. Bei einer regressionsanalytischen Überprüfung zeigte sich zudem, dass bei der Erklärung schulischer Leistungen vor allem der frühkindliche Konsum gewalthaltiger Medieninhalte bedeutsam in vergleichbarer Größenordnung wie das Bildungsniveau im Elternhaus beiträgt. Bei einer Unterscheidung nach Geschlecht zeigte sich jedoch, dass diese Annahmen lediglich für die Jungen gelten. Für die Mädchen sprachen die Befunde eher in Richtung einer einseitigen Selektion in dem Sinne, dass vor allem Mädchen, die in der Schule schlechter abschnitten, in den folgenden Jahren besonders lange Medien konsumierten. Somit zeigte sich auch in diesen Auswertungen die Wichtigkeit einer nach Geschlecht getrennten Betrachtung des zu untersuchenden Zusammenhangs.

Wie kann dieses komplexe Wechselspiel einer gegenseitigen Beeinflussung in zeitlicher Abfolge aber nun erklärt werden? Aufgrund der hier beobachteten $\mathrm{Zu}-$ sammenhänge zwischen Nutzungszeiten und Schulleistungen könnten zunächst Annahmen der Zeitverdrängung zu Grunde gelegt werden (vgl. Neuman, 1988; Comstock \& Scharrer, 1999; Gentile et al., 2004; Cummings \& Vandewater, 2007; Ennemoser \& Schneider, 2007; Mößle et al., 2007). Aber auch Erklärungshypothesen, die auf die konsumierten Inhalte fokussieren, haben aufgrund der Befunde zum Zusammenhang von Medieninhalten und Schulleistung ihre Berechtigung. Insgesamt scheinen die Befunde aber sehr gut zu der von Anderson und Kollegen beschriebenen Spirale zu passen (vgl. Comstock \& Scharrer, 1999; Anderson, D. R. et al., 2001), wobei der Weg meines Erachtens nicht zwangsläufig über ein auffälliges Verhalten und Interaktionsprozesse zwischen Lehrkräften und Schülerinnen und Schülern gehen muss. Hypothesen, die lern- und leistungsbezogenen Motive und Einstellungen in den Vordergrund stellen, können bei einer Entstehung dieser Spirale auch herangezogen werden (vgl. Koolstra \& Van der Voort, 1996; Koolstra et al., 1997; Beentjes \& Van der Voort, 1988; Comstock \& Scharrer, 1999; Shin, 2004; Ennemoser \& Schneider, 2007). So hing eine negative Schuleinstellung mit einer erhöhten Mediennutzung zusammen und wirkte sich direkt auf schulische Leistungen aus. Auch die Fähigkeit zum Bedürfnisaufschub, welche zum Beispiel in Computerspielen, in denen man schnell reagieren muss und schnell zum Erfolg kommen muss, wenig gefördert wird,

115 Die hohen Stabilitäten der Schulnoten ließen dabei insgesamt wenig Erklärungsspielraum für mögliche Unterschiede. 
könnte hier von Bedeutung sein. Zudem besteht ein elementarer Unterschied zwischen Inhalten und Darbietungsform im Unterricht und in den Medien, welcher sich ebenso motivational auswirken könnte. Diese Deutung könnte man auch als Lernmodushypothese bezeichnen (vgl. Mößle, Bleckmann, Rehbein \& Pfeiffer, 2011). Diese Hypothese wird gegenüber einer reinen Zeitverdrängungswirkung des Medienkonsums durch die vorliegenden Daten insofern eher gestützt als der reine Nutzungszeiteneffekt gegenüber einem Inhaltseffekt zurücktritt. Letztlich muss insgesamt ein Zusammenspiel der unterschiedlichen Erklärungsannahmen angenommen werden, in dem neben einer zeitlichen Verdrängung auch motivationale Prozesse sowie Beeinträchtigungen der kognitiven Leistungsfähigkeit ihre Rolle spielen. Ein Überprüfung einzelner Wirkannahmen ist jedoch nicht nur in dieser Studie äußert schwierig und zum Teil unmöglich. Abgesehen von dieser fehlenden Spezifizierung zeigte sich in dieser Studie aber eines recht deutlich: Eine zeitlich exzessive sowie inhaltlich problematische Mediennutzung hat neben anderen Variablen ein eigenes Wirkpotential auf schulische Leistungen, wobei Jungen durch ihren im Vergleich zu den Mädchen „exzessiveren“ Medienkonsum stärker beeinträchtigt zu sein scheinen. 